\title{
Index to Geophysical
}

Abstracts 180-183

1960

By JAMES W. CLARKE, DOROTHY B. VITALIANO, VIRGINIA S. NEUSCHEL, and others

3 E OLOGICAL S URVEY B U L L E T I N $1116-$ E

Abstracts of current literature

bertaining to the physics of

the solid earth and to

geophysical exploration

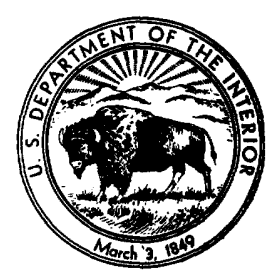




\title{
UNITED STATES DEPARTMENT OF THE INTERIOR
}

STEWART L. UDALL, Secretary

\author{
GEOLOGICAL SURVEY
}

Thomas B. Nolan, Director 
By James W. Clarke and others

AUTHOR INDEX

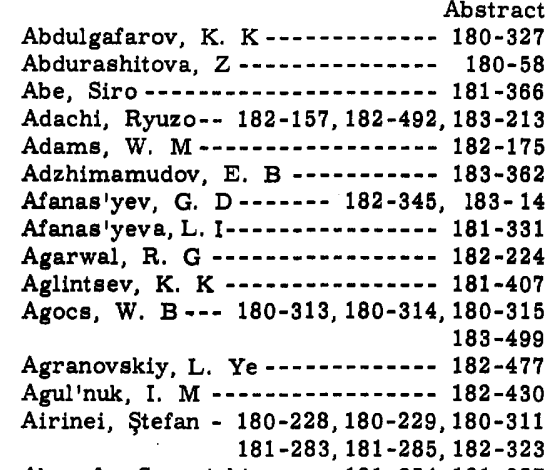

Akasofu, Syun-ichi -..- 181-354, 181-357 $183-435$

Aki, Keiiti - -.- 180-55, 181-111, 182-117 Akimoto, Syun-iti-....- 180-271, 180-274 $180-279,180-280,180-290$ $181-391,183-451$

Aksel'rod, S. M Aksenovich, G. I Alaska University Aldrich, L. T - 181 -35, 183-5 Alekseyev, A. M Alekseyev, F. A------ 183-516, 183-525 Alekseyev, V. V-181-143 Alekseyeva, K. N-_-_-_-_.-. 183-75 Alexander, Corrinne -..-.-.-- 182-29 Alexander, J. B - - 180-314, 180-315 Alexander, N. S-_. $182-386$ Alger, R. P-182-203 Aliev, T. M-D 183-266 Alizade, G. A Alldredge, L. R - ......- 183-420 Allen, C. R Allingham, J. W-...... 183-477, 183-485 Al'pin, L. M - ........ 183-229, 183-260 Ambraseys, N. N--180-68, 181-94, 181-150 Amirkhanov, Kh. I-...... 182-10 Ananyan, A. L Anderle, R. J -....... 183-315 Anders, Edward --- 182-66, 183-63, 183-73 Andersen, S. T-. 182-41 Anderson, D -....... 181-211 Anderson, D. L . . . . . . . Anderson, L. A -.... 183-267 Anderson, R. H -..... 180-334 Ando, Takeshi-............. $182-545$ Andreasen, G. E -183-465, 183-486, 183-492 Andres, Jakob-... 182-503
Abstract

Andreyev, B. A -

Andreyev, T. A -

Andreyeva, R. I - . - 181-221, 183-500

Angenheister, Gustav -.....-.-180-268

Angona, F. A - ...... 181-173

Anstey, N. A -

Aok1, Harumi - 180-368

Aquilina, Carmelo-_....... 181-226

Araki, T -......... 180-265

Arkhangel'skaya, V. M -._... 181-135

Arkhangel'skiy, V. T -...- 180-65, 181-442

Armatrong, F. E - .

Arnold, Kurt -- 180-181, 182-248, 182-251

$182-283,182-288,183-313$

Arutyunyan, E. A -...- 182-427, 183-456

Asano, Shuzo-..--180-100, 180-101

Aslanyan, A. T -

Assiter, E. J -

Aswathanarayana, U -....... 181-51

Atchison, T. C - 182-168

Aten, A. H. W - 182-46

Atrashenok, L. Ya -.... 183-40, 183-46

Ault, R. K -

Ault, W. U -

Avdzeyko, G. V -

Avsyuk, Yu. N -... 182-507

Azhgirey, G. D - 183-320

Baadsgaard, H -

Babenkov, Ye. F - 183-392

Bachelet, F -

Bacioiu, Traian -

Bagdoyev, A. G - 183-183

Baile;, V. A -

Bak, M. A - $181-62$

Baker, George -...... 181-71, 183-83

Balabushevich, I, A - -

Balakina, L. M -.... 180-47, 181-320

Balakrishna, S - . _...

Balashov, D. B - 181-168

Balata, P - 182-395

Balavadze, B. K -..... 181-307

Baldwin, Brewster -..... 182-410

Balsley, J. R. ,Jr -181-378, 183-489, 183-491

Bancroft, A. M -.. $182-295$

Band, William -... 181-163

Banno, Noboru -.................. 180-265

Banville, J. H -... 183-564

Baranov, V. I -

$181-64,183-293,183-540$

Baranovskaya, N. V ....... 181-8

Baranovskiy, V. I - 180-332

Barker, H -...... 182-21 
Abstract

$181-34$

Barr, K. G - 183-110

Barsukov, O. M - - 181-83, 181-362

Barta, György ------181-349, 182-307

Bartnitskiy, Ye. N-... 182-10

Bascom, Willard -..--181-309, 181-310

Bateman, J. D -.... 182-223

Bates, R. G - 183-530

Båth, Markus -$182-129,182-354$

Baule, H -

Beard, D. B ----180-28, 183-413, 183-433

Beck, A. E -

Becker, Alfred-... 180-157

Bednár̆ová-Nováková, Bohumila -- 182-392

Begemann, Friedrich -......-- 181-323

Bĕhounek, Rudolf-_...- 181-398

Behrendt, J. C - 181-293

Bellair, Pierre -.... 181-494

Belluigi, Arnaldo--.-.-- 180-103, 181 -177

Belotelov, V. L - .

Belousov, V. V- 181-243, 183-318, 183-346

Bélteky, Lajos -....... 180-159

Bemmelen, R. W. van --..... 183-329

Beneo, Enzo -...-182-313

Benioff, Hugo -...-.-180-61, 181-140 $181-141,181-370$

Ben-Menahem, Ari-_..-- 181-121, 183-136

Bennett, G. D-_- 183-254

Benoit, Réné-182-387

Beránek, Břetislav------ 181-447, 181-475 $182-291,182-320$

Berdichevskiy, M. N-_... 181-193

Bereza, G. V - 183-166

Berg, E -

Berg, J. W. , Jr -- 180-97, 183-204, $183-395$

Bergey, W. R - 180-125

Berkman, R. Ya-.-... 183-472

Bernard, Pierre-1-181-146

Bernshteyn, V. A--.--- 181-314, 182-432

Bernstein, F -......-180-140

Berry, J, E - . - 180-373, 181-452

Berzon, I. S - - 180-370, 182-127

Besairie, Henri …...... 183-22, 183-23

Bessoles, B-.... 183-24

Bhimasankaram, V. L.S - - 182-405

Bidgood, D. E. T - 180-284

Bien, G. S - 182-31

Biggs, W. P _..... 182-202

Bigotte, G - $180-345$

Bike, P. B-.. 183-302

Billings, M. P -

Biq, Chingchang - - $182-273,183-331$

Birch, Francis - ...... 182-338, 183-198

Birkenmajer, K -....... 183-343

Bisby, H -... 181-434

Bishopp, D. W - $182-262$

Bisztricsány, Ede -.-.-- 181-104, 182-107

Black, M. C - 183-180

Blackadar, R. G-_. 182-39

Blackwell, M. J . ........ 183-289

Bland, D. R -

Blankov, Ye. B-....... 181-423, 181-424

Blankova, T. N-_- $181-423$

Blanpied, B. W-.... 182-217, 183-277

Bless, R. C $\ldots \ldots 181-358$

Blundell, D. J-180-292
Blyumentsev, A, M-_.-Asstract Boaga, Giovanni -180-177, 182-249, 182-253 Bobier, Claude -............. 181-387

Bochkarev, V. V -......... 181-407

Bock, R -

Boerboom, A. J. H -

Bois, P -

Boku, T - . 180-279

Boldizsár, Tibor--_---182-333, 182-335

Boletin Sismológico del Servício

Geológico Nacional de E1

Salvador - - 183-109

Bomford, G -....... 181-233

Bondarenko, A. T - - - 182-214, 183-274

Bonelli Rubio, J. M-_-_- 182-123

Bonilla, M. G - 181-88

Bonini, W. E - $181-469$

Boniwell, J. B -

Bonnet, J - . . 183-246

Bordovskiy, V. P - $181-190$

Borodachev, N. M - 180-82

Boronin, V. P-_. 183-381

Bortfeld, Reinhard -...-. 182-486, 183-179

Bossy, L -.... 181-79

Bot, A. C. W. C- $182-46$

Botezatu, Radu-_-181-284, 181-286

Botneba, T. A-181-21

Bott, M. H. P -

Bouchon, R -

Boushka, Ya - .

Bower, M. E -....- 183-476, 183-498

Bowie, S. H. U -

Braaten, N. F-2 180-194

Brace, W. F-n-181-481, 183-579 Bradley, J. S -

Brandt, S. B - $182-10$

Brant, A. A-182-184

Braun, T. H - 182-236

Bray, E. E -

Brazee, R. J - 180-33, 183-97

Breyer, F - 183-282

Briggs, M. H -.... $183-50$

Brock, B. B -

Brod, I. O -... $181-216$

Brod, R. J -..... 182-424

Broding, R. A -..... 182-496, 183-560

Brodskaya, S. Yu _.

Broecker, W. S - -

$183-403,183-404,183-506$

Bromery, R. W- $-183-478,183-479,183-480$ $183-481,183-483,183-489,183-491$

Brossard, Leo -

Brower, D -

Brown, Harrison - 182-61

Brown, P. D-180-386, 180-390

Brown, R. E- 183-309

Brownell, G. M - 180-344

Brož, Václav-..... 181-440

Brune, . J. N -....-181-118, 183-156

Bryant, H. L -

Bryunelli, B. Ye --180-29, 181-143, 183-421

Bubnov, S. N. von - 180-200

Bucha, Václav --181-350, 182-380, 182-381

Buddington, A. F _...... 181-378

Buffet, A - .

Bukhteyev, V. G ........... 181.404 
Abstract

Bulashevich, Yu. P-_- 181-408

Bulin, N. K - 183-153

Bull, C -

Bullard, F. M- 183-593

Bullen, K. E- 181-468

Bullwinkel, H. J-_._._. 182-36

Bülow, Kurd von -..... 183-51

Bune, V. I -

Bureau of Mineral Resources,

Geology and Geophysics - . - - 183-288

Burkard, Otto-..-181-365

Burkard, R. K -

Burke, K. C -

Burke, W. H. , Jr -

Burling, R. Q -

Burman, S. D -

Burri, J. P - $182-190$

Burša, Milan ---_-.-- 182-252, 182-303

Burtmar, M. S _..... 181-219

Burton, R. P -

Buryakovskiy, L. A --.-- 180-165, 183-262

Butakov, G. S -

Butkovich, T. R -..... 180-208, 182-277

$182-517,182-518,182-519$

Buwalda, Phyllis -... 183-88 Bycroft, G. N Byerly, Perry-_-_._-_ 181-85, 181-110 Byerly, P. E Bykovskaya, E. V Byus, Ye. I -

Cagniard, Louis Cahen, Lucien -............. 183-21

Cailleux, André -............ 182-280

Cameron, A. G. W-

Cameron, R. L

Campbell, O. E -

Campbell, W. G-........ 182-225

Campbell, W. H -181-359, 181-360, 182-389

Canada Geological Survey -183-493, 183-494

$183-495,183-496,183-497$

Capron, P. C - $182-16$

Caputo, Michele -

Carder, D. S - .

Carey, S. W -

Carissimo, L - -

Carpenter, E. W -.... 183-180

Case, J. E- E 183-487

Catanzaro, E. J - $182-4$

Cavallero, Carmelo -..._. 181-498

Cawley, A -

Cecchini, André -

Chapman, Sydney - - - 180-174, 183-444

Chauris, Louis -............ 182-42

Chauveau, Jean -..-182-163, 183-539

Cheng-Yi, Fu -....... 181-231

Cherdyntsev, V. V----- 180-327, 180-328

$182-450,183-410,183-508$

Chernyshev, N. I-- 180-144

Cherry, R. D - 183-78

Chetayev, D. N-181-181, 182-180

Chikvaidze, B. G-.... 182-13

Chin, Chen - $180-199$

Chinburg, D. L ....... 182-400

Chinnery, M. A $\ldots \ldots \ldots 183-137$

Chirvinskaya, M. V -

Chisholm, E.

Chombart, L, G -

Choubert, G -... 183-25

Chovitz, Bernard -..... 180-187

Chudoba, Vratislav -........ 182-315

Chujo, Junsuke - $181-438$

Chukin, V. T _..... 183-257

Chupakhin, M. S - 180-251

Cipa, Walter -... 180-310

Claire, C. N-183-337

Clark, A. R -...... 180-125

Clark, D. B -

Clausing, D. P - 182-512

Cleary, J -.

Closs, Hans -

Cloud, W. K - - - $180-95,181-90,183-97$

Cobb, Howard -

Cobb, J. C -... 181-28

Cohen, A. J-- 183-84

Cohen, C. J-183-315

Cole, K. D - 182-374

Coleman, P. J. , Jr -

Collin, C. R -.... 180-149

Collins, Francis-......... 182-150

Collinson, D. W -

Combe, R -...... 183-246

Compston, W----180-250, 182-55, 182-369

Conforto, A. M -

Conley, J. M -

Conn, H. K - 180-300

Conselman, F. B -

Cook, A. H - - - 180-176, 180-179, 182-287

Cook, J. C-_..... 182-213

Cook, K. L -

Cook, M. A - 183-6

Coppens, Réné - - 183-509

Cormier, R. F ----181-24, 181-38, 181-73

Corpacius, Alexander - 183-316

Cotecchia, V -.... 180-160

Coulter, H. W-183-18

Couwenberg, G-........... 182-46

Cowling, T. G - 183-418

Cox, Allan -

Crane, H. R - $182-22$

Crary, A. P ----181-293, 182-40, 183-572

Crawford, J. M -

Creer, K. M -

Crenn, Yvonne -

Crevecoeur, E. H -...... 182-16

Crocket, J-n 181-10

Cromie, W. J-n 181-488

Crowell, J. C -

Crozier, W. D - 183-57

Culver, R. C - 180-172

Cummins, D. O -

Currier, L. W -

Curtis, G. H

Dachille, Frank -... 181-313 da Costa, J. A -

D'Agostino, O -

Dakhnov, V. N --182-206, 182-208, 183-255

Daly, J - .

Dance, D. F - $182-46$

Danilevich, S. I -..... 180-5, 180-6, 183-9

$183-34,183-35,183-38$ 
Abstract

Danjon, André -

Dansgaard, Willi -...- 181-327, 183-406

David, E -

Davidson, C. F-...-180-2, 181-406, 183-4

Davidson, R. J -

Davies, R. M -. 180-83

Davis, G. L-182-37

Davis, T. N-_-_-_-_ 183-104, 183-108

Davis, W. E -... 183-364

Davydov, B. I -

Davydova, N. I -

de Anda, L. F -

de Bremaecker, J. C1 ........ 181-98

DeChow, E-... 181-330

Deevey, E. S -

DeFelice, J - .

Delaney, C. F. G - - 181-16, 182-455

Delaplanche, J -........... 183-541

de Lapparent, C -..._. 183-573

Delevaux, M. H -... 183-16

Delibrias, Georgette -........ 180-20

Den Geofysiske Kommisjon -...-. 180-175

Den, Nozomu--... 180-100

Denisov, Ye. P -

Dennison, A. T - 181-209, 181-445, 183-537

Deresiewicz, H -.... 183-178

Derksen, George -..... 183-528

Desio, A - . .

Dessler, A. J-- 180-257, 181-337

$181-356,183-440$

Deutsch, E. R - 180-389

Deutsch, Sarah --18--181-19, 182-42

Devirts, A. L -.... 181-17

de Vries, Hessel -...-. 182-38, 182-41

De Vuyst, A _........ 181-79

D'Hoeraene, $J$ - - 183-549

Dianov-Klokov, V. I -_.

Diceglie, Stefano--.-.-- 180-160, 182-191

Dickey, D. D - 183-200

Dietz, R. S -

Di Filippo, Domenico --...-.- 181-106 Diment, W. H - - 183-98, 183-199, 183-372 Dimitrov, L. V - 180-223 Dix, S. H - 180-86 Dizioğlu, M. Y - 182-189 Dmitriyev, V. I _..... 181-191 Dobkina, E. I Dobrin, M. B Doell, R. R - - - 182-406, 183-457 Dohr, Gerhard Dolan, W. M - - 183-395 Doležal, Jindřich - - - 182-316, $182-318$ Dolginov, S. Sh Dolina, L. P Dolitskiy, V. A - - 180-145 Doll, H. G - - 182-200, 183-303, 183-304 Dominici, P - -...- 183-296 Donabedov, A. T - 183-358 Dontsova, Ye. I --.-. 181-60, 183-405 Dooley, J. C - ........- 180-233, 180-254 Dorman, James -...- 181-130, 183-189 Dorofeyev, B. V -..... 183-237, 183-240 Doty, W. E. N - 180-379 Doubourdieu, Georges -........ 181-97 Doyle, H. A -..... 180-102 Drake, C. L . 180-202
Abstract Dreimanis, Aleksis --_-_---- 182-38 Droste, Zofia -........ 180-53, 181-114 Du Bois, P. M Dubrovskiy, V. G - 180-51 Duda, S. J - 183-181 Due Rojo, Antonio -..-_ 182-437 Duffus, H. J Dumanoir, J. L _... 182-200 Dumitru, Stelian -..-181-286 Duncan, R. A Dunlap, H. F Dupouy, Georges -........ 182-401 Dupuy, Mlle -...... 180-31 Durand, G. L Duvall, W. I -....... 182-168 D'yachkov, N. P-_._-_ 181-227, 183-475 D'yakonov, B. P - - 180-111, 181-178 $183-225,183-226$

D'yakonova, M. I --_---- 181-69, 183-58 Dyck, W -....... 182-25 Dymáček, František -........ 181-440 Dzhafarov, Kh. D -180-109, 183-238, 183-380

Eaton, G. P Eaton, J. P _..._-_ 180-35, 183-588 Eberhardt, Peter--_-_--- 182-71,183-71 Ebert, K. H Echagaray, F. A Eckelmann, W. R - 183-506

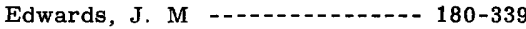
Egyed, László---- $181-259,182-58,182-59$ $182-267,182-339,183-325,183-365$ Ehara, Shingo -....... 182-272, 183-332 Ehmann, W. D -...... 183-82 Eife, K. H - $180-342$ Ekren, E. B Electronics -..... 182-332 Elkins, Paul Ellis, G. R. A - 181-367 Ellison, M. A - A. E1 Nadi, A. F -...- 183-518 Emery, K. O --------- 182-523, 183-489 Emiliani, Cesare Enenshteyn, B. S-_-_-_ 181-195 Enescu, Dumitru -.......... 181-125 Engineering and Mining

Journal - . . . . . 182-239

Engstrand, L. G - 182-30

Erfle, M. E - 180-293

Eshelman, V. R _...... 182-62

Everdingen, R. O. van --.--- 180-285 Everingham, I. B -180-102, 180-233, 182-326 Evernden, J. F -... 183-12 Evrard, Pierre -...-- 182-227 Ewing, J. I -....... 180-243, 183-583 Ewing, Maurice------- 180-191, 180-202 $180-217,181-124,181-130$ $181-490,182-159,182-160$ $182-174,182-347,183-403$

$\mathrm{Ez}, \mathrm{V} . \mathrm{V}-\ldots 181-246$

Faessler, C. W -

Fairbairn, H. W -.- 181-24, 181-26, 181-38 $181-39,181-41,181-73,182-36$

Fairbridge, R. W-D 182-264 Fajklewicz, Zbigniew -.......-- 182-296 
Abstract

Farner, D. M - 180-329

Farquhar, R. M -... 181-7

Fatt, I-_. $181-162$

Faul, Henry ------180-13, 182-1, 182-43

Faure, G - $181-26$

Faust, L. Y - 182-484

Faytel'son, A. Sh-183-369

Fechtig, H -.... 181-57

Fedorov, Ye. P -

$180-80,181-147$

Fedorova, V. A -

Fedosenko, N. Ye_....... 181-144

Fedynskiy, V. V-_._. 182-241

Ferchev, M. D -.... 183-131

Ferenczi, Istvan-182-268

Fergusson, G. J - - 181-324, 182-449

Fesenko, Yu. V -

Fesenkov, V. G -.... 183-55

Filippov, M. S ---- 180-5, 183-34, 183-35

Filippov, Ye. M--

Filonov, V. A - 183-516

Finsterwalder, Richard -...-- 180-213

Fiorentini-Potenza, M -..-1-182-445

Fireman, E. L -.....- 181-61, 183-62

Firsov, L. V - -

Fischer, Georg -....... 182-340

Fischer, Irene $180-187,180-188,182-250$

Fish, R. A -... 183-73

Fisher, D. E -...... 182-73, 183-65

Fisher, N. H -

Fisher, R. L -... 181-489

Flanagan, F. J-183-522

Flanders, P. L -.... 182-175

Fleming, H. W -... 181-394

Flinn, E. A

Florenskiy, K. P - 181-58

Florensov, N. A - -

Fogel', A. A -

Fogelson, D. E -

Fokin, A. F - $182-181$

Folinsbee, R. E - 183-1

Fomina, V. I -..... 183-242

Förtsch, Otto -..-- 181-474

Fotiadi, E. E -

Fowler, W. A - 183-69

Fowles, G. R - 182-153

Francis, W. E -

Franke, R -

Frantz, J. C -

Fraser, G. D - 183-587

Fremd, V. M -.......... 183-173

Fridman, Sh. D -

Friedman, Irving -...... 181-323, 182-527

$183-81,183-402$

Frischknecht, F. C -..-- 180-128, 183-222

Fristrup, Børge - C 182-278

Fritsch, Volker -... 182-187

Frölich, Friedrich -

Frolova, A. V - 182-128

Frosch, Alex -....... 183-461

Frost, H. H - $181-464$

Frye, J. C - 181-30

Fujiwara, Chuichi -......... 183-263

Fukushima, Naoshi -....... 182-377

Fuller, M. D -........ 182-404

Furuya, Shigemasa -... 180-384, 183-249
Abstract

Gaibar-Puertas, Constantino ----- 180-309 Gall, Ruth -... 183-426 Gallagher, P. B - $182-62$ Gal'perin, Ye. I -181-462, 182-128, 182-356 Galushko, P. Ya Galuzo, Yu. V -........ $181 \div 425$ Gane, P. G - 182-353 Gangloff, A. M - 180-149 Gantar, C - 181-267 Gardner, J. K - 183-101 Garland, G. D -- 182-198, 182-237, 183-476 Garrison, Robert -..... 181-411 Gartlein, C. W - 181-358 Gasanenko, L. B -...-- 181-183, 181-184 Gast, P. W - . $181-55,181-316,182-4$ Gastil, Gordon -..--180-3, 183-324 Gavelin, Sven Gavrilov, L. I Gavrilova, L. K - 183-37 Gayskiy, V. N Geier, S - - 181-185 Geiss, Johannes -...-.- 180-204, 183-71 Gel'fand, I. S --_._-_ 183-227, 183-228 Gel'man, O. Ya _..... 182-14 Gentner, W - 181-57, 182-75 Geologiya Nefti _ Geophysical Prospecting -...-- 183-542 Geophysics Research

Directorate - 183-294 Gerard, Robert -... 183-403 Gerecke, Friedrich - - - 183-208 Gerling, E. K ...... 180-10, 181-331 $183-13,183-31$ Geyl, W. F Ghazarian, G. B - $182-192$ Gheith, M. A -... 181-23 Gibson, M. R - 181-431 Giffin, C. E Gigout, Marcel -..... 181-43 Gil'bershteyn, P. G -.... 182-165 Gilbert, C -. 182-281 Gilbert, Freeman -...-181-112, 181-119 $181-155,182-137,183-150$

Gilbert, R. L. G - 181-266 Giletti, B. J Gill, P. S Gilman, Ralph -.-167 Gilvarry, J. J - 181-296 Gintzburg, M. A - 183-232 Girdler, R, W -..-- 180-283, 183-469 Gladkiy, K. V Glangeaud, Louis Glebovskiy, Yu. S -....... 181-402 Gledhill, T. R Glivenko, Ye. V - . - 181-133, 182-122 Gloyna, E. F Glukhov, I. G - 182-124 Glyuzman, A. M-183-233, 183-387, 183-388 Gnilovskoy, V, G-_. 181-68 Godin, Yu. N -- 181-215, 182-241, 183-576 Godwin, H Goebel, K -...-. 181-65, 183-64, 183-74 Goedicke, T. R . Gokhshtein, Ya. P ...... 181-6, 182-456 Goldberg, E, D ........... 181-329 Gol'dgefter, V. I - 182-196 
Abstract

Goldich, S. S - $182-56$

Goldstein, M. I -

Golebchina, M. N - 180-252

Golenetskiy, S. I - . . 183-135

Goles, G. G - 183-73

Golovina, I. F - 183-596

Gol'tsman, F. M -

$182-479,182-480$

Gonfiantini, R - 180-249

Gorin, V. A - 181-96

Gorshkov, G. P _. 181-101

Gorshkov, G. S - 180-406

Gorshkov, G. V -...-- 181-414, 183-507

Gorung, M. B - 183-111

Goryachev, A. V - 182-99

Gorzhevskiy, D. I -

Gosselink, J. G - 180-205

Gottfried, David -...-180-16, 180-17

Gottis, Maurice -

Gough, D. I -

Gowen, K -... 181-10

Grablenskiy, V. N-_... 181-407

Grabovskiy, M. A - $183-458$

Graebner, R. J - 180-363

Gralenski, L. J - $182-23$

Grant, F, S -

Grantz, Arthur -...--- 183-490, 183-492

Grau, Gerard -...---182-163, 183-539

Grave, N. A -... 183-352

Gray, Carlyle-18 - 183-482

Gray, Helen -.... 180-362

Grechukhin, V. V - 180-146

Green, Jack -... 183-89

Green, M. I - $181-356$

Green, R -.... 181-379

Greene, G. W - 183-334

Gregory, J. N - 183-563

Gretener, P. E. F - 183-559

Griffin, J. B - $182-22$

Griffiths, T. M - 182-276

Griggs, D. T - 182-258

Grigor'yev, I. G - 182-14

Grimbert, Arnold -.... 180-149

Grinda, Louis - $181-123$

Grine, D. R -

Grinenko, V. A -

Grodzenskiy, V. A _...... 181-441

Groeneveld, D -

Grosse, Siegfried - - $181-280$

Gruntorad, Yan -....-- 181-188, 181-194

Grushinskiy, N. P -

Gubin, I. Ye - 180-41

Guillou, R. B -

Gurevich, B. N - 181-455

Gurvich, I. G -..... 182-11

Gurvich, I. I - 182-165

Gushenko, I. I -

Gutdeutsch, R - $182-493$

Gutenberg, Beno -180-56, 180-320, 181-122

$181-131,181-303,182-106,183-149$

Gzovskiy, M. V -..... 181-249, 182-89

$182-96,183-580$

Hada, S -...- 183-555

Haeberle, F. R -

Haites, T. B - 183-338
Abstract

Halbouty, M. T -

Haldemann, E. G -..... 183-3

Hale, F. H -.... 181-434

Halenka, Jaroslav -......... 182-391

Hales, A. L - 180-227, 182-352

Haman, B. O -... 182-193

Hamer, A. N - ....... 183-412

Hamilton, E -

Hand, J. E -

Handin, John - $182-508$

Hansen, R. T - 181-361

Hardin, G. C. , Jr

Hardy, H. R. , Jr- 182-511

Hardy, H. W -

Harkrider, D. G _..... 180-243

Harland, W. B - 180-284

Harpum, J. R -

Harris, J. L - . 180-360, 180-395

Harris, M. A - 183-39

Harris, P. G - 182-359

Harrison, J. C - 182-301

Harrison, J. V --.-.-- 180-224, 182-266

Harrison, W -..... 182-32

Hart, S. R - 181-34

Hasegawa, M - 181-353

Hatai, Kotora -

Hatherton, T -

Hattori, Yasumasa - . 183-556

Haubrich, Richard, Jr -..... 180-73

Haubrich, R. A - 181-293

Havemann, Hans ........-183-91

Hawkins, G. S -_-_-_ 181-72, 182-76

Hayakawa, Masami -...- 180-235, 182-164

Hayasaka, Shozo -_...-_-_-_ 183-345

Hayase, Ichikazu -180-326, 182-446, 182-448

Haye, E. F - 181-261

Hayter, P. J. D - $182-526$

Heacock, J. G - 182-458

Healey, D. L - 183-372

Healy, James -

Healy, J. H -... 183-191

Hédervári, Péter -.... 182-80

Hedström, E. H - . . . - 180-113, 183-223

Heezen, B. C -....- 181-490, 183-403

Heirtzler, J. R -

Heiskanen, W. A -181-232, 182-312, 183-310

Heisler, L. H -

Hellbardt, Gunter-1...-180-277

Henderson, J. R. , Jr-_-_-_- $183-479$

Henderson, R. G - 182-417

Hennion, J. F - 180-243

Heppner, J. P - 181-340

Hernegger, F - $183-74$

Herr, W - $182-70$

Herrin, Eugene -...-. 181-137, 183-155

Hervas Burgos, Pablo -...-..- 182-155

Herzenberg, A - .

Herzog, L. F - 181-9, 181-73

Herzog, L. F., II -.... 182-5

Hess, D. C - 182-71

Hess, H. H -

Hess, W. H - 183-519

Hessler, V. P - - - 182-81, 183-93, 183-94

Hieblot, James -

Hiern, M. N - 182-473

Hill, G. E - 183-442 
Abstract

Hill, M. N -

Hilty, R. E -

Hines, C. O -..... 180-258, 181-355

Hintenberger, $\mathrm{H}$

Hinze, W. J - 181-264

Hiraga, Shiro --_.... 182-539, 182-540

Hirono, Takuzo -

Hirshman, Julius - - - - 183-422, 183-583

Hirvonen, R. A - 183-311

Hobson, G. D -

Hodgson, J. H -

Hoering, T. C -

Hoffman, J. H -.. 180-23, 182-56, 182-72

Hoffmeister, W -...... $182-70$

Hofmann, R. B - 183-98

Hogan, T. K - 180-102

Hohl, Rudolf -...- 183-379

Holgate, M. M -

Hollinderbäumer, J - - $182-439$

Holmes, Arthur -..... 180-1, 183-21

Homilius, Joachim -

Homma, Ichiro -- - $180-168,183-249$

Honda, Hirokichi - .

Honkasalo, Tauno ---.- 181-279, 183-341

Hopkins, W. B - $182-85$

Horai, K -

Horikawa, Yoshio -180-168, 180-355, 180-357

Horr, C. A -...- 182-370

Horvath, Josef -..... 180-317, 180-319

$180-135,181-401$

Hosono, Takeo -_..-- 180-358, 180-359

Hospers, J - - 182-311, 183-377

Housner, G. W -... 182-86

Howard, Hildegarde -......- 181-31

Howell, B. F. Jr -

Howell, L. G -... 183-461

Hoyle, F -

Hoylman, H. W - 180-293

Hubbs, C. L - 182-31

Hudson, D. E -

Hunkins, Kenneth -

Hunt, C. B - 183-334

Hunter, W -

Hurley, P. M -

$181-12,181-22,181-24,181-25$

$181-26,181-27,181-32,181-34$

$181-37,181-38,181-42,181-44$

$181-52,181-332,182-36,182-46$

Hurtgen, H -

Hurwitz, L -..... 181-341

Hussey, K. M - 183-18

Hutton, Rosemary - 182-384

Ibrmajer, Jaroslav -

$182-318,182-319$

Iida, Kumizi - 180-368

Illies, Henning -

Imai, Shigeyoshi-_-_-_-_-5 183-578

Inagaki, M -

Inami, Kazui -... 180-384

Ingraham, R. L -

Ingram, R. E - 183-160

International Geophysical Year

Bulletin -........ 180-185, 180-399

$181-473,182-226,182-350$

Iosif, T - $181-107$
Abstract

Irving $\mathrm{E}-\ldots-180-281,181$-384, 181-392

Ishibashi, K Ishida, Tamotsu-...-- 180-93, 180-94

Ishigaki, Akira -.... $181-466$

Ishikawa, S - . - 180-263

Ishikawa, Yoshikazu ----180-271, 180-272

$180-273,180-274$

Islamov, S. Sh -

Isräel, Hans - 180-330

Itenberg, S. S -

$183-272,183-306$

Ito, Haruaki - $182-409$

Ito, Ichiro - . . . . $182-167$

Ivakin, B. N - $181-172$

Ivankin, P. F - $181-292$

Ivanova, K. S _. 180-6

Ivanova, T. G - 182-489

Iwai, Takehiko - . .

Iwasaki, Shoji - - - - 180-348, 180-349

$180-350,180-353,180-358$

Iyer, H. M -

Izaki, Akira - 183-557

Izergin, A. M - 183-95

Izokh, E. P - $182-52$

Izotov, A. A

Jackson, J. E -

Jackson, W. H -

Jacobs, J. A -

$181-372,183-375,183-436$

Jaeger, J. C - - 180-236, 180-398, 181-482 Jaffe, H. W Jäger, Emilie --......- 180-13, 182-43 Janoschek, R. H Janssens, P - .

Jeffery, P. M - -

Jeffreys, Harold -...- 180-76, 180-77

$182-341,182-513$

Jelen, Miroslav -_. $182-426$

Jellinek, H. H. G -

Jenkins, A. W. Jr-_. 181-377

Jenness, S. E ...... 180-276

Jensen, K. D - $181-444$

Jeou-jang, Jaw - 181 -231

Jobert, Georges - - _... 182-147, 182-304

$182-329,182-330,183-141$

Jobert, Nelly _... _. _ 182-130, 183-157

Joesting, H. R - - $182-218,183-487$

Johns, R. K. C - 182-245

Johnson, J. C - 180-382

Johnson, H. M -

Johnson, R. W. , Jr -

Johnston, J. E -... 182-215

Jones, A. R - 183-526

Jones, L - -

Jones, O. S -... 180-45

Jones, V. L -

Judge, D. L - 183-425

Jurig, Karl -... 181-253

Jurain, Georges -....- 183-515

Kalashnikov, A. G--.-- 181-373, 183-429

Kalinin, V. A _...... 181-321

Kalinina, T. B -

Kamamoto, H -

Kametani, Takuya -.......- 182-491 
Abstract

Kamitsuki, Akira - - - 180-59, 180-60 Kanaya, Hiroshi -...--- 180-348, 180-349 $180-350,180-353$

Kane, M. F --.- 181-274, 183-363, 183-486 Kaneko, Jun -... 183-578 Kaneko, Tetsuichi - Kántás, Karl -... 182-286 Kapitanov, Yu. T _......... 180-18 Kaplunov, A. I -...- 183-264 Kappelmeyer, Oskar -.--.---- 180-237 Karapetyan, K. I -..-_ 183-590 Karasik, A. M Karatayev, G. I - Karaveyev, F. M Kärki, P Karmaleyeva, R. M -...... 183-145 Kárník, VÍt -............ 182-92, 182-109 Karras, M -...- 181-409, 183-15, 183-165 Kartashov, N. P Kartha, T. D. K - 183-505 Kasahara, Keilch1 -.........-183-210 Kasatkin, D. P - 181-230 Kashkarov, L. L - 180-328, 182-450, 183-508 Kałpar, Milan _............ 182-418 Kataoka, Akeo -............. 181-483 Kataoka, Hisashi -........... 182-182 Kato, Yoshio -........-181-364, 182-399 Katok, A. P Kats, A. Z Katsura, Takashi Kaufman, A. A -.....-183-261 Kaula, W. M - 180-182 Kautzleben, H------- 182-379, 183-419 Kawabata, Y Kawai, Naoto -._-_-182-265, 182-409 Kawashima, Takeshi -.......- 182-490 Kazakov, G. A _........ 183-33 Kazinskiy, V. A Kazmi, S. A. A $\ldots \ldots \ldots$ 182-388 Keen, M. J Keevil, N. B - - 180-125 Keller, G. V - - 180-122, 180-123, 180-150 $181-212,183-268,183-307$ Kelly, S. F - - 180-105, 180-126, 180-306 Kennedy, G. C - $182-342$ Kent, B. H - 181-260 Kerr, A. D Kertz, Walter-181-351 Keunecke, O -...-180-319 Keylis-Borok, V. I -......- 182-120 Khain, V. Ye Khaldeyev, O. D Khalfin, L. A - 183-185 Khan, M. A - . 181-385 Khanayev, Ye. I _. 182-11 Kharitonova, V. Ya Khaskind, M. D - 180-239 Khaykovich, I. M Khayritdinov, R. K Khitarov, N. I -..... 181-229 Khokhlov, A, K Kholin, A. I - 180-336 Khormushko, S. P _....... 181-414 Khovanova, P. I Khramov, A. N Khramoy, A. I -
Abstract

Khutsaidze, A. L Kimball, D. S-181-358 Kimbell, C. L-_._. 183-565 Kimpara, Atsushi --_----180-263, 181-369 Kimura, Shigemasa-_-_-_-_- 183-566 Kindij, Eugene -... 180-366 King, A. J King, E. R-183-484 King-Hele, D. G - 183-314 Kinosaki, Yoshiro Kinoshita, W. T - $181-260$ Kinyapina, T, A …........ 180-41 Kirby, J. E Kirchheimer, Franz -........-183-510 Kirillova, I. V -------183-121, 183-122 Kirova, O. A - 181-59 Kishimoto, Yoshimichi -....... 182-364 Kisslinger, Car1 ----180-321, 183-536 Kistler, R. W -..... 183-12 Kistner, G G........... 181-57 Kivioja, Lassi-.............. 181-251 Kizawa, Takash1---_---182-134, 183-159 Klemic, Harry -.......... 183-16 Klíma, Karel-...... 180-98 Klushin, I. G-....... 183-357 Klussmann, J Knapp, D. G . 182-378 Knopoff, Leon ----180-87, 180-241, 181-112 $181-155,181-322,182-162,183-150$ Knoppe, K. G - 183-37 Knorre, K. G -...-180-4, 182-44 Knox, F. B - $182-449$ Kobayashi, Hajime -.... 180-130 $180-134,180-167$

Kobayashi, Hisanobu -.......-183-529 Kobayashi, Kazuo -_.......-180-278

$180-290,181-391$

Kobayashi, Naota------181-134, 181-138 Kobranova, V. N - . Kŏcí, Alois - Kodama, Masahiro -.........-- 183-427 Koefoed, O -.-.-.-_--181-435, 183-215 Kogan, P. M Kogan, R. M Kogan, S. D - - 181-128, 183-206 Kogan, S. Ya Koide, Minoru -... 181-329 Kojima, M - . 180-407 Kojima, Seishi - 180-348, 180-349, 180-353 Kolbenheyer, Tibor -......... 181-186 $182-376,183-217$

Kollar, F -... 183-409 Komai, Jiro -..-180-348, 180-349 $180-351,180-352,180-353$

Komarov, A. G - 183-428 Komlev, L. V $183-35,183-36,183-38$

Komovskiy, G. F - 181-20 Komura, Saburo - 182-119 Kondo, Minoru -..182-399 Kondorskaya, N. V-..---181-102, $182-93$ $182-100,182-115,182-126$

Kondrat'yev, O. K -... 182-507 König, H -....... 181-63, 182-6, 183-74 Kon'kov, A. A - 180-42 Kon'kov, A. T - 183-126 Końo, Michiya -.... 182-470 
Abstract Konstantinova, A. G - $180-91$
Kopecký, Miloslav Korostin, G. N N Kortsenshteyn, V. N Koryakin, Ye, D - 181-306 Korzhev, A. A Koshlyak, V. A -... 182-336 Kosminskaya, I. P _... 180-166, 180-244 $182-344,182-356$

Kostenko, N. P Köster, Rolf -........ 183-342, 183-347 Kotlyarevskiy, B. V _....... 181-289 Kotyakhov, F. I Koulomzine, T -.... 180-303 Kovach, R. L _.... 180-64 Kovalev, O. I -.. 183-567 Kozhina, T. K Kozlenko, S. P - _._- 181-218, 181-220 Kozlov, A. B -.... 180-67 Kozlov, E. A Kozlov, P. T -.. 181-217 Kozyrin, A. K -..-.-. 183-236, 183-239 Kramer, M. V -..... 180-70 Kraskovskiy, S. A Kratts, K. O _........ 182-48 Kraus, E. C --- 180-198, 182-260, 183-321 Krayev, A. P _. Krestnikov, V. N Krishnan, M. S Krivoy, H. L Krog, Harald -.-1 183-28 Kropotova, O. I - Krs, Miroslav -_. Kruglyakov, V. V-.......... 182-408 Kruglyakova, G. I Krumbein, W. C Kruszewski, Zdisław -.....-183-231 Krylov, A. Ya -....- 181-50, 182-12 $183-40,183-46$ Krylova, M. D Kuchina, G. N Kudymov, B. Ya Kuhn, P. J -... 181-472 Kukharenko, N. K Kukhtikova, T. I -... 180-39 Kulikov, S. A -.... 181-462 Kulinkovich, A. E Kulp, J. L -...-181-1, 181-28,181-29 $182-2,183-2,183-506$ Kume, Shoichi _... Kun, V. V - - 182-156, 183-184 Kunetz, Geza - . Kuno, Hisashi -- 180-290, 181-312, 181-391 Kunori, Shoichi -.... 182-194, 183-230 Kunz, B. F. J Kupsch, W. O-... 181-33 Kurbanov, M -..... 181-100 Kurihara, Shigetoshi -_._-_-_-181-449 Kurimoto, Hiroshi -.... 181-109 Kuroda, Hidetaka - Kuroda, P. K -..... 183-7, 183-8, 183-70 Kushiro, Ikuo -. 183-451 Kuz'mina, L. A -.... 181-18 Kuznetsov, V. P Kuznetsova, I. M - 183-580 Kvasha, L. G _... 180-25
Abstract

Labeyrie, Jacques -

LaCoste, Lucien -..... 180-224, 181-268

Lacroute, Pierre -

Ladd, H. S - .

Lagrula, Jean -...-181-234

Lake, S -...

Lal, Devendra -...-.- 181-329, 181-420

Lambert, R. St. J -_. $181-1$

Landauer, J. K -180-208, 180-210, 180-211

Landisman, Mark-181-124, 182-159, 182-160

Lang, J. W -

Langhoff, $\mathrm{J}$-.... 182-70

Lapina, M. I -

Larionov, O. V -

Latter, A. L - . 181-415, 181-416

Latus, T, J - . -

Laubenbakh, A. I -

Laughton, A. S - 181-166

Lavergne, Michel --_---182-163, 183-539

Layat, C -

Lazarev, G. Ye -

Lazarev, K. F -...... 183-11

Lebedev, V. I -

Ledent, Dolly -.... 180-9, 182 -45, 182-46

Ledoux, Y -

Lee, M. R - 180-379

Lehmann, Inge --180-57, 182-363

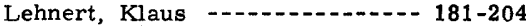

Leighton, M. M - 183-17

Le Moüel, Jean -

Lensen, G. J -... 180-201

Leonov, N. N -

Leont'ev, V. M -.. 180-139

Leparskaya, N. D - . . . 183-308

Lerici, C. M -.... 181-214

Levanto, A. E - $183-466$

Levin, B. Yu -

Levshin, A. L -

Levskiy, L. K - 181-331, 182-65

Licht, A. L -

Lill, G. G - $181-311$

Lindholm, T. M -

Lindsey, J. P -... 180-361

Lin'kova, T. I - $183-464$

Lipson, J. I ................ 183-1

Lisitsyn, A. P - ...... 180-400, 181-491

Lisowski, A -.......... 180-89

Lockhart, L. B. , Jr - 180-331

Loh, R. P - . 180-319

Lomnitz, Cinna -.............. 183-378

Long, A. M -

Long, L. E - $181-1$

Longman, I. M -- 180-71, 183-175, 183-292

Lorch, Siegfried -... 182-463

Lossovskiy, Ye. K -..... 181-126

Loughman, C. J -

Lovering, J. F Lovisyus, A. V-- 180-246, 181-333, 183-46

Lovtsyus, G. P $183-46$

Low, J. H - 180-299

Lowdon, J. A -... 182-35

Lowrie, J -... 181-368

Lozhnikova, O. N - 181-20

Lozinskaya, A. M--_-_-- 180-225, 183-474

Lucke, O -.....-- 181-342, 182-343 
Abstract

Lukasc, T -

Lundberg, Hans --_-_- 180-124, 182-186

Lundelius, E. L _...... 183-43

Luskin, Bernard -. 183-583

Lyakhov, B. M -

Lyamzina, G. A -

Lyatkovskaya, N. M -

Lyon, C -

Lyubimova, Ye. A - - - 181-297, 181-318

Lyustikh, Ye. N - . - 181-244, 182-259

$183-176,183-394$

Ma, En-tsze - - - 183-550, 183-551

$\mathrm{Ma}, \mathrm{T}$. Y. H -

Mabey, D. R -

McAulay, I. R-_..... 181-16, 182-445

McBirney, A. R _..... 181-492

McCall, G. J. H

McCallum, K. J _....... 182-25

MacCarthy, G. R -

McCollum, E. V-1.. 180-171

McCrossan, R. G -.... 180-143, 183-270

McCulloh, T. H - . . 183-374

Macdonald, G. A -

MacDonald, G. J. F --_--181-119, 181-155

$182-257,182-287,182-358$

McDonald, J. E _.

Macdonald, N -

Mac Dowall, J -........... 180-323

MacFarlane, R. M -

McKellar, I. C ..... 183-44

McKenna, S. M. P - 183-445

McKeown, F. A - -

Mackey, C. J - 182-21

McLaughlin, Rowland -..-_- 180-383

Macpherson, J. D -........ 183-569

Maeda, Hiroshi -...... 180-263, 182-397

Maere, X. de -_. 183-402

Magnitskiy, V. A - ...-- 181-321, 183-176

Magnusson, N. H -

Magrath, C. B - $183-570$

Maino, Armando -

Mair, J. A - 181-36

Major, Maurice -

Makarova, Z. V -

Maksimov, S. P _..... 181-21

Malakhov, S. G _.... 181-419

Malaroda, Roberto _........ 182-91

Malinovskaya, L. N -

Malov, N. N - 183-596

Malovichko, A. K -

Malurkar, S. L -.... $182-443$

Mandel'baun, M. M -

Mangadze, G. D -

Mann, R. L - 181-162

Manning, G. K _.. 182-67

Maple, Elwood -- 183-446

Marcelli, Liliana _....... 181-106

Mardock, E. S - _... $182-457$

Mariani, F - - 182-393, 182-394, 183-448

Maringer, R. E ...... 182-67

Marini, G -

Markhinin, Ye. K -..-- 181-390, 182-543

Markova, N. G _... 181-17

Markowitz, William -_...-181-149

Marmo, Vladi _... 182-7
Abstract

Martin, Hans -...-. 183-207, 183-208

Martin, L. H -

Martin, Maurice -182-200, 183-303, 183-304

Martin, W. R. B - 183-511

Martinez, J. D - 183-461

Martishchenko, L. G -

Marussi, Antonio-_-_ 183-383

Mason, Brian -...- 181-53, 183-52

Mason, R. G - 182-349

Mason, R. J -

Masse, G. W - $182-265$

Masuda, Hideo -

Mathews, W. H -

Mathieu, J. L ---181-278, 181-395, 183-252

Matsubara, Hideki _._....... 182-469

Matsuda, Takeo -...... 180-218, 183-384

Matsumoto, Hatao -...-_-_ 182-535

Matsumoto, T -

Matsuo, S -...-180-411

Matsuoka, Bun-ichi -.......-182-182

Matsushima, Shogo --_---180-396, 182-171

Matsushita, S - 181-363, 182-396, 183-437

Mattei, J. B -... 180-172

Matthews, S. W -...... 181-86

Matuzawa, Takeo-.... 180-387

Matveyev, B. K - $181-180$

Mavritskiy, B. F - - - 180-21, 181-299

Mayne, K. I -.... 183-74

Maynes, A. D - 181-36

Meade, B. K - . 180-192

Medvedev, S. V -....... 180-69, 182-94

$182-111,183-114$

Meidav, Tsvi -....... 183-245, 183-553

Meier, M. F -

Meigh, A. C -

Meisser, Otto -...-183-301

Melchior, P. J - 180-74

Melik-Shakhnazarov, A. M -.--- 183-266

Mellor, Malcolm -.... 180-215

Mel'nikov, A. G-183-266

Melton, B. S - 183-164

Menard, H. W - $182-522$

Mendonça Dias, A. A. de -.... 180-402

Menzel, Heinz -.. 180-84

Mériel, Yves -.... 182-541

Merlini, E -... $181-457$

Mero, J. L -

Merrill, W. M _.... 183-350

Meshcheryakov, Yu. A -...... 182-271

Messmer, J. H - 183-389

Metallova, V. V - 180-270

Metzger, A. A. T - 182-179

Metzger, Joseph - $182-131$

Mey, Shi-yun --

Miguel y Gonzalas Miranda,

Luis de -

Mikhalevskaya, A. D - -... 180-5, 180-6

Mikhaylov, N. N -

Mikhaylovskiy, V. N-183-472

Mikhota, G. G - 180-224

Mikumo, Takeshi-_-_...- 180-54, 180-100

Milea, Nicolae -...-180-256

Miles, J. W -... $182-154$

Millard, F. S - - $181-224,182-498$

Miller, D. E - . 180-243

Miller, D. J - 
Abstract

Miller, G-_... 183-594

Miller, J, A -......... 183-20

Millican, M. L Mills, A. A -...... 181-1 Milton, B. E - - -180-395, 182-229, 183-251 Minakami, Takeshi _........ 182-538 $182-539,182-540$

Mindlin, L. E - _... Minton, P. D Mishin, V. M -.... 181-83 Mitra, Manindra --.--- 183-182, 183-186 Miura, T -..... 182-143 Miyachi, M Miyachi, S -........... 180-407 Miyazaki, Tsutomu -_._-_._-_ 182-539 Mizutani, Y _........ 180-411 Mizyuk, L. Ya _...... 182-196 Model', Yu. M -... 183-290 Modriniak, N -....... 180-170 Mogi, Kiyoo - Moiseyenko, F. S Mokhova, Ye. N Molina, F -...- 182-393, 182-394, 183-448 Molochnikov, Z. I -._-_._...-- 181-206 Molochnov, G. V-...... 180-112, 181-184 Molotov, L. V -..... 183-567 Monakhov, F. I _....... $182-440$ Moody, R. C _........ 183-545 Mooney, H. M Moore, D. G ..... $183-584$ Moore, G. W Moore, J. M. , Jr Moore, Patrick -........... 182-77 Moore, T. F - $180-173$ Morales, L. G - 183-281 Morelli, Carlo --..-- 180-160, 181-267 Mori, Kyoshi -... 180-384 Morimoto, Ryohei -_-_-_-_-183-595 Morley, L. W Morozov, G. S - 180-136 Morozova, I. M -...... 183-13 Mosetti, Ferruccio - Mouton, Jean -. Moxham, R. M Moyd, Louis - 180-302 Mozzhenko, A. N - .... 181-446, 181-461 Muehlberger, W. R Mueller, Stephan Mumme, I. A -....... 182-309, 182-325 $182-474,182-466$ Munk, W. H Münnich, K. O -... 182-17 Muraoka, H Murata, K. J-_... Murin, A. N - 180-24 Murina, G. A - - - 181-13, 181-46, 183-33 Murozumi, Masayoshi _....... 180-133 Murphy, L. M Murphy, Thomas - Murtazina, T. M - 180-252 Musgrave, A. W Mustafayev, K. A - - 183-285 Myachkin, V. I-_. 183-194 Myers, W. H -... 180-381
Abstract

Nabeoka, S -.

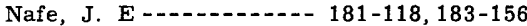

Nagata, Takesi -...... 180-290, 180-291 $181-391,182-416$

Nagumo, Shozaburo -

$182-173,183-187$

Nairn, A. E. M ....... 180-281, 180-289

$181-383,181-386$

Nakai, Junji--_-_-_-_-_ 180-356, 180-359

Nakamura, Hisayoshi - . - . . - 180-413

$181-501,182-544$

Namba, Munetosi -

Nanda, J. N----180-242, 180-367, 181-403

Narans, H. D. , Jr -.... 183-395

National Academy of Sciences --- 181-308

Nauta, H -

Naydenov, B. M -....... 183-410

Nedostup, G. A - 180-336

Neelov, A, N - $182-50$

Nekhoroshev, A. S -.... 182-542

$\mathrm{Nel}, \mathrm{L}, \mathrm{T}$

Nelepo, B. A

Nelson, J. H - $181-341,182-378$

Németh, Ferenc -........... 180-195

Nepesov, R. D - 181-100

Neprochnov, Yu. P _... 180-376

$180-392,182-504$

Nersesov, I. L -------182-96, 183-125

Ness, N. F - 182-358

Nesteroff, W. D $\ldots 182-47$

Nesterov, I. I -

Nettleton, L. L - - - - 180-224, 183-376

Neugebauer, Marcia _........ 183-88

Neumann, Frank -182-110, 182-144, 182-351

Neumann, W -....... 182-425

Nevolin, N. V -

Newman, B. W -

Newstead, Gordon - - 182-138

Niblett, E. R -...... 183-92

Nicholson, S. B -

Nief, G -...- 181-327

Nielsen, Hardy -.... 180-148

Nier, A. O -.......... 180-23, 182-63

$182-72,183-60$

Niino, Hiroshi -...... 183-513

Nikiforova, N. N -

Nikitenko, K. I - - 183-96

Nikitin, M. K - 180-332

Nikitin, P. N -

Nikitin, V. N -

Nikitina, V, N - - - 183-224, 183-256

Nikolov, N. S -

Ninzhbadgar, S -......... 182-101

Niskanen, E - -

Norinelli, Armando--.-- 182-285, 182-305

Norris, R. M -... 181-489

Nosske, Gerhard-181-200, $182-195,183-283$

Novozhilova, M. Ye-----180-117, 180-120

Nudel'man, A. V - 183-290

Nurmia, M------181-409, 182-361, 183-15

$183-165,183-169,183-397$

Nuttli, O. W - 183-100 Nydal, R Nye, J. F - 180-206, 180-207 
Abstract

Oakeshott, G. B Obayashi, T -....... 180-263, 181-372

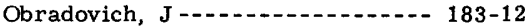
O'Brien, P.N. S -181-174,182-485, 183-554 Obukhov, V. A - - - 181-145, 181-458 Öcal, Nevzat Ochaba, Štefan -....... 181-399 Odani, Yoshitake -.-.-.- 180-132, 180-133 $180-168,180-169$ Officer, C. B. , Jr Oganisyan, Sh. S -181-290, 181-291, 182-324 Ogawa, Kenzo - 180-354 Oguchi, Yuko Oguri, Mikio -....-181-483 Ohashi, Shuji Ohm, J. M Oil in Canada ......... 180-152, 180-338 $180-378,182-222,183-280$ Oilweek -.....- 180-343, 181-453, 183-571 Okano, Kennosuke -.........- 182-441 O'Keefe, J. A -..--- 180-183, 180-184 Olberg, Manfred -..........-- 181-252 Oldham, C. H. G Olhovich, V. A -----182-475, 183-543 Oliver, H. W -..... 183-373 Oliver, J. E ----181-118, 181-130, 182-141 $182-174,183-154,183-203$ Olson, E. A Olsson, Ingrid -..... 182-28 Olszak, G -.... Omar, H. M Omori, Keiichi -....... 182-60 Ono, Nobuo - . 181-486 Ono, Yoshihiko --180-167, 182-183, 182-205 Onodera, Koji -..... 180-132 Onodera, Seibe Onwumechilli, C. A _ - . . .

$182-386,183-424$

Opdyke, N. D -

Opik, E. J -..... 183-90 O'Reilly, John - 180-34 Organova, N. M - 183-130 Orlin, H - 180-180 Orlova, L. P _... 181-5 Orr, P. C Ortynski, H. I Ose, Masami Oshima, H -.... 180-265 Osokina, D. N Ostapenko, V. F -.... 180-328, 182-450 Ostenso, N. A Östlund, H. G -.... 182-30,182-372 Ostrovskiy, V. D-.... 180-163 O'Sullivan, J. B -............ 183-18 Oszlaczky, Szilárd Otaki, Tadao Oudelette, A Ovchinnikov, L. N-........ 183-39 Overholt, K. J -..... 183-216 Owa, Eijiro-..... 182-468

Paarma, H -... 183-466 Pachadzhanova, G. N Pak, V. A - ... 182-505 Pakiser, L. C ---181-240, 181-274, 183-586 Pallister, A. E -..... 181-453
Abstract

Pan, Chia-Lin ..... 182-530

Panasenko, G. D Paneth, F. A - 183-74 Pankina, R. G _.... 181-21 Panner, Norbert - . Parasnis, D. S - -180-113, 181-485, 183-223 Pariyskaya, G. N - 183-184 Pariyskiy, N. N - $180-70$ Parker, E. N $181-355,183-432,183-440$ Parkhomenko, E. I -........ 181-169 $181-208,182-214$

Parkin, D. W Parkinson, W. D --.... 180-255, 181-348 Parsons, G. E - 180-304 Parwel, A -...-183-407 Pasechnik, I. P --180-44, 181-128, 181-144 Patchett, J. E-... 181-36 Paterson, N. R ....... 181-201, 183-223 Paton, J. R Patrick, H. G Patten, E. P. , Jr Pavlov, B. S -.... 180-240 Pearn, W. C Pecherskiy, D. M - 181-389 Pěčová, Jana -... 182-391 Pelletier, Henri Pemberton, Roger Penttilä, Esko ----------183-115, 183-397 Per'kov, N. A Perquis, M. T - 180-20 Perret, W'. R -

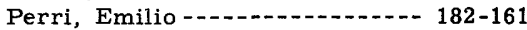
Pertsev, B. P Peter, G -. $183-469$ Peters, B Peters, J. W Peterson, P Petkevich, G. I - 183-552 Petr, Vaćlav Petrov, Ye. N Petrushevskiy, B. A - . 180-46, 181-103 $182-94,183-143$ Petryayev, Ye. P _.... 183-11 Petrzhak, K. A -.- 181-62 Péwé, T. L Philip, J, R Phillips, C. A., Jr-an 181-377 Picciotto, E. E - - 180-249, 183-402 Pícha, Jan -..... 182-148 Pickell, J. J Pickett, G. R Pilant, W. L Pinson, W. H. Jr -.... 181-24, 181-26 $181-38,181-73,182-36$ Plakhov, Yu ........ 180-18, 180-190 Plesset, M. S -..-_-- 181-415, 181-416 Plokhikh, N. A -... 183-233 Plouff, Donald -............. 181-212 Poder, Knud -..... 183-317 Podgornaya, N. S -........... 182-51 Pod"yapol'skiy, G. S --- 181-127, 181-156 $181-157,181-437$

Pohrte, T Pokshivnitskiy, Ye -........-. 183-53 
Abstract

Pola, Ivan --..-- 182-292, 182-293 $182-294,182-319$

Poldevaart, Arie Poldini, E Polevaya, N. I -_-_-_- 181-46, 182-51 $182-52,183-33$

Polikarpochkin, V. V - Polkanov, A. A -... 183-31 Polonskiy, A. M Polshkov, M. K Polyakov, A. B - 183-366 Polyakov, Ye. A Pomerants, L. I - - . Pomerantz, M. A Pomeroy, P. W -181-142, 181-174, 183-203 Pomîrleanu, V Pommier, Gilbert -.....-...-183-573 Poole, J. L Popov, G. I Popov, Ye. I--.- 181-270, 182-306 Popov, Yu. N Porkka, M. T-2-182-361, 183-399 Porstendorfer, Gottfried --.---- 181-78 Pospelova, G. A -180-288, 181-390, 182-413 Postolenko, G. A - 182-100 Potnis, V. R Powell, D. W - 183-453 Prentiss, David --_.--183-189 Press, Frank -. 180-147 181-141, 181-154, 181-274 $181-305,181-315,182-135$ $182-149,183-88,183-191$

Preston, R. G - 182-175 Priroda -...... 183-87

Prokof'yev, F. N - 180-336 Prout, James -... 180-383

Puchkov, S. V -

Pudovkin, I. M -..... 182-429, 182-430

Pushkov, N. V -

Pustil'nikov, M. R -... 180-161

Pyatchin, N. F - 182-506

Quadir, M. A - $181-479$ Quarterly Journal

Seismology -. 182-88

Quennell, A. M - 183-3

Rabinovich, A. V - M 180-252

Radice, M. M - $183-56$

Radu, C - 181-107

Radzhabov, M. M - 180-369

$182-477,182-478$

Raimondi, Carlo -..-_ 180-160, 182-91

Raitt, R. W -

Rakitov, A. I -- 180-164

Rama ......... 181-420, 183-408

Ramazanzade, M. G - .

Ramirez, J. E -... 180-404

Rankin, P. A -

Rao, B. R - 181-164

Rao, B. S. R - $182-405$

Rao, K. S. R -

Rao, M. B. R - 180-129, 180-312

Rao, M. N. S -

Rao, S, H -

Rasool, Ishtiaq -
Abstract

Raspopov, O. M - - 180-216, 183-421

Rastvorova, V. A -

Ratcliffe, E. H - 181-300

Ratcliffe, J. H - 180-305

Rausch, D. O - 180-212

Ravich, M. G - 183-46

Reid, J. H - $183-445$

Reilly, W. I -

Remington, E. W - 183-491

Renner, János -...-- 181-282, 182-322

Repsold, Hans -... 180-30

Research Group for Explosion

Seismology [Japan] -....... 180-99

Research Group for Spontaneous

Polarization Method [Japan] -..- 183-250

Reusche, H. -G -

Revelle, R. R - 181 -487

Reynolds, J. H _....... 181-334, 183-66 $183-67,183-68$

Reyzner, G. I -

Rice, D. A - 180-186

Richard, Henri -

Richards, A. F -

Richards, T. C -.... 181-443, $181-459$

Richardson, H -

Richmond, Jean - . 183-155

Richter, C. F - - 180-81, 183-101, 183-102

Richter, D. H -

Richter, Gertraud - 181-139

Rigsby, G. P $P$ - 182-275

Rilhimaa, J -.... 183-211

Rik, K. G - $181-47$

Rikitake, Tsuneji -..-180-266

Riley, G. H - $182-55$

Rimmer, W. G - 183-561

Rinehart, J. S - $182-510$

Ringwood, A. E -- 181-56, 183-54, 183-400

Rische, Hans -... 181-436

Rittmann, Alfred -...- 182-528

Riznichenko, Yu. V-_--180-372, 181-171

$182-344,183-193$

Robbins, E. J -

Roberts, Archie - . . 183-583

Robertshaw, Jack----180-386, 180-390

Robertson, E. I -

Robin, G. de $Q$ -

Robson, G. R -

Rocard, Yves -.....-181-175, 181-176 $182-436,182-444$

Roche, Alexandre -...- $182-412$

Rochester, M. G - 183-430

Rodionov, P. F- 180-116

Rodionov, S. P - 183-34

Roeschmann, Fritz _.... 182-197

Roethlisberger, Hans -...-.-- 182-502

Rokityanskiy, I. I -

Roller, J. C -

Romney; Carl -.........-180-96

Roques, Maurice ---183-23, 183-24, 183-26

Roquet, Juliette - 182-401

Rosenbaum, J. H -........... 181-152

Rosenfeld, J. L -

Rostomyan, P. M - 183-390

Roth, E -

Roubault, Marcel - 183-509

Roux, A. T - 
Abstract

Rowell, J. A - _....... 182-359

Roy, Amalendu --180-220, 183-278, 183-354

Roy, Rustum--.-.-181-313

Rubin, Meyer ---------182-29, 182-34

Rubinshteyn, M. M -

$182-13,182-14,183-120$

Rudel, Aimé -..... 180-20

Rudich, Ye. M -...... 183-132

Rudman, A. J - ..... 183-568

Runcorn, S. K -

Russell, R. D -

$183-375,183-409$

Russell, W. L -

Rustanovich, D. N - . .

Rutten, M. G - 183-463

Rưžička, Jaroslav ............. 182-294

Ryabinkin, L. A - 182-241

Ryabukhin, G. E-182-337

Rybakova, Ye. V -180-110, 181-179, 181-195

Rybin, A. I -

Ryhage, R -

Rykunov, L. N - 181-132

Sachs, D. C - 182-175

Sachs, Maureen --.-183-78

Safaryan, A. N-18

Safontsev, A. A -...-183-258

Safronov, N. I -

Saha, B. P _... 183-504

Saito, Takao - ..... 182-399

Saito, Tomosaburo -........ 180-275

Sakovtsev, G. P--.---183-234, 183-235

Sakuma, Shuzo -

Sakurai, Takehisa -...-182-167

Salikhov, A. G - 183-382

Salvioni, Guido-------182-270, 182-314

Sanders, N. K - 183-104

Sandström, A. E -

Sanselme, H -...-180-149

Särchinger, H -

Sarcia, J -

Sardarov, S. S ---180-14, 181-14

Sargent, J. D -

Sastry, Ch. V -

Sato, Hisashi -

Sato, Motoaki -......-180-114

Sato, Ryosuke -..-182-166

Sato, Yasuo -..-18-180-63, 181-124

$182-159,182-160,183-190$

Savarenskiy, Ye. F------180-48, 181-99

$181-117,182-103$

$182-114,182-115,182-481$

Savinskiy, K. A -

Savit, C. H - 180-364

Savonenkov, V. G ---180-6, 183-34, 183-35

Savul, M -..... 181-302

Sawata, Hideho-_-_-_-_ 183-591

Sawatzky, H. B- 182-224

Saxov, S. E - 183-300

Sayn-Wittgenstein, C -

Sazhina, N. B-C 181-263

Scala, C - .................. 180-140

Schad, Albert -

Schaeffer, O. A -..-_-_-182-69, 182-73

$183-61,183-65$

Schaller, H. E -
Abstract

Scheidegger, A. E - - 181-247, 182-121

Schlanger, S. O -

Schmidlin, $P$-----181-65, 183-64, 183-74

Schmidt, R. G -

Schmucker, Ulrich-180-286, 181-80, 182-82

Schneider, Götz - .

Schofield, J. C -...-_- 183-45, 183-323

Scholte, J. G. J - 181-371

Schössler, Klaus - $\ldots \ldots \ldots$ 183-386

Schürmann, H. M. E --.- $181-44,182-46$

Schwab, R. F _... 181-396

Schwaetzer, T -

Schwarzlose, Jobst . . . . . . . . 183-386

Schwind, J. J - .

Scott, H. S -

Seedsman, K. R -

Segesman, F -

Seigel, H. O - . . 180-119, 180-155

Seino, Masaaki -..._........ 180-412

Seitz, Konrad -........ $182-463$

Sekiya, H -

Sell Cantalapiedra, J. I _........ 183-247

Selzer, Édouard -........ 182-401

Semenenko, N. P . .

Semenov, A. A _....... 180-40

Semenov, A. S - - - 180-120, 182-181

Semenyushkin, I. N -

Senftle, F. E -

Sengbush, R. L - - 180-365, 182-494

Sen'ko-Bulatnyy, I. N -

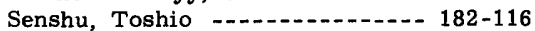

Serata, Shosei - -

Serdyukova, A. S .......... 180-18

Serrano, E. S -

Service Hydrographique de la

Marine and Companie Générale

de Géophysique -....... 180-72

Seya, Kiyoshi _...... 180-352, 182-300

Shabanov, B. A -

Shakhsuvarov, D. N -

$181-179,181-197$

Shale Shaker -

Shamina, O. G -.... 180-92, 181-170

Shand, J. A -

Shan-pan, Li -

Shapiro, I. R-_... 181-340

Shapiro, Ralph -

Shapiro, R. P -

Shats, M. M -... 181-162

Shayins'kyy, O. M -

Shebalin, N. V -

Shekht, N, I -

Sherwood, A. M -

Shibato, Kihei -- 180-118, 180-168, 183-244

Shima, Etsuzo - - .

Shimizu, Yoshio -...... 180-291, 182-416

Shirokova, Ye. I -...... 181-115

Shkabarnya, N. G - 181-180

Shmarova, V. I -.... 183-219

Shmidt, O. Yu -

Shmonin, L. I - -

Shneyerson, B. L - $181-400$

Shneyerson, M. B -

Shoemaker, E. M - - 183-76, 183-202

Shor, G. G. Jr - 183-396

Shukolyukov, Yu. A --180-10, 181-15, 181-46 
Abstract Shumskiy, P. A - 180-234 Shumway, George -...- 181-165, 182-482 Shurbet, D. H Shurkin, K. A -..... 182-49 Sidorov, V. A Signer, P -.......... 183-60, 183-72 Siivola, A -...-183-170 Silin, Yu. I -.......... 181-50, 182-12 $183-40,183-46$

Silva, Giovanni -... 182-305 Silver, L. T Š́imon, Zdenĕk -............... 182-315 Simonen, Ahti .... 183-30 Simpich, Frederick, Jr - _...- 181-495 Simpson, J. A Simpson, T. A - 182-85 Singer, S. F - - - 182-373, 182-390, 183-90 Sinha, S. C Sinitsin, A. P Sinno, K Sirin, A. N Sivaramakrishnan, M. V -..... 182-54 Sivaraman, K. R Skipp, B. O - Skorupa, Jan - - 183-284 Skosyreva, L. N _....... 181-429 Skougstadt, M. W Skugarevskaya, O. A --_-_--- 181-195 Slichter, L. B - - - - - 182-146, 183-279 Smellie, D. W-- 180-295, 180-316, 182-220 Smirnov, A. M Smith, Carl _....... 181-411 Smith, E. J Smith, F. G - 180-125 Smith, W. E. T -.... 180-32 Smith, W. I _. Smyslov, A. A Snow, Leland Sobotovich, E. V ..... 180-246, 181-333 Sokhranov, N. N Solonenko, V. P - . - - 183-113, 183-128 Solov'yev, S. L - 180-50, 181-105, 182-112 Sonett, C. P Sorokhtin, O. G Sorskiy, A. A South African Mining

Engineering Journal -.....- 182-423 Sparrer, H Spencer, A. J. M Spencer, T. W Sponheuer, Wilhelm _....... 183-208 Sprague, G -..... 181-358 Sprigg, R. C Sprintsson, V. D - 181-46 Srinivasamurthy, B - . Stacey, F. D-- 182-403, 182-407, 183-454 Stackler, W. F Stackler, W. G-183-367 Stakhovskaya, Z. I - Stam, J. C -..... 183-467 Starik, F. Ye - 183-11 Starik, I. Ye - - - 180-246, 181-4, 181-62 $181-333,183-11,183-14,183-46$ Statham, E. H - 183-461 Stauder, William -..... 181-92, 183-107 $183-138,183-139,183-140$
Abstract Stauffer, H Stearn, J. L - ... Steenland, N. C - 182-424 Steensma, J. J. S -....... 182-46 Stefănescu, S. S-_._.-. 181-189 Şteflea, Ligia -.... 180-256 Steflea, Vladimir Stegena, Lájos -.......- 182-267, 182-334 Stehli, F. G - $181-241$ Steinbrugge, K. V - 183-335 Stelzner, Johannes -.....-183-152 Stenko, V. A - 183-57 Stepanov, V. P -...-. 182-428, 183-382 Stephenson, P. J Stern, T. W - 180-8, 183-16 Stevens, C. M - 183-63 Stewart, S. W Stick, J. C. Jr -... 182-459 Stieff, L. R -...... 180-8, 183-16 Stoenescu, Scarlat 180-229, 181-285

Stolarik, J. D Storey, L. R. O - 180-258 Stott, P. M - 182-407 Stovas, M. V -.... 180-52 Stoyko, Nicolas-_...-.-.-.-181-148 Strakhov, V. N Strauss, M. G Strenger, H Strick, E Stride, A. H -... 181-166 Strobach, Klaus Strong, H. M-Stroud, S. G - 180-333 Studenikova, Z. V -..... 180-4 Studt, F. E Stuiver, Minze - 182-23 Stumpff, Karl _... 183-298 Stupnikova, N. I - 183-32 Styro, B. I - - $182-452$ Subbarao, K-.............. 181-164 Suda, Yoshiro--. Sudovikov, N. G - Suess, H. E Sugiura, Masahisa _........... 183-441 Sugiyama, Kosuke -....... 180-133 Sugiyama, Mitsusuke--_..-..-180-131 Sugiyama, Tomonori _......... 180-351 Sukhodol'skiy, V. V-_._- 181-269 Sukhov, I. M - 183-112 Sulin, V. V ----180-337, 181-422, 181-426 Sultanov, D. D Sultanov, F. S - 180-37 Sultanova, Z. Z -.... 182-95 Sungurov, A. M - 180-162 Surazhskiy, D. Ya -..... 182-467 Suringa, R-182-46 Sutton, G. H - --180-202, 181-142, 182-141 Suwa, Akira -...... 180-410 Suyama, Junji --..---180-130, 180-131 $180-134,180-167$ Suyama, K--...- 183-555 Suzuki, Ziro Svatkov, N. M-183-351 Svejgaard, B. J Svetov, B. S - 181-182 
Abstract

Svoboda, Karel -....- 181-344

Svyatlovskiy, A. Ye -_...... 180-403

Swift, Gilbert -... 180-141

Szemerédy, P - - 182-169

Szilárd, József --_-_-_--181-282, 182-321

Sztompke, WacYaw -.......-. 181-235

Tabata, Tadashi--- - - - 181-486, 182-521

Tabulevich, V. N--.---180-322, 183-503

Tajima, Hirokazu-_.

Takagi, Shinichiro-----180-130, 180-132

$180-169,183-249$

Takahashi, Hiroshi -

Takahashi, Tan --180-350, 180-358

Takeuchi, Hitoshi -... 181-134

$181-315,181-338$

Tal'virskiy, D. B -

Talwani, Manik ------180-191, 180-217

Tal'yanskiy, I. I -

Tamers, M. A -

Tanaka, Akiyoshi -...-180-354

Tanaka, Seizo-_-_ 182-535

Tanaka, Sadakatu -

Tandon, A. N -

Tanner, A. B ---181-413, 183-520, 183-523

Tarasov, L. S -... 180-247

Tarkov, A. P - 180-230

Tauber, Henrik ----182-17, 182-19, 182-20

Taylor, G. A-c 182-537

Taylor, J. H-_- 183-299

Taylor, S. R--.--183-78, 183-411

Tazieff, Haroun -.... 181-496

Teisseyre, Roman-----180-53, 181-114

Telfair, David -... 181-411

Temkin, A. Ya - $182-462$

Terada, Makoto -_.-182-167

The Hien, Tran -

Thellier, Émile - - - - 180-287, 181-339

Thellier, Odette -

Thiel, Edward -...--181-275, 181-293

Thode, H. G - $181-335$

Thomas, Erich -

Thomas, P. D - 182-254

Thompson, G. A-... 183-327

Thompson, L. G. D - --181-268, 182-302

Thorarinsson, Sigurdur _..... 182-529

Thorpe, Arthur -183-81

Thralls, H. M- 183-546

Thulin, Ake - .

Thyer, R. F - 182-326

Thyssen-Bornemisza, Stephen --- 183-367

Tikhonov, A. N-...--180-110, 181-179

$181-191,181-197$

Tikhonov, V. I --_-_--181-102, 183-585

Tillotson, Ernest -.......... 181-93

Tilton, G. R -

Timayev, K. V - 183-358

Timerbayeva, K, M-_-1-182-532

Timergazin, K. P _...... 181-48

Timofeyev, A. N -

Tixier, M. P----182-201, 183-303, 183-304

Tkachenko, A. A -

Tobyáš, Vladimír-.......... 182-92

Tocher, Don -

$183-103,183-106,183-336$

Tokunaga, Shigemoto-- 182-470
Abstract

Tolstikhin, O. N -

Tolstoy, Ivan -

Tomaschek, Rudolf - - .

Tomoda, Yoshibumi -----181-257, 181-258

Tongiorgi, Ezio - -

Torkhovskaya, L. N -

Towle, Guy -

Tozer, D. C - 181-207

Trapp, Erich-.-180-36

Treskov, A. A -.... 183-128

Trevisan, Livio -..........-181-225

Tskhakaya, A. D -

Troitskiy, V. N -

Trumbull, James -

Trushkov, Yu. N -

Tsitovich, A. P - 180-336

Tsuboi, Chuji -...-181-108, 181-250

$182-299,183-353$

Tsutsumi, Tokudo _.... 182-447

Tsvetkov, O. S -

Tudge, A. P -

Tugarinov, A. I - - - $180-7,181-5,181-47$

$181-49,182-44,182-467$

$183-34,183-35,183-37$

Tulina, Yu. V -

Tupper, W. M-_. $181-40$

Turkel'taub', N. M -..... 181-21

Tuttle, O. F - 183-393

Tvaltvadze, G. K - $181-307$

Tyapkin, K. F---181-255, 183-220, 183-360

Uchibori, Sadao -

Udintsev, G. B---180-376, 183-585

Ujiie, Akira -

$180-356,180-359$

Ukai, Yasuo-_..._-_..... 182-471

Ulomov, V. I - -

Ulrych, T. J -... 183-409

Umantsev, D. F -182-210, 182-430, 182-431

Umperovich, N. V . .

United States Air Force -..-...- 181-76

United States Coast and

Geodetic Survey -

Uotila, U. A. K --182-246, 182-247, 183-312

Urey, H. C $182-78,183-79$

Urquhart, D. F-a 181-431

Usami, Tatsuo - -

Ushakov, S. A -

Uspenskiy, D. G-_ 182-298

Utsu, Tokuji - $182-357$

Utzmann, Réné - 180-104

Uyeda, H -

Válek, Rostislav - $182-290$

Valiev, A. A -

Valle, P. E--

Vander Stricht, A-........ 182-16

Vaněk, Jiř́ - $182-109,183-152$

Van'yan, L. L-_... 180-108

Van Weelden, Arie-........ 182-227

Vasil'yev, V. G --.----180-232, 181-216

Vassy, Arlette -

Vaughn, W. W - $182-454$

Vdovykin, G. P- 183-59 
Abstract

Veis, George - - 182-255

Vendel'shteyn, B. Yu - - -181-187, 183-218

Vening Meinesz, F. A---180-178, 180-196 $180-197,183-330$

Venkatasubramaniam, V. S-...- 182-54 Verhoogen, John--_-_-180-267, 182-328 Verma, R. K - 181-161 Verö, J - . Versey, H. R - 180-127 Vesanen, E. E _... 182-361 Veselov, K. Ye Veshev, A. V - $\ldots \ldots 182-181$ Vestine, E. H Vetterlein, Pascal -......... 181-448 Veyl, G-_...... 183-594 Veytsman, P. S Viktorov, B. N-180-391, 181-477 Vilczek, Else -..- 182-64 Vincenz, S. A - Vinogradov, A. P--180-7, 180-247, 180-251 $181-17,181-47,181-58,181-60$ $181-66,182-44,183-35,183-37$

Vinogradov, S. D -..-- 180-90, 181-484 Vladimirov, N. P...-.--181-82, 181-192 Vlodavets, V. I --_-_--181-493, 181-497 Vogler, Gerhart - _. 181-196

Volarovich, M. P-_._-181-168, 181-208 $183-195,183-274$

Volland, Hans ............ 181-77

Volodarskiy, R. F - 180-231

Vol'vovskiy, B. S-_-_- 183-576

Vol'vovskiy, I. S -

Vorob'yev, G. G----181-74, 183-85, 183-86

Voshage, H -

Voskoboynik, N. I -.......... 180-142

Voûte, Caesar - . . . 180-340

Voyutskiy, V. S - - -

Vvedenskaya, A. V -----181-320, 183-142

Vvedenskaya, N. A _._._.... 182-98

Wada, Tatsuhiko-_- $-182-367,182-368$ Wadati, Kiyoo--.----182-105, 183-151 Wagner, W. R-_.-181 Wahl, W. G----180-296, 180-301, 183-468 Wait, J. R---182-177, 183-221

Wakai, Noboru -..... 183-450

Walker, Terry -.... 181-202

Wallerstein, George --.-180-209

Walter, E. J - ... 183-161

Wänke, H ----181-61, 182-6, 182-64, 183-74

Ward, F. W. Jr

Ward, S. H - --180-115, 180-121, 180-154

Wargo, J. G -... 181-381

Waring, C. L - 180-17

Warman, H. R - 181-209

Warrick, R. E -

Warwick, C. S - -

Washington Division

Mines and Geology - 183-531

Wasserburg, G. J -- - $-180-12,183-69$

W/atanabe, Hikaru -....- 182-142

Watanabe, Tomiya-_-_-181-364

Watt, P. A-18

W/eber, Max-_..- 183-544

Webster, R. K - $181-1$

Weihaupt, J. G- $182-230$
Abstract

Weiss, Oscar - 180-307

Wensink, H -..... $183-463$

Wescott, E. M ---182-81, 183-93, 183-94

Westgate, Robert -

Westrick, E. W - - - $180-297,180-304$

Wetherill, G. W --- $180-12,181-35,182-37$

Wheatley, G. Y

White, J. E -

Whitehead, J. D -

Whitten, C. A -

$183-114,183-337$

Whitten, E. H. T - 183-80

Whitten, G. F - $182-433$

Whitworth, V. L-_... 181-261

Widess, M. B -

Wijnen, J. C. van -----182-311, 183-377

Wilcox, J. B -

Wiles, D. R-183-524

Wilkening, M. H-_... 183-514

Williams, Howel-183-589

Williams, L. W -

Willis, D. E -

Willis, E. H -

Willman, H. B - 181-30

Willmore, P. L . . 182-140

Wilmarth, V. R -

Wilson, A. T-2 $181-324$

Wilson, E. E-_._. $182-454$

Wilson, J. Tuzo-n..--181-242, 181-248

Wilson, James $\mathrm{T}$

Wilson, R. H. Jr -

Wilson, R. L-_- 182-411

Wilson, W -........ 182-224

Winchester, J. W.......-181-10, 181-11

Windsor, M. W - 180-26

Winkler, H. A-.. 180-155

Winslow, J. D-... 181-471

Witkowski, Jozef -.._-_._- 183-177

Wolf, Helmut-......... 182-422

Wolfe, C. W -

Wolfson, S. H - 182-76

Woodside, W -... 183-389

Woolfson, M. M -............ 183-49

Woollard, G. P-.--181-275, 181-469

$182-83,182-346$

Woolley, W. C - 180-362

World Oil -....... 181-211

Worthing, H. W - 180-17

Worzel, J. L-... 180-191

Wright, C. S-181-376

Wright, L. A-_ 180-12

Wuenschel, P. C - 180-380

Wuerker, R. G -

Wulf, O. R - 180-259

Wyllie, M. R. J - 182-199

Wyllie, P. J - 183-393

Yacob, A - 180-261

Yakovlev, B. M -

Yamaguchi, Rinzo -..-183-159

Yamaguchi, Yushin --.--180-264, 180-265

Yamamoto, M -...- 182-397

Yamasaki, Masao-_-_-_-_-_- 180-409

Yanovskaya, T. B - 181-159

Yanovskiý, B. M -......... 183-421

Yanovskiy, B. U-..--180-29, 180-112 
Abstract

Yarosh, A. Ya -...-183-366, 183-501

Yashchenko, Z. G -....... 183-275

Yasukawa, Katsumi ----1 182-409, 183-452

Yegogyan, V. L - - 183-391

Yekhanan, Ye, V -.....- $182-499$

Yelanskiy, L. N - . 180-269, 182-228

Yelizarova, A. N - 183-11

Yepinat'yeva, A. M - 180-166

Yeremenko, N. A -..... 181-21

Yermakov, V. I -

Yesakov, I. S -

Yokoyama, Hidekichi --- 182-194, 183-230

Yokoyama, Izumi -

Yoshikawa, Soji --_--_ 180-394, 180-408

Young, B. G - $181-335$

Yudin, G. T- 183-258

Yutlandov, I. A -... 180-24

Zablocki, C. J - 183-269

Zaccara, Gaetano -...-. 180-160, 181-281

Zacher, E. G - 183-335

Zadorozhniy, I. K -

Zagmarmistr, A. M --.- 180-106, 181-193

Zägwijn, W. H -

Zähringer, J -....- 181-65, 182-69, 182-75

Zakashanskiy, M. S -.-- 182-430, 182-431

Zandle, G. L -..... 183-478, 183-479 $183-480,183-481$

Zaporozhets, V. M $180-337,181-422$
Abstract

Zátopek, Alois -....-.-182-109, 183-502

Zavelev, A. I --

Zav'yalov, V. D-_.-_ $181-476$

Zeller, E. J -

Zel'tsman, P. A -

Zemtsov, A. A - . 180-238

Zenkovich, V-1..... 180-401

Zharkov, V. N ............. 181-319

Zhirov, K, K -...- 183-32

Zhirova, V. V -... 181-6,181-47, 181-49 $182-44,182-456,183-32$

Zhivago, A. V -

Zhuk, I. Ya -.... 180-377

Zhukhovitskiy, A. A -

Zietz, Isidore -..--.-- 183-465, 183-482

$183-484,183-486$

$183-488,183-490,183-492$

Zijderveld, J. D. A -

Zikmunda, O--.-- 182-502

Zotov, P. P - 183-176

Zounkova, Milada-_-_-_-_ 181-475

Zubov, V. G - 183-196

Zumberge, J. H -

Zutshi, P. K - 181-420

Zverev, S. M -...... 181-456, 182-483

Zybin, K. Yu -

Zykov, S. I -.... 180-5, 180-7, 180-247

$181-5,181-47,181-49$

$182-44,183-32,183-37$ 


\section{SUBJECT INDEX}

A

Accelerograph, optico-mechanical: Korostin 183-172

Acoustic logging, calibration: Schwae tzer 181-450

combination: Burton 182-204

core- and shot-hole loggers: Hardy $182-497$

digital computers: Broding 182-496, 183-560

Oklahoma, Morrow sand: Millard $182-498$

porosity: Berry 180-373, 181-452; Millican 180-375

seismic interpretation: Rimmer 183561

time discrepancies: Gretener 183559

transit-time scale: Widess 183-558

C.S.S. R. : Kozlov 183-577

velocity information: Hardy 181-451

Acoustic waves, in two-component systems: Knopoff 180-241

marine sediments: Shumway 182-482

Aeromagnetic maps: See Magnetic surveys

Africa, age, general listing: Holmes 183-21

age, zircon, Cameroons and French Equatorial Africa: Bessoles 183-24

zircon, Togo and French East Africa: Roques 183-26

explosion seismology, nuclear explosions: Rocard 181-176

praleomagnetism, Late Carboniferous to Late Triassic: Nairn 180289

radioactivity exploration, methods: Bigotte 180-345

seismicity, West African Rift Valley: de Bremaecker 181-98

Age, Antarctica, thermoluminesence: Zeller 182-57

Appalachians: Hurley 181-25

beach ridges, Alaska: Moore 183-19

beaches, Morocco: Gigout 181-43

biotite, Egypt, Dara granite: Hurley 181-44

Ontario: Aldrich 181-35

Scotland: Giletti 180-11
Age-Continued

biotite and feldspar, Ontario: Hurley 181-37

brannerite, Morocco: Ledent 182-45

California, Rancho La Brea: Howard 181-31

Canada, boundary between 1,700 and 2,600 million year provinces: Hurley 181-32

compilation through 1959: Lowdon $182-35$

Chattanooga shale, $\mathrm{U}-\mathrm{Pb}$ determination: Cobb 181-28

crustal adjustments, distribution in time and space: Gastil 180-3

Ellesmere ice shelf, carbon-14 determinations: Crary 182-40

feldspar, Scotland: Giletti 180-11 galena, France: Durand 183-27

general listing, Africa: Holmes 18321; Schürmann 182-46

Antarctica: Starik 183-46

Germany: Schürmann 182-46

Indonesia: Schürmann 182-46

Madagascar: Besairie 183-22

Morocco: Choubert 183-25

glacial deposits, North America: Rubin 182-34

Ontario: de Vries 182-38

glaciation, Antarctica: Péwé 183-47, 48

glauconite, U. S. S. R. : Polevaya 18333; Rubinshteyn, 181-2

gneiss, Brazil: Hurley 181-42

granite, Alps: Jäger 180-13

Antarctica: Cameron 182-56

Australia: Compston 182-55; Fisher 183-42

France: Deutsch 182-42

Germany: Vinogradov 182-44

Nova Scotia: Fairbairn 181-38, 39

Ontario: Wetherill 182-37

Switzerland: Jäger 182-43

U. S. S. R. : Atrashenko $183-40$; Komlev 180-5, 6, 183-38; Rubinshteyn 181-2; Studenikova $180-4$

Gubik formation, Alaska: Coulter 183-18

igneous rocks, France: Pelletier 180-20 
Age-Continued

igneous rocks - continued

Newfoundland: Fairbairn 181-41

U. S. S. R. : Bykovskaya $182-51$; Firsov 182-53; Polevaya 182-52

United States: Jaffe 180-17

igneous and metamorphic rocks, California: Wasserburg 180-12 lead isochrones, age of crust: Baranov 180-248

meteorites: Fesenkov 183-55; Fireman 181-61; Fisher 183-65; Voshage 180-15

Breitscheid: Paneth 183-74

Carbo and Treysa: Voshage 180-15

Casas Grandes and Keen Mountain: Hoffman 182-72

cosmic age: Baranov 181-64; Goebel 181-65; Murin 180-24; Schaeffer $182-73$

I-Xe method: Eberhardt 183-71; Reynolds 183-66, 68; Wasserburg 183-69

lead-thallium determinations: Anders 183-63

rhenium-osmium method: Herr $182-70$

Sikhote-Alin iron: Vinogradov 181 58

mica, Colorado: Giffin 181-29

Georgia: Hurley 181-27

monazite and cheralite, India: Sivaramakrishnan 182-54

Northwest Territories, Ellesmere Island: Blackadar 182-39

peat, New Zealand: McKellar 183-44 pegmatite, India: Aswathanarayana 181-51

U.S. S. R. : Zhirova 181-49

Precambrian, Australia: Hurley 181-52

Baltic shield: Polkanov 183-31

Finland: Simonen 183-30

India: Krishnan 183-41

Manitoba: Moore 181-34

Sweden: Magnusson 183-29

U. S. S. R. : Komlev 183-36; Semenenko 183-34; Vinogradov 183-35; 183-37

radiocarbon dates, Arctic ice islands: Crary 182-40

Arctic Ocean: I. G. Y. Bulletin 180399

Australia: Lundelius 183-43

Canada: McCallum 182-25

Denmark: Krog 183-28; Tauber $182-19$
Age-Continued

radiocarbon dates-continued

Denmark and Netherlands: Andersen 182-41

England: Godwin 182-24

general listings: Barker 182-21; Crane 182-22; Olsson 182-28; Rubin 182-29; Stuiver 182-23; Tauber 182-20

New England and Nova Scotia: Lyon 181-32

New Zealand: Schofield 183-45

Norway: Nydal 182-26

Sweden: Östlund 182-30

United States: Bray 182-25; Hubbs 182-31

Red Sea, latest movements of graben: Nester off 182-47

Rhode Island, Pennsylvanian rocks: Hurley 181-26

schists, South Orkney Islands: Miller 183-20

syenite, Ontario: Fairbairn 182-36

U. S. S. R. : Rubinshteyn 181-2

tektites, potassium-argon: Gentner $182-75$

strontium-rubidium: Pinson 181-73

U.S.S.R. , Aldan shield: Sudovikov $182-50$

Bavly series: Timergazin 181-48

Belomorye complex: Shurkin 18249

Georgian S. S. R. : Rubinshteyn 18145

Karelia: Kratts 182-48

terrestrial deposits, tracing provenance: Krylov 181-50

time scale: Semenenko 182-3

Ukraine S. S. R. : Komlev 180-6; Polevaya $181-46$; Vinogradov 181-47

Urals and Pri-Ural areas: Ovchinnikov 183-39

United States, Early Man: Mason $182-33$

universe, based on gravity: Gilbert $182-281$

uranium deposits, Ontario: Mair 181-36

Pennsylvania: Stern 183-16

vitrain, Nova Scotia: Tupper 181-40

water, U. S. S. R. : Kortsenshteyn 180-22; Mavritskiy 180-21

Wisconsinan stage, Illinois: Frye 181-30

Unites States: Leighton 183-17

wood, Saskatchewan: Kupsch 181-33

U. S. S. R: Vinogradov 181-17 
Age - Continued

zircon, Africa, Cameroons and French Equatorial Africa: Bessoles $183-24$

Africa, Togo and French East Africa: Roques 183-26

Madagascar: Besairie 183-23

See also Age determinations

Age determinations, alpha-counting method: Kapitanov 180-18

argon diffusion: Evernden 183-12; Gerling 183-13

Cambrian System, base of: Davidson 183-4

carbon-14 method: Broecker 180-19; Crevecoeur 182-16; Delaney 181-16; Tamers 182-15; Vinogradov 181-17; Willis 182-17

glauconite, collection of stratigraphically dated specimens: Hurley 181-22

haloes: Deutsch 181-19

helium: Baranovskaya 181-8

helium-argon ratio, oil: Maksimov 181-21

index and bibliography, North America: Gheith 181-23

ionium method: Baranov 181-18

lead, method of extraction: Starik 181-333

lead-alpha (Larsen) method: Gottfried 180-16

lead-210 and lead-212 method: Ledent 180-9

lead isotope method: Catanzaro 1824; Kollar 183-409; Russell 1817; Starik 183-11; Tilton 183-10; Zhirov 183-32

lead-uranium analysis, oscillograph polarography: Zhirova 181-6

meteorites, argon-39: Wänke 182-64 methods, general discussion: Hurley 181-3

reliability of: Cook 183-6

microcline, argon retention: Sardarov 180-14

potassium-argon method:Afanas 'yev 183-14 Firsov 182-8; Hurley 181-12; Krylov 182 -12; Kulp 1811; Marmo 182 -7; Murina 181-13; Rubinshteyn 182-13; Sardarov 181-14; Shukolyukov 181-15; Sudovikov 182-9; Wänke 182-6

potassium-40, flame-photometry analysis: Khanayev 182-11

Precambrian stratigraphy: Quennell 183-3
Age determinations-Continued

radiogenic gases, diffusion in silicates: Amirkhanov 182-10

radium-uranium equilibrium, $\mathrm{sec}-$ ondary minerals: Stern 180-8 rubidium-strontium method: Compston 180-250, 182-55; Crocket 181-10; Fairbairn 181-24; Gerling 180-10; Herzog 181-9, $182-$ 5

samarium-146, possible use in age studies: Nurmia 183-15

sedimentary rocks: Kulp 182-2

separators; biotite, glauconite and muscovite: Rubinshteyn 182-14

thermoluminescence, granitic rocks: Komovskiy 181-20

time scale: Davidson 180-2; Faul 182-1; Follinsbee 183-1; Holmes 180-1; Kulp 181-1, 182-1, 1833; Rubinshteyn 181-2

U.S. S. R. , 1958, status: Starik 181-4 techniques and equipment: Amirkhanov 182-10

United States, review of period 1957 60: Aldrich 183-5

uranium-lead method: Danilevich 183-9; Kuroda 183-7, 8; Tugarinov 181-5; Vinogradov 1807

See also Age

Alaska, age, beach ridges: Moore 183-19

age, Gubik formation: Coulter 183-18 crust, thickness: Woollard 181-275 earth current activity, $1956-58$ reported: Hessler 183-93

earthquakes, 1958: Davis 183-104, 108; Miller 183-105; Stauder 183-107; Tocher 183-103, 106

fault-plane solutions: Stauder 18192

gravity surveys: Oldham 182-310; Woollard 181-275

magnetic field, variations: Alaska University 182-383

magnetic surveys: Zietz 183-492

Alberta, seismic surveys, multiple signals: Magrath 183-570

Algeria, earthquakes: Doubourdieu 181-97

seismic surveys, Hassi Messaoud field: Bouchon 183-573

Alps, age, granitic rocks: Jäger 18013

Amplifiers, geophones: Watt 183-171 
Antarctica, age, general listing: Starik $183-46$

age, glaciation: Péwé $183-47,48$ granites: Cameron $182-56$

thermoluminescence: Zeller 182-57 crust, thickness: Robin 182-279

geophysical surveys: Blackwell 183289; I. G. Y. Bulletin 182-226; Weihaupt 182-230

glaciers, Ross Ice Shelf: Zumberge $183-349$

gravity and seismic surveys: Sorokhtin 182-507

gravity survey: Shumskiy 180-234; Thiel 181-293

ice thickness: Cailleux 182-280; Model' 183-290; Robin 181-480, $182-279$

isostatic adjustment: Ushakov 181 245

isotopes, oxygen in snow: Gonfiantini 180-249

magnetic exploration, features $u$ nique to the Southern Hemisphere: Karasik 182-421

magnetic surveys, bedrock relief: Glebovskiy 181-402

microseisms: MacDowall 180-323

paleomagnetism, Jurassic: Blundell 180-292

Paleozoic and Mesozoic: Bull 181 392

Precambrian: Nagata 180-291, 182416

submarine geology: Lisitzin 181-491

whistlers and chorus: Martin 183-447

Appalachians, magnetic surveys: King $183-484$

seismic surveys: Snow 181-470

Arabian Sea, submarine canyons: Hayter 182-526

Arctic, age, island and shelf ice: Crary 182-40

geophysical surveys, Drifting Station Charlie: Cromie 181-488

Fletcher's Ice Island (T-3): Keller 180-150, 181-212

seismic surveys, Fletcher's Ice Island: Crary 183-572

Arctic Ocean, age, sediment: I. G. Y. Bulletin 180-399

Arkansas, crust, thickness: I. G. Y. Bulletin 182-350

Asia, gravity surveys, Karakorum and Hindu Kush ranges: Desio 183-383
Asia-Continued

seismicity maps: Gorshkov 181-101

Atlantic Coastal Plain, exploration summary: Johnston 182-215

Atlantic Ocean, isotopes, carbon-14: Broecker 183-403

microseisms: Tabulevich 183-503

Mid-Atlantic Ridge, median valley: Hill 182-524

radioactivity: Nelepo $183-517$

sediment cores, magnetic properties: Keen 183-455

shelf, sub-bottom reflections: Ewing $183-583$

Aurora, general discussion: Mariani $182-394$

Australia, age, granites: Compston 182-55: Fisher 183-42

age, Pleistocene faunal succession: Lundelius 183-43

Precambrian: Hurley 181-52

carbon isotopes, Permian coals: Compston 182-369

crust, thickness: Doyle 180-102

earthquakes, relationship to structure: Jones 180-45

electrical surveys, sulfides: Horvath $180-135$

electromagnetic surveys, graphite: Milton 183-251

geophysical surveys, Talisker mine: Milton 182-229

gravity, absolute value at Adelaide: Mumme 182-309

observations: Jackson 181-273

gravity surveys, Cowell area: Seeds man 182-434

Mt. Lofty Ranges: Mumme 182-325

Perth Basin: Thyer 182-326

Rough Range anticline: Dooley 180233

isogonic map, epoch 1960.5: Parkinson 180-255

magnetic exploration, remanent magnetization: Green 181-379

magnetic surveys, Cowell area: Seedsman 182-434

iron deposits: Whitten 182-433

scheelite: Horvath 181-401

sulfides: Smellie 180-316

microseisms, cyclones: Newman 182-442

oil exploration: Bureau of Mineral Resources, Geology and Geophysics 183-288

radioactivity exploration, airborne methods: Mumme 182-466 
Australia-Continued

radioactivity surveys, Olary Province: Harris $180-360$

Radium Hill: Hiern 182-473: Mumme $182-474$

seismic surveys, oil exploration: Harris 180-395

volcanic activity, submarine: Sprigg $181-500$

Austria, earthquakes, 1949-58: Trapp 180-36

seismic surveys: Förtsch 181-474; Janoschek 183-574

Azerbaijan S. S. R. , earthquakes, 1954-56: Sultanov 180-37

Azores, crustal deformation, relation of volcanism: Mendonça Dias 180-402

volcanic eruptions, Fayal: Tazieff $181-496$

B

Baltic Sea, gravity surveys: Honkasalo 181-279

recent deformation: Köster 183-342 Baltic shield, age: Polkanov 183-31 Basin and Range province, crustal structure: Berg 183-395

gravity surveys: Mabey 183-371

Bay of Bengal, submarine canyons: Hayter 182-526

Belgian Congo, gravity and magnetic surveys: Jones 181-278

magnetic surveys: Jones 181-395

Beryllium, quantitative determinations: Vaughn 182-454

Black Sea, relief of sea floor: Lacombe 182-525

Borneo, magnetic survey, basement structure: Agocs 180-313

Brazil, age: Hurley 181-42

geophysical surveys, Amazon Basin: Morales 183-281

British Columbia, gravity surveys, Salmon glacier: Russell 183-375

British Honduras, age, radiocarbon dates: Barker 182-21

Bulgaria, meteorites, Kon'ovo chondrite: Nikolov 181-67

C

California, age, igneous and metamorphic rocks: Wasserburg 180-12
California-Continued

age, Rancho La Brea: Howard 181-31

crust, structure: Press 181-305

crustal deformation: Thompson 183327

earthquakes, 1957: Bonilla 181-88; Cloud 181-90; Hudson 182-86;

Tocher 181-91

1959: Richter 183-101, 102

frequency: Tocher 181-89

San Andreas fault: Oakeshott 181-87 faulting, San Andreas fault: Crowell 183-328

geotectonics, recent deformation: Greene 183-334; Steinbrugge 183-335; Tocher 183-336; Whit ten 182-269, 183-337

gravity surveys, Los Angeles Basin: McCulloh 183-374

Mono Basin: Pakiser 181-274

Mount Whitney: Oliver 183-373

magnetic surveys, Mono Basin: $\mathrm{Pa}-$ kiser 181-274

offshore areas: Bromery 183-489

seismic surveys, offshore areas: Moore 183-584

Canada, age, Blind River uranium deposits: Mair 181-36

age, boundary between 1,700 and 2,600 million year provinces: Hurley 181-32

compilation of data through 1959: Lowdon 182-35

Precambrian: Moore 181-34

radiocarbon dates: McCallum 182 25; Stuiver 182-23

electrical logging, Alberta: McCros san 183-270

electrical surveys, gold: Kelly 180306

lead-zinc: Lundberg 180-124

Noranda Mine, Quebec: Kelly 180126

sulfides: Bergey 180-125; Paterson 181-201

faulting, transcurrent: Haites 183338

geophysical exploration: Latus $182-$ 221; Morley 180-153; Oil in Canada 182-222, 183-280: Oilweek 181-453; Smellie 182-220

geophysical research: Garland $182-$ 237; Hodgson 182-238

geophysical surveys, lead-zinc: Chisholm 180-156

New Brunswick: Ward 180-154 
Canada-Continued

geophysical surveys-continued sulfides: Seigel 180-155

Yukon and Northwest Territories: Campbell 182-225

glaciers, Saskatchewan Glacier: Meier 183-348

gravity surveys, Alaskan Highway: Oldham 182-310

meteor crater near Holleford, Ontario: Bancroft 182-295

North Sturgeon Lake field: Stackler $181-276$

magnetic field, altitudes to $150 \mathrm{~km}$ : Conley 181-345

magnetic surveys, Alberta: Garland $183-476$

asbestos: Conn 180-300; Low 180299

gold: Kelly 180-306

Hudson Bay: Bower 183-498

iron deposits: Fleming 181-394; Ratcliffe 180-305

magnetite: Wahl 180-301

mapping igneous contacts: Koulomzine 180-303

niobium: Westrick 180-304

Prince Edward Island: Canada Geological Survey 183-495

Prince Edward Island and Nova Scotia: Canada Geological Survey $183-496$

sulfide deposits: Bergey 180-125

titanium minerals: Moyd 180-302

paleomagnetism, Precambrian: Du Bois 183-462

seismic surveys, Northwest Territories and Arctic Ocean: Oilweek 183-571

Caribbean Sea, seismic surveys: Officer 180-243; Goedicke 180-388

Chattanooga shale, age: Cobb 181-28

Chile, age, radiocarbon dates: Stuiver $182-23$

crust, thickness: I. G.Y. Bulletin $182-350$

gravity survey, Chillán region: Lomnitz 183-378

volcanoes, maars: Illies 180-405; Miller 183-594

China, geophysical exploration: Jeoujang 181-231; Westgate 183-286

geotectonics: Chin 180-199

meteorites, listing: D'yakonova 181 69

seismicity, Gan'suy Corridor: Petrushevskiy 181-103
China-Continued

seismicity-continued

general characteristics: Mey 182104

magnitude equation: Savarenskiy $182-103$

map: Shan-pan 182-102

Colombia, age, radiocarbon dates: Tauber 182-20

volcanic eruption: Ramirez 180-404

Colorado, age, Precambrian basement: Giffin 181-29

heat flow, borehole measurement: Boldizsár 182-333

Colorado Plateau, electrical exploration, resistivity: Keller 180-122

geophysical surveys, Lisbon Valley area: Byerly 182-218

Compaction, sediments, function of pressure: Parasnis 181-485

Congo, geophysical surveys, Congo basin: Evrard 182-227

Connecticut, gravity surveys: Eaton 183-370

Continental drift, disputed, based on heat flow: Jacobs 181-238

disputed, based on thickness of basaltic layer: Lyustikh 181-244

paleomagnetism, evidence from: Collinson 183-459

Continents, origin, convection currents: Havemann 183-91

Contour maps, trend surface analysis, discussion: Krumbein 181-223

Convection currents, crustal shift: Vening Meinesz 183-330

mantle: Lyustikh 182-259

stepped erosion surfaces: Geyl 182274

Core, composition: Knopoff 180-241, 181-322; Wada 182-367

density transitions: David 182-365 melting point: Strong 182-366

melting point of iron, calculated: Strong 181-294

nutation, action on: Fedorov 181-147

temperature: Gilvarry 181-296; Jacobs 181-295

Cosmogony, passage of stars near the earth, origin of evaporites: $\mathrm{O}-$ mori 182-60

Creep, ice: Mellor 180-215

Crust, basaltic: Hess 182-261

composition, compared with meteoites: Gast 181-316

continental and oceanic rock types: Macdonald 182-348 


\section{Crust-Continued}

deformation, determination by triangulation: Lukásc 181-236

mechanism: Mendonça Dias 180402; Thompson 183-327

energy: Bishopp 182-262; Lyustikh 183-394

heat distribution: Hayakawa 180-235; Jobert 182-330; Wyllie 183-393

local variations, Poisson's ratio: Kamitsuki 180-59

melting temperatures: Wyllie 183393

methods of study:Afanas'yev 182-345 phase relations of minerals: Kennedy 182-342

review of research 1957-60: Ewing $182-347$

secular movements, U. S. S. R. : Meshcheryakov 182-271

strength, analysis: Carey 182-514

stresses, Pacific Ocean: Balakina 180-47

structure, Alaska: Woollard 181-275

Basin and Range Province: Berg 183-395

California-Nevada region: Press 181-305

Caribbean: Officer 180-243

Germany: Closs 182-355

Hawaii: Shor 183-396

North America, east coast: Drake 180-202

North and South America: I. G. Y. Bulletin 182-350

Pacific Ocean: Utsu 182-357

Puget Sound area:Neumann 182-351

South Africa: Gane 182-353; Hales 182-352

U. S. S. R. : Gal'perin 182-356; Godin 183-576; Moiseyenko 183398; Veytsman 182-344

thickness, Antarctica: Robin 182279

Australia: Doyle 180-102

Eurasian continent: Porkka 183399

Finland: Penttilä 183-397

relationship to gravity: Koryakin 181-306

seismic refraction measurements: Mason 182-349

tilt: Dubrovskiy 180-51; Tomaschek 180-221

Czechoslovakia, gravity network, correction: Burša 182-303

gravity surveys, vertical gravity calibration lines: Chudoba 182315
Crust-Continued

gravity surveys - continued

Vienna basin: Beránek 182-320; Doležal 182-316; Ibrmajer 182$317,318,319$

magnetic field, epoch 1952.5: Ochaba 181-399

epoch 1958: Boushka 182-381

secular variation: Bucha 181-350 magnetic field base network, linked with Poland: Bucha 182-380 magnetic surveys, Bohemian batholith: Běhounek 181-398

Prúbram: Krs 181-397

Sumperk area: Jelen 182-426

seismic exploration, air-shooting: Beránek 181-447

seismic surveys, Little Danubian Plain: Beránek 181-475

Vienna basin: Beránek 182-320

seismicity, Komarno region: Kárnîk 182-92

D

Demagnetization, marls: Creer 180282

Denmark, age, radiocarbon dates: Andersen 182-41; Krog 183-28; Tauber 182-19, 20

Density log, review: Pickell 182-458 Rocky Mountain area: Edwards 180339

Diastrophism, types: Billings 181-237

Diffusion-adsorption potentials, cause, Hittorf number:

Vendel'shteyn 181-187

Dipmeter logs, electronic analog computer: Braun 182-236

E

Earth, figure, computation center at Ohio State University: Uotila 183-312

figure, departure from exact spheroid: Bomford 181-233

determination: Arnold 183-313; Fischer 180-188; Plakhov 180189

equation for rigorous determination: Arnold 182-248

geodetic and astronomic determination: Burš 182-252

gravity determination: Boaga 182249; Uotila 182-246

historical review: Johns 182-245

lunar parallax method: Plakhov 1.80-190

Molodenskiy's method: Arnold 183313 
Earth-Continued

figure -continued

north-south asymmetry: Carey 180-184; O'Keefe 180-184

satellite observations: Arnold 180181; Cohen 183-315; I. G. Y. Bul letin 180-185

general nature, review: Stumpff 183298

interior, electrical properties: Tozer 181-207

general discussion: Gutenberg 181 303

methods of study:Jeffreys 182-341; Woollard 182-346

petrochemical interpretations: Fischer 182-340

phase relations of minerals: Kennedy $182-342$

physical state: Lucke 182-343

review of research 1957-60: Birch $182-338$

temperature: Verhoogen 182-328

textbook: Trevisan 181-225

origin, cold: Shmidt 181-304

rotation, effect of core: Brower 18075

effect of magnetic storms: Danjon $181-375$

effect of solar activity: Bernard 181-146

measurement of variations: Markowitz 181-149; Stoyko 181-148

variation related to deep earthquakes: Stovas 180-52

variation related to movement of poles: Melchior 180-74

thermal history: Lyubimova 181-297

Earth currents, Alaska, 1956-58 activity reported: Hessler 183-93

convective current: Izergin 183-95

diurnal disturbance, U.S.S. R. : Mishin 181-83

exploration, U.S.S.R. : Nikitenko 183-96

geomagnetic disturbance: Hessler 182-81; Miguel y Gonzales Miranda 182-402

Germany:Repsold 180-30; Schmuck er 181-180, 182-82

instrumentation, filtering slow variations: Dupuy 180-31

micropulsations, equator: Hutton $182-384$

model study, induced currents: Volland 181-77

medium of variable conductivity: Bossy 181-79
Earth currents-Continued

nomogram, vertorial diagrams: Popov 181-84

topographic and geologic effect:

Wescott 183-94

variation: Niblett 183-92; Verö 181 81; Vladimir ov 181-82; Yamaguchi 180-265

vector diagrams, use in mining districts: Porstendorfer 181-78

Earth tides, acceleration, sun and moon effect: Longman 180-71

geotectonic energy: Zotov 183-176

gravity corrections: Service Hydrographique de la Marine and Companie Générale de Géophysique 180-72

harmonic analysis: Pertsev 180-70; Pícha 182-148

measurements: Longman 183-175; Slichter 182-146; Witkowski 183-177

perturbations: Jobert 182-147

pole tides: Haubrich 180-73

Earthquakes, aftershocks: Senshu $182-116$

Alaska, 1958: Davis 183-104, 108; Miller 183-105; Stauder 183-107; Tocher 183-103, 106

Algeria: Doubourdieu 181-97

Austria, 1949-58: Trapp 180-36

Azerbaijan S. S. R. , 1954-56: Sultanov 180-37

California, 1957: Bonilla 181-88; Cloud 181-90; Hudson 182-86; Tocher 181-91

1959: Richter 183-101, 102

frequency related to major earthquakes: Tocher 181-89

San Andreas fault: Oakeshott 18187

China, seismicity map: Shan-pan $182-102$

deep-focus: Stovas 180-52

dislocation theory: Droste 181-114

Dominica, West Indies, unidentified tremors: Robson 183-110

effects on buildings: Bonelli Rubio 182-123; Bycroft 183-146, 147; Medvedev 180-69

effects on dams: Sinitsin 183-148

E1 Salvador, 1959: Boletin, Sismológico del Servício Geológica Nacional de El Salvador 183-109 energy: Gutenberg 182-106; Solov'yev 181-105

epicenters: Golenetskiy 183-135; Pachadzhanova 180-66 
Earthquakes-Continued

fault-plane solutions, Alaska: Stauder 181-92

geometric study: Nagumo 181-116 technique: Båth 181-113; Vvedenskaya 183-142

U. S. S. R. : Keylis-Borok 182-120; Scheidegger 182-121

focal mechanism: Aki 180-55

focus, determination of strain at: Shirokova 181-115

relation to structure: Kukhtikova 180-39

tsunami determinations: Savarenskiy $180-48$

Hawaii, 1958: Eaton 180-35

horizontal movement: Whitten 183144

intensity, abnormal distribution in Japan: Wadati 182-105

analysis: Neumann 182-110

Russian scale: Medvedev 182-111 Japan, 1958: Quarterly Journal Seismology 182-88

landslides: Glukhov 182-124

location of, computer method: Flinn 183-134

magnitude, calculations for Collmberg seismic station: Panner 182-113

calculations for Rome seismic station: Di Filippo 181-106

determination: Bisztricsány 181104, 182-107; Kárnîk 182-109 Solov'yev 181-105

function of azimuth: Kurimoto 181109

relation to number: Tsuboi 181-108

Rumania: Iosif 181-107

U.S.S. R. : Savarenskiy 182-114; Shebalin 182-108

mechanism, first motions:

Knopoff 181-112

formation of joints: MacCarthy $182-118$

large-scale: Komura 182-119

microearthquakes:Mikumo 180-54

quadrant-type initial motion: $\mathrm{Ka}-$ mitsuki 180-60

Rayleigh and Love waves used in analysis: Aki 181-111

S-wave analysis: Stauder 183-138, 140

theoretical analysis: Byerly 181 110; Chinnery 183-137; Droste 180-53
Earthquakes - Continued

Mongolia, macroseismic observations: Ninzhbadgar 182-101

Montana, 1959: de Costa 182-84; Jackson 183-99; Matthews 18186; Nuttli 183-100; O'Reilly 180-34; Stewart 183-98

Morocco, 1960: Gorung 183-111; Tillotson 181-93

origin: Balakina 180-47; Petrushevskiy $183-143$

prediction, seismoelectric phenomena: Dubrovskiy 180-51

tilt observations: Arkhangel'skiy 180-65; Karmaleyeva 183-145

relationship to structure, Australia: Jones 180-45

source functions, obtained from Rayleigh waves: Aki 182-117

submarine landslides, tsunami produced by: Popov 180-49

surface loading: Ambraseys 180-68 temporal distribution:Shlanger 183136

tsunami, Greece: Ambraseys 181-94

U.S.S.R. , Azerbaijan: Sultanova $182-95$

Baku, 1958: Gorin 181-96; Kuznetsov 181-95

Gobi, 1957: Pasechnik 180-44; Solonenko 183-113

Kamchatka: Stauder 183-139; Benioff 180-61

Kurile-Kamchatka zone: Kondorskaya 182-93

magnitude and energy: Solov'yev $182-112$

North Caucasus: Nikitin 183-118

seismicity atlas: Savarenskiy 181 99

Tadzhik S. S. R., 1949: Leonov 182-87

1956: Gayskiy 180-38; Gubin 18041; Kon'kov 180-42

catalog: Semenov 180-40

Tien Shan, map of epicenters: Vvedenskaya $182-98$

Ulugchat, 1955: Leonov 180-43

United States, 1957 summary: Brazee 180-33

1958: Brazee 183-97

Earthquake waves: See Seismic Waves Egypt, age, Dara granite: Hurley 18144

age, Precambrian and Miocene: Schürmann 182-46 
Egypt-Continued

age-continued

radiocarbon dates: Barker 182-21

electrical surveys, Wadi El Kharruba: Ghazarian 182-192

radioactivity, air: El Nadi 183-518

Elastic properties, cores, anomalous velocities: Baule 183-192

damping of waves, specimens of different shape: Shamina 180-92

heterogeneous media: Khaykovich 180-85

homogeneous medium, buried source problem: Mitra 183-186

material under pressure: Vinogradov 180-90

minerals: Verma 181-161

perforated material, seismic modeling: Gil'bershteyn 182-165

peridotite: Hess 182-261

rocks: Kostantinova 180-91; Riznichenko 183-193; Volarovich $181-168,183-195$

rutile, corrected constants: Birch 183-198

sandstones: Mann 181-162

sea ice: Anderson 183-197; Ishida 180-93, 94

sediments: Shumway 181-165

See also Elastic waves,propagation

Elastic waves, anisotropic medium: Duda 183-181

Cenomanian-Senonian stage, England: Laughton 181-166

compressional: Spencer 181-151

determination of pressure at wave front: Bagdoyev 183-183

diffraction: Ben-Menahem 181-121

fluids overlying elastic half space: Strick 180-88

free periods of tortional oscillation: Sato 182-15a

generation, small explosions: Kasahara 183-210

granular rocks:Balakrishna 182-170

ice: Hunkins 183-196

impulse attenuation: Shamina 181170

long-time response, layered elastic medium: Rosenbaum 181-152

longitudinal head waves: Davydova 181-167

Love: Knopoff 180-87; Sato 182-160

maximum vertical displacement: Willis 183-205

model study, dispersion: Sato 182166
Elastic waves-Continued

model study-continued

electric grids: Ivakin 181-172

seismoscope:Riznichenko 181-171

vertical discontinuity: Kun 182-

156; Pariyskaya 183-184

nuclear explosions: Adams 182-175;

Diment 183-199;Rocard 181-176

P-waves: Usami 183-188

plane compressional Voigt: Collins $182-150$

plane transverse polarized: Khaykovich 183-185

pressure component: Nagumo 182173

propagation, bounded solids: Davies 180-83

dual integral transformation method: Nagumo 183-187

elastic cylinder with infinite length: Adachi 182-157

imperfectly elastic medium: Hervas Burgos 182-155

inhomogeneous medium: Richter 180-81

liquid-filled porous solid:Deresie wicz 183-178

multilayered medium: Black 183180; Pod "yapol'skiy 181-156

single layer medium: Knopoff 181 155

solids: Band 181-163

PS converted waves: Schwind 183204

Rayleigh, particle amplitude profiles: Dorman 183-189

recording, galvanometer: Adachi 183-213

reflected, solid-fluid boundary: Oguchi 181-160

refracted, layer of finite thickness: Press 181-154

scattering, by small inhomogeneities: Miles 182-154

shear and compressional: White 182 152

shock, attenuation: Grine 182-153

small-amplitude stress: Knopoff 182-162

spherical source: Honda 181-158

stratified half space: Spencer 182158

stratified medium: Pod"yapol'skiy 181-157

surface, classification: Sato 180-63

synthesis by Fourier transform: Sato 183-190 
Elastic waves-Continued

surface-continued

two types demonstrated mathematically: Perri 182-161

transition layers: Bortfeld 183-179

two-dimensional truncated wedge: Ambraseys 181-150

variations, deformation under high pressure: Matsushima 182-171

velocity: Myachkin 183-194;Nabeoka 182-176; Subbarao 181-164;

Szemeredy 182-169

Elasticity,dynamical problems, Cagniard's method: Mitra 183-182

model study, seismological problems: Hayakawa 182-164

variable velocity depth and density functions: Healy 183-191

semispace, mathematical analysis: Borodachev 180-82

strain energy: Fogelson 182-168

visco-elastic material: Bland 182151

Young's modulus, proportional to resistivity: Yashchenko 183-275

Electrical exploration, anisotropic media: Chetayev 182-180

boreholes: Kozyrin 183-239

boundary value problem: Kolbenheyer 183-217

conductivity as a function only of depth: Belluigi 181-177

cylindrical inclusion, homogeneous isotropic half space: D'yakonov 183-226

diffusion-adsorption potentials:Vendel'shteyn 181-187, 183-218

dipole sounding:Bordovskiy 181-190; Gel'fand 183-228; Krayev 183248; Petr 182-178; Zagarmistr 180-106

direct current, ring array: Shabanov $183-241$

earth currents, U.S.S. R. : Nikitenko 183-96; Vladimirov 181-192; Zagarmistr 181-193

electrical sounding, description of method: Fritsch 182-187

generalized Cagniard graph: Koefoed 183-215

improvement of interpretation: Ono $182-183$

special case: Metzger 182-179

field of frame and cable: Gel'fand 183-227

geoelectric model graphs: Geier 181-185
Electrical exploration-Continued ground resistivity, depth determinations: Kataoka 182-182

ground testers: Vogler 181-196

ice, effect of surface melting on resistivity: Cagniard 182-188

induction, airborne: Tikhonov 181191

dip and strike determination: Rodionov 180-116

instrumentation: Enenshteyn 181195

magnetic field, induced at earth's surface: Stefănescu 181-189

model study, application: Utzmann 180-104

boundary values of triaxial ellipsoid: Kolbenheyer 181-186

polarized conductors: Gruntorad $181-188$

ring induction integrator: $\mathrm{Al}$ 'pin 183-229

multilayer resistivity problem:

Onodera 183-214

natural field: Novozhilova 180-117; Semenov 180-120

nonconducting strata, shielding effect: Tikhonov 181-179

overvoltage: Brant 182-184

plane problems: Glyuzman 183-233

point source, anisotropic space: Semenov 182-181

polarization, field equipment desscribed: Nosske 182-195

membrane potential: Kudymov 183219

potential fields, classification of anomalies: Tyapkin 183-220

profiling methods: Gruntorad 181194; Kruszewski 183-231

ratio of potential gradients method: Zavelev 183-243

resistivity, archeological exploration: Diceglie 182-191

Colorado Plateau: Keller 180-122

discussion: Seigel 180-119

methods applicable in valleys: Dizioğlu 182-189

uranium exploration: Keller 180123

self-inductance, straight grounded wire: Overholt 183-216

self-potential: Kelly 180-105; Ohashi 181-198; Shibato 183-244

sensitivity to inhomogeneities: $\mathrm{Sa}-$ kovtsev 1/3-234 
Electrical exploration-Continued

sphere near contact with two media: Matveyev 181-180

structural regionalization: Fomina $183-242$

vertical depth profiling, interpretation: Dzhafarov 180-109, 183238

vertical electrical sounding: Dorofeyev 183-237, 240; Kozyrin 183-236; Sakovtsev 183-235

voltage control, automatic compensator: Mizyuk 182-196

Electrical logging, apparent resistivity microsondes: Per'kov 182 207

carbonate reservoirs: Molochnikov 181-206

combination: Doll 182-200; Pomerants 183-264

dip determinations: Swift 180-141

electrodes, resistance: Polyakov 181-205

electroseismic effect, permeability determination: Kindij 180-366

field interpretation: Alger 182-203 formation factor: Fujiwara 183-263 FoRxo log: Towle 181-203

fundamentals: Biggs 182-202

Guard log: Towle 181-203

induction: Aksel'rod 183-265; Kaufman 183-261; Nikitina 183-256

interpretation, sandstone reservoirs: Wyllie 182-199

Texas Gulf coast region: Walker 181-202

laterolog: Al'pin 183-260; Chukin 183-257; Itenberg 183-272; Lehnert 181-204; Melik-Shakhnazarov 183-266

$\log$ interpretation charts: Schlumberger Well Surveying Corporation 183-253

magnetic disturbances: Garland 182 198

microlog: Leont'ev 180-139; Holgate $182-460$

physical properties of rocks: Kobranova 183-308

porosity, use in calculation of reserves: Kotyakhov 182-209

porosity structure: Buryakovskiy $183-262$

radioactivity logging, use with: Becker 180-157

resistivity, effect of dissolved salts: noc $182-205$
Electrical logging-Continued

resistivity-continued infiltration zone: Kulinkovich 180137

lateralog: Kukharenko 180-138

mapping reefs in Alberta:McCros san 180-143

reservoir properties determined from: Moroz ov 180-136

slide rule: Roeschmann 182-197

U. S. S. R. : Grechukhin 180-146

review of late developments:Mathieu $183-252$

self-potential: Safontsev 183-258; Segesman 182-201

sonde to recorder communication: Voskoboynik 180-142

steaming potential: Bernstein 180140

sulfide ores: Anderson 183-267

surveys, Canada: McCrossan 183270

Mississippi: Lang 182-211

Missouri: Zablocki 183-269

Tennessee: Keller 183-268

U.S.S. R. : Chernyshev 180-144; Dolitskiy 180-145; Itenberg 183271; Umantsev 182-210

transition zone in oil pools, model study: Sokhranov 183-259

translations from English to Russian: Dakhnov 183-255

water wells, specific capacity analysis: Bennett 183-254

Electrical potential, scalar: Belluigi 180-103

Electrical properties, earth's interior: Tozer 181-207

frozen ground: Cook 182-213; Hatherton 183-273

piezoelectric effect:Volarovich 181208

resistivity, high confining pressure: Volarovich 183-274

sandstones, Morrison formation: Keller 180-123

sulfide ores: Anderson 183-267

Young's modulus, proportional to resistivity:Yashchenko 183-275

Electrical surveys, Australia, sulfides: Horvath 180-135

Canada, gold: Kelly 180-306

lead-zinc: Lundberg 180-124

Noranda Mine, Quebec: Kelly 180126

sulfides:Bergey 180-125; Paterson 181-201 
Electrical surveys-Continued

Egypt, Wadi El Kharruba: Ghazarian $182-192$

France, uranium deposits: Sarcia $183-246$

Germany, electromagnetic: Nosske $181-200$

India, sulfides: Rao 180-129

Jamaica, hydrogeology:Vincenz 180127

Japan, copper: Murozumi 180-133 copper, lead and zinc: Shibato 180118; Yokoyama 182-194

gold: Suyama 180-130 ground water: Homma 183-249 lead and zinc: Odani 180-132 pyrite: Research Group for Spontaneous Polarization Method [Japan] 183-250; Suyama 180131,134

Missouri, ground water: Meidav 183245

Spain, Murcia: Sell Cantalapiedra 183-247

Switzerland, Eau Morte:Poldini 182190

Tanganyika, gold: King 180-308

Tasmania, Renison Bell tin field: Davidson 180-319

Turkey, Azdavay Carboniferous area: Dizioğlu 182-189

U. S. S. R. , ground water: Shayins'kyy 182-193

Electromagnetic exploration, airborne: Lundberg 182 186; $\mathrm{Pa}$ terson 183-223; Ward 180-121

conducting bodies: Wait 182-177, 183-221

cylindrical inhomogeneity: D'yakonov 183-225

dipole sounding:Gasanenko 181-184; Van'yan 180-108

dipping ore vein: Nikitina 183-224

field analysis: Tikhonov 180-110

glaciated areas, slingram method:

Frischknecht 183-222

ground methods: Ward 180-115

ice thickness: Gintzburg 183-232

inverse problem, mathematical treatment: Chetayev 181-181

methods, review: Roux 182-185

Scandinavia: Frischknecht 180-128

U. S. S. R. : Tikhonov 181-197

model study, airborne survey: Hedstrom 180-113

characteristics independent of shape: Svetov 181-182
Electromagnetic explorationContinued

model study, low frequency: Molochnov 180-112

two-coil method: Kunori 183-230

Electromagnetic field, anomalies, triboelectric effect from seismic waves: Parkhomenko 181 169

vertical magnetic dipole over horizontal layers:Gasanenko 181183

Electromagnetic surveys, Australia, graphite: Milton 183-251

Electromagnetic waves, diffraction, circular cylinder in homogeneous half space: D'yakonov 181 178

diffraction, sphere in semispace: D'yakonov 180-111

El Salvador, earthquakes, 1959: Boletin Sismológico del Servício Geológica Nacional de El Salvador 183-109

England, age, radiocarbon dates: Godwin 182-24

elastic waves, Cenomanian-Senonian stage: Laughton 181-166

paleomagnetism, Cretaceous and Tertiary: Wilson 182-411

Jurassic: Girdler 180-283

Late Paleozoic: Creer 180-281

Eniwetok Atoll, drilling operations: Ladd 183-291

Eötvős balance: Silva 182-305

Eurasian continent, crust, thickness: Porkka 183-399

Europe, magnetic field, secular variation: Bock 180-253

microseisms, 1957-58:Zátopek 183502

paleomagnetism, Paleozoic, Mesozoic, and Tertiary: Nairn 181386

Exploration, helium, Saskatchewan: Bateman 182-223;Sawatzky 182 224

submarine gas seeps, method: Dunlap 180-173

Explosion seismology, crustal structure, Australia: Doyle 180-102; Ulomov 181-478

crustal structure, Basin and Range province: Berg 183-395

Germany: Closs 182-355

North and South America: I. G. Y. Bulletin 182-350 
Explosion seismology-Continued ground waves: Berg 180-97; Carder 180-95; Martin 183-207; O'Brien 181-174; Willis 183-205

hypocenter determinations: Asano 180-101

India: Tandon 183-209

Japan:Research Group for Explosion Seismology [Japan] 180-99

long-period waves: Oliver 182-174 mechanism: Van̋̌ 180-98

mine collapses: Sponheuer 183-208 nuclear explosions, 1957-58 test series: Diment 183-199

Africa 1960:Rocard 181-175, 176 fracturing: Shoemaker 183-202; McKeown 183-200; Wilmarth 183-201

PS converted waves: Schwind 183204

seismic waves: Pomeroy 183-203 wave amplitudes: Romney 180-96 recording instrument: Alekseyev $181-465$

seismic waves, distinguished from explosion waves: Kogan 181-128

S-waves: Asano 180-100

timing equipment: Riihimaa 183-211 wave attenuation: Iida 180-368

\section{F}

Faulting, Africa, Western Rift Valley: Berg 180-203

California, San Andreas fault: Crowell 183-328

mechanism: Jobert 183-141

Mexico, Agua Blanca fault of Baja California: Allen 181-241

transcurrent: Haites 183-338; Lensen 180-201; Schofield 183-323

Fiji Islands, thermal springs: Healy $183-600$

Finland, age, Precambrian rocks: Simonen 183-30

crust, thickness: Penttilä 183-397 recent deformation: Honkasalo 183341

seismicity: Penttilä 183-115

Florida, magnetic surveys: King 180198

Folding, mechanism: Belousov 183318; Bemmelen 183-329; Khain 183-319

Fracturing, brittle material: Lisowski 180-89
Fracturing-Continued

Griffith theory, extension: Brace $183-579$

France, age, galena: Durand 183-27

age, granite: Deutsch 182-42

lava flows: Pelletier 180-20

electrical surveys, uranium deposits: Sarcia 183-246

geophysical surveys, canal engineering: Mouton 182-240

oil: Gangloff 180-149

Roussillon basin: Gottis 181-213 gravity, absolute value at Sèvres: Thulin 182-308

magnetic surveys, Jura Mountains: Schwab 181-396

paleomagnetism, Cenozoic: Glangeaud 181-387

Jurassic: Girdler 180-283

Miocene: Roche 182-412

radioactivity surveys, Mortagne granite: Roubault 183-509

radon in natural waters: Jurain $183-515$

Frictional properties, joints: Jaeger 180-398

\section{G}

Geodesy, altitude of observation points: Hirvonen 183-311

base lines: Heiskanen 181-232

basic hypothesis, check by satellite data: O'Keefe 180-183

beach marks, marine: Ewing 180-191 datums: Iz otov 180-193; Meade 180192

deflection of vertical: Chovitz 180187

dictionary, Polish-Russian-German-English-French: Sztompke 181-235

dynamic system: Braaten 180-194 gravity anomalies; isostasy: Kivioja $181-251$

gravity reductions: Rice 180-186

Hiran network: Corpacius 183-316 hypsographic curve, isostatic adjustment: Lagrula 181-234

latitude determination, Denmark: Poder 183-317

measurements: Cook 180-176

planet triaxial ellipsoidal form: Boaga 182-253

reference ellipsoid, U. S. S. R. : Izotov 180-193 
Geodesy-Continued

satellites: Thomas 182-254

textbook: Burkard 182-256

Geoid, shape: Heiskanen 183-310; Uotila 182-247; Veis 182-255

world ellipsoid: Fischer 182-250

Geomagnetic depth profiling, Germany: Schmucker 181-80

Geomagnetic dipole, relation to dip pole: Parkinson 181-348

Geomagnetic tides, India: Rao 180262

L currents: Rao 180-260

Geophones, amplifiers: Watt 183-171 borehole: Dennison 181-445

evolution of types:Richards 181-459

Geophysical exploration, activity in 1959: Patrick 183-276

Antarctica:Blackwell 183-289;I.G.Y. Bulletin 182-226; Robin 181-480

Atlantic Coastal Plain:Johnston 182215

Australia and New Guinea: Bureau of Mineral Resources, Geology and Geophysics 183-288

Canada, 1959: Latus 182-221; Oil in Canada 180-152, 182-222

1960: Oil in Canada 183-280

airborne: Oilweek 181-453

evaluation: Nielsen 180-148

petroleum: Westgate 183-286

current problems:Dennison 181-209

damsites: Masuda 183-287

France, canal engineering: Mouton $182-240$

Great Britain: Taylor 183-299

industry and mining: Meisser 183301

Italy, archeology: Lerici 181-214 summary: Beneo 182-313

textbook: Aquilina 181-226

Nordic Meeting of Mining Geophysics, 1959: Saxov 183-300

oil, need for direct methods: Roy 183-278

operations research: Cobb 183-297 Philippine Islands, airborne: Rankin 181-222

salt domes: Halbouty 182-216

statistical approach in planning: Slichter 183-279

techniques, developments in 1959: Engineering and Mining Journal 182-239

textbook: Dobrin 182-232; Russell 182-233

U. S. S. R. , 1959-65: Geologiya Nefti $181-228$
Geophysical exploration-Continued

U.S.S.R. , cost analysis: Kozlov 181-217

gold: Safronov 182-243

Kuybyshev Trans-Volga area: Yelanskiy 182-228

methods: Andreyev 182-242; Burtmar 181-219

organization: Kozlenko 181-218

summary: Sungurov 180-162

Ural-Volga area: Godin 181-215

United States, decline in activity: World Oil 181-211

drilling in 1959: Blanpied 183-277

wildcat wells: Blanpied 182-217

Geophysical quantities, controlled by sun, effect of time: Dominici 183-296

Geophysical research, Canada, review for 1957-60: Hodgson 182238

Canada, review for 1959: Garland $182-237$

correlation methods:McDonald 183295

harmonic analysis of data: Sugiura $183-441$

I. G. Y. , general results: Chapman 180-174

logging data, advantage over laboratory data: McCollum 180-171

mathematics used in: Baranov 183293; Longman 183-292

summaries of progress: White 180147

U. S. S. R. , evaluation: Khitarov 181 229

Geophysical surveys, Antarctica, ice thickness: Model' 183-290

Antarctica, Victoria Land traverse: Weihaupt 182-230

Arctic, Drifting Station Charlie: Cromie 181-488

Fletcher's Ice Island (T-3): Keller 180-150, 181-212

Australia, Talisker mine: Milton $182-229$

Brazil, Amazon Basin: Morales 183-281

Canada, New Brunswick:Morley 180153; Ward 180-154

review of 1959: Smellie 182-220 sulfides: Seigel 180-155

Yukon Territory:Chisholm 180-156

Yukon and Northwest Territories: Campbell 182-225

China, 1949-59: Jeou-jang 181-231 status: Yepinat'yeva 180-166 
Geophysical surveys-Continued

Colorado Plateau, Lisbon Valley area: Byerly 182-218

computers, application:Assiter 181 210

Congo, Congo basin: Evrard 182-227

France, Roussillon basin: Gottis $181-213$

uranium exploration:Gangloff $180-$ 149

Germany, joint systems of mineral springs: Nosske 183-283

western Bavaria: Breyer 183-282

Greenland, 1959-60: Finsterwalder 180-213

Hungary, oil: Oszlaczky 180-158 Italy, Apulia region: Zaccara 180160

Japan, lead-zinc: Odani 180-169 uranium:Kobayashi 180-167; Odani 180-168

New Zealand, Taupo-Tarawera district: Modriniak 180-170

Poland, northeast: Skorupa 183-284

U. S. S. R. , 1955-58: Godin 182-241

Astrakhan: Rakitov 180-164

Caspian Sea: Mustafayev 183-285

Dnieper-Donets depression: Andreyeva 181-221

evaluation: Brod 181-216

Georgian S.S.R. : Balavadze 181 307

Lower Volga area: Kozlenko 181 220

Siberia: Savinskiy 181-227

Turkmen A.S. S. R. : Kasatkin 181 230

Washington, Hanford Works: Brown 183-309.

Wyoming, Horse Creek field: Peters 182-219

Geophysical well logging, calculation of reserves: Kotyakhov 182-209

carbonate reservoirs: Chombart $183-305$

coordinator in operating company: Johnson 182-234

Hungary: Bélteky 180-159

logging programs: Kirby 180-172 methods:Dakhnov 182-208; Doll 183304; Mathieu 183-252

parameters of rocks: Itenberg 183306

Pennsylvania, catalog: Wagner 180151

production logging: Bryant 182-235

progress, 1955-59: Doll 183-303
Geophysical well logging - Continued sonic and resistivity combination: Burton 182-204

U.S.S.R., Neftyanyye Kamni: Buryakovskiy 180-165

oil-gas reservoirs: Dakhnov 182206

Utah, Paradox Basin: Millard 181 224

Venezuela, porosity determinations: Haeberle 182-212

Geophysics, handbook: Geophysics Research Directorate 183-294

Georgia, age, micas: Hurley 181-27

Geotectonics, arcs or alpinotype: Hess $182-261$

basins: Fairbridge 182-264

continental growth: Wilson 181-242

continents and oceans, development: Kraus 180-198

crustal shift: Vening Meinesz 183330

energy source, earth tides: Zotov $183-176$

expanding earth: Beck 181-239; Carey 180-184; Egyed 182-267, 325, 339; O'Keefe 180-184; Wilson 181-248

folding: Belousov 183-318

geologic cycle: Harpum 183-326

geosynclines, mechanism: Belousov 181-243

isostatic compensation, origin of continents: Griggs 182-258

lateral thrust: Wolfe 183-322

model studies, imbrication folding:

Köster 183-347

review:Gzovskiy 181 -249

supplement to field studies: Belousov 183-346

North Carolina, coastal plain block tectonics: Ferenczi 182-268

Pacific basin, rotation: Biq 182-273 phase transitions: MacDonald 182257

phases, nature and duration: Bubnov 180-200

processes, China: Chin 180-199

recent deformation, Asia: Ma 183333

Baltic Sea: Köster 183-342

California: Greene 183-334; Steinbrugge 183-335; Tocher 183336; Whitten 182-269, 183-337 correlation with Bouguer anomalies: Donabedov 183-358

Finland: Honkasalo 183-341 
Geotectonics-Continued

recent deformation, Italy: Salvioni $182-270$

Japan: Hatai 183-345

Mexico: Allen 181-241

Red Sea graben: Voûte 183-340 removal of ice: Ushakov 183-344 Rumania: Ez 181-246

Spitsbergen: Birkenmajer 183-343 U.S.S. R. :Meshcheryak ov 182-271 regional structural analysis:Azhgirey 183-320

spherical harmonics, orogenic implications:Vening Meinesz 180197

Taiwan, place in circumpacific tectonics: Biq 183-331

tensions, generated by unequal heat distribution: Jobert 182-330

Western.Pacific: Ehara 182-272

Geothermal energy, Iceland: Mérial 182-541

Italy: Mériel 182-541

Kenya: McCall 183-598

Mexico: de Anda 183-597

New Zealand: Mériel 182-541

Geothermal gradient, determination: Kraskovskiy 181-298

effect of magma: Hayakawa 180-235 effect on mine workings: Kappelmeyer 180-237

oil pools: Ramazanzade 180-390

U.S.S. R., Armenian S.S. R. : Ananyan 183-391

West Siberian Lowland: Koshlyak 182-336; Ryabukhin 182-337

Geothermal heat, Japan: Hayakawa 180-235

Geothermal zoning, U.S.S. R., West Siberian Lowland: Mavritskiy 181-299

Geothermometry, quartz, veins in basalt: Savul 181-302

Germany, age, granites: Schürmann 182-46; Vinogradov 182-44

crustal structure: Closs 182-355

earthquakes, magnitude equation for Collmberg seismic station:

Panner 182-113

electrical exploration, vein deposits : Nosske 181-200

geomagnetic depth profiling: Schmucker 181-80

geophysical surveys, joint systems of mineral springs: Nosske 183283

western Bavaria: Breyer 183-282
Germany-Continued

gravity surveys,Erzgebirge:Grosse $181-280$

Halle Market Place Fault: Hohl 183-379

heat flow: Schössler 183-386

magnetic surveys, Eifel: Cipa 180310

Saxony: Neumann 182-425

paleomagnetism, Permian: Schmucker 180-286

radioactivity, thermal spring deposits at Baden-Baden: Kirchheimer 183-510

seismic surveys: Andres 182-503; Thomas 183-575

Geysers, mechanism: Golovina 183596

Glaciation, cause, multiple factors: Emiliani 180-204

cause, water vapor variation: Gosselink 180-205

Glaciers, Antarctica: Robin 181-480; Zumberge 183-349

Canada, Saskatchewan Glacier: Meier 183-348

crystal orientation, natural and artifically deformed ice: Rigsby $182-275$

Greenland, review article: Fristrup 182-278

movement:Griffiths 182-276; Meier 183-348; Merrill 183-350; Nye 180-207; Wallerstein 180-209

Novaya Zemlya: Svatkov 183-351 physical properties, measurements in tunnels: Butkovich 182-277

seismic surveys, Austria: Förisch $181-474$

stress: Nye 180-206

thickness, Antarctic icecap: Cailleux 182-280; Robin 182-279

velocity:Mathews 180-214; Nye 180206

Gravimeters, airborne: Thompson 181-268

calibration: Gilbert 181-266

marine:Harrison 182 -301; Lozinskaya 180-225; Popov 181-270;

Sukhodol'skiy 181-269

Nörgaard: Burša 182-303; Dimitrov 180-223

pressure changes, effects of: Gan$\operatorname{tar} 181-267$

Russian Sn-3: Dimitrov 180-223

zero-point creep: Popov 182-306

Gravity, absolute value, Adelaide, Australia: Mumme 182-309 
Gravity-Continued

absolute value, Sèvres, France:

Thulin 182-308

Bouguer anomalies: Donabedov 183358; Galushko 182-289

calotte correction, Cassinis' numbers as weights: Norinelli 182285

Czechoslovakia, base network: Burša $182-303$

deflection of the vertical:Arnold 182288

density determinations: Egyed 181259

determination above earth's surface: Arnold 182-283

digital computing methods:Bott 181 265

earth's shape, methods of determination: Uotila 182-246

elliptical paraboloid, gravity poten tial of: Gavrilov 182-284

Eötvōs balance: Silva 182-305

equipotential surfaces, density discontinuities: Olberg 181-252

formulas, Somigliana's: Boaga 180177

free-air reduction: Arnold 182-251

free fall of graduated scale:

Thulin 182-308

geoid shape, determination: Uotila 182-247

higher derivatives: Beránek 182-291

Hungary, base netowrk: Renner 182 322

maps, analysis: Matsuda 180-218

error estimation: Sazhina 181-263

material half plane: Gladkiy 183-356

observations, Australia:Jackson 181-273

satellites: Cook 182-287

South Africa: Gough 181-272

pendulum apparatus, improved bronze type:Thompson 182-302

pendulum measurements, North Central, and South America: Jackson 181-271

planet, triaxial ellipsoidal form: Boaga 182-253

reduction, improvement of accuracy: Raspopov 180-216

rock density: Jung 181-253

secular variations: Barta 182-307 sphere and cylinder, nomograms for calculating effect of: Válek 182290

statistical and harmonic analysis: Kaula 180-182

\section{Gravity-Continued}

theory of relativity: Gilbert 182-281

tide effect: Service Hydrographique de la Marine and Companie

Générale de Géophysique 18072

topographic-isostatic reduction maps, Europe: Heiskanen 182312

variation, atmospheric pressure effect: Tomaschek 180-221

vertical distribution, compared with horizontal distribution:Tomoda 181-257

Gravity anomalies, analysis, mass distribution underground: Tomoda 181-258

classification, basis: Balabushevich 180-219

computation, above surface: Tsuboi $182-299$

any elevation by Fourier series: Tsuboi 181-250

higher elevations: Tsuboi 183-353 crustal thickness, linear relationship: Koryakin 181-306

geodetic application, isostasy: $\mathrm{Ki}-$ vioja 181-251

interpretation: Klushin 183-357; Mangadze 183-355

modelling, optical: Roy 180-220

regional gravity field, differentiation from: Fajklewicz 182-296

three-dimensional bodies: Talwani 180-217

Gravity exploration, accuracy of parameters: Pola 182-294

airborne gravimeter: Nettleton 180224

anomalies, smoothing along traverses: Malovichko 183-361

closure of network:Butakov 182-297

correlation of adjacent areas: Jones 181-262

elevation, photogrammetric determination: Kinoshita 181-260

exchange center: Bike 183-302

gravity difference between stations: Murphy 183-368

horizontal gravity gradient: Thyssen-Bornemisza 183-367

interpretation, case of two density discontinuities: Pola 182-292

isopach residuals:Stackler 181-276

iron ores: Hinze 181-264

lignite: Yarosh 183-366

logistical and field methods:Nettleton 183-376 
Gravity exploration-Continued magnetic data, use in gravity calculations: Tyapkin 183-360

magnetic exploration, combineduse of methods: Karatayev 183-359 methods, description: Pemberton 180-222

photogeology, use in interpretation: Whitworth 181-261

reduction, mountainous areas: Adzhimamudov 183-362

reduction to Bouguer plate:Pola 182 293

running average method: Seya 182300

temperature effect: Faytel'son 183 369

terrain correction: Kane 183-363

two-dimensional body: Tyapkin 181255

U. S. S. R., density data: Oganisyan 181-291

review: Uspenskiy 182-298

ultramafic rocks: Davis 183-364

upward continuation of data, relaxation method: Roy 183-354

vertical component, infinite horizontal cylinder: Caputo 181-254

Gravity field, check by satellite data: O'Keefe 180-183

distribution of continents, inconsistancy with nonhydrostatic harmonics: Munk 182-282

external: Cook 180-179; Orlin 180180; Vening Meinesz 180-178

two dimensional, linear transformation: Kalinina 181-256

vertical gradient: Kantas 182-286 Gravity logging, density determinations: Veselov 180-226

vertical gradient: Egyed 183-365

Gravity potential, second, fourth, and sixth harmonics: King-Hele $183-314$

Gravity surveys, Alaska, crustal thickness: Woollard 181-275

Antarctica, nature of continent: Shumskiy 180-234

tide determinations: Thiel 181-293

U. S. S. R. expedition; Sorokhtin 182-507

Asia, Karakorum and Hindu Kush: Desio 183-383

Australia, Cowell area: Seedsman $182-434$

Mt. Lofty Ranges: Mumme 182-325

Perth Basin: Thyer 182-326
Gravity surveys-Continued

Australia-continued

Rough Range anticline:Dooley 180233

Baltic Sea: Honkasalo 181-279

Basin and Range province: Mabey 183-371

Belgian Congo: Jones 181-278

California, Los Angeles Basin: McCulloh 183-374

Mono Basin: Pakiser 181-274

Mount Whitney: Oliver 183-373

Canada, meteor crater near Holleford, Ontario:Bancroft 182-295

North Sturgeon Lake field: Stackler 181-276

Salmon glacier: Russell 183-375

Canada and Alaska, Alaskan Highway: Oldham 182-310

Chile, Chillán region: Lomnitz 183378

Connecticut: Eaton 183-370

Czechoslovakia: Beránek 182-320; Chudoba 182-315; Doležal 182316; Ibrmajer 182-317,318, 319

Germany:Grosse 181-280; Hohl 183379

Greenland: Svejgaard 181-277

Hungary: Renner 181-282; Szilárd $182-321$

India: Caputo 181-254

Italy: Beneo 182-313; Salvioni 182314; Zaccara 181-281

Japan: Otaki 183-384; Yokoyama 183-385

Nevada Test Site: Diment 183-372

New Zealand: Robertson 182-327

Paradox basin: Joesting 183-487

Rumania: Airinei 181-283, 182-323; Botezatu 181-284, 286; Stoenescu 180-228, 229, 181-285

South Africa: Hales 182-352

Switzerland: Poldini 182-190

U. S. S. R. , Armenia: Oganisyan 181 290, 182-324

Bashkir and Tatar A. S. S. R. : Volodarskiy 180-231

Caspian area and Ustyurt: Nevolin 181-288

Emba region: Kotlyarevskiy 181289

Kalmyk A. S. S. R. :Ostrovskiy 180163

Kuban River and Caspian Sea areas : Dzhafarov 183-380

Minusin trough: Tarkov 180-230

proposed network: Grushinskiy 181-287 
Gravity surveys-Continued

U. S. S. R. , Rudnyy Altay and Kalba regions:Gorzhevskiy 181-292

Siberian platform: Vasil'yev 180232

Tatar A. S. S. R. : Boronin 183-381: Salikhov 183-382

West Siberian Lowland: Agul' nuk 182-430; Zakashanskiy $182-431$

Union of South Africa, gold: Weiss 180-307

United States, activities of 1957-59: U. S. Coast and Geodetic Survey 182-244

Venezuela:Hospers 182-311, 183-377

Great Britain, age, radiocarbon dates: Barker 182-21

Greece, earthquakes, tsunami: Ambraseys 181-94

Greenland, geophysical surveys, 195960: Finsterwalder 180-213

glaciers: Fristrup 182-278; Griffiths 182-276; Wallerstein 180-209

gravity surveys: Svejgaard 181-277

ice tunnel, deformation: Hilty 183581

isotopes, deuterium and oxygen-18 in ice: Dansgaard 181-327

radioactivity, Skaergaard pluton: Hamilton 181-410

seismic surveys, Thule area:Roethlisberger 182-502

thermoluminescence, limestones: Zeller 182-18

Ground water, carbon-14 as tracer: Fergusson 182-449

Guatemala, volcanoes: Williams 183589

$\mathrm{H}$

Hawaii, crust, structure:Shor 183-396 earthquakes, 1958: Eaton 180-35 seismic surveys: Shor 183-396 volcanic activity, Iki crater 1959: Simpich 181-495

Iki crater 1959-60:Bellair 181-494 Iki crater 1960: Shale Shaker 183592

Kilauea: Friedman 182-527

volcanoes, growth: Eaton 183-588

phreatomagmatic explosions: Fraser $183-587$

Heat, conductivity: Jaeger 180-236; Parasnis 181-485; Ratcliffe 181-301; Woodside 183-389

distribution, crust: Jobert 182-330
Heat flow, borehole measurements: Boldizsar 182-333; Glyuzman 183-388

continental drift: Jacobs 181-238

core, melting point of iron: Strong 181-294

temperature: Jacobs 181-295

crust, melting temperatures in:

Wyllie 183-393

Germany: Schössler 183-386

Hungary, measurements: Stegena 182-334

Nagylengyel oil field: Boldizsár 182-335

instrumentation, recording from a ship: Babenkov 183-392

layer of finite thickness, during cooling: Glyuzman 183-387

mantle, melting point: Valle 182-375 metallogenesis, role in: Smyslov 182-331

mine workings: Kappelmeyer 180237

permafrost, construction problems: Khaskind 180-239

principles reviewed: Schössler 183386

sources: Verhoogen 182-328

thermal history of earth:Lyubimova 181-297

thermistors, diamond: Electronics 182-332

topography effect: Jobert 182-329

Hungary, earth currents: Verö 181-81

geophysical surveys:Oszlaczky 180158

geophysical well logging: Bélteky 180-159

gravity surveys: Renner 181-282, 182-322; Szilárd 182-321

heat flow: Boldizsár 182-335; Stegena 182-334

I

Ice, deformation: Butkovich 180-208; Landauer 180-210, 211; Rausch 180-212; Rigsby 182-275

physical properties, strain gauges: Clark 182-516

Iceland, geothermal energy: Mériel 182-541; Vlodavets 181-497

paleomagnetism, Pliocene-Pleistocene: Rutten 183-463

seismic exploration: Bath 182-354

volcanoes: Thorarinsson 182-529; Vlodavets 181-497 
Illinois, age, Wisconsinan stage: Frye $181-30$

India, age, monazite and cheralite: Sivaramakrishnan 182-54

age, pegmatite: Aswathanarayana $181-51$

Precambrian rocks: Krishnan 18341

radiocarbon dates: Barker 182-21

electrical surveys: Rao 180-129 explosion seismology: Tandon 183209

geomagnetic tides: Rao 180-262

gravity survey, Godwin Austen glacier: Caputo 181-254

magnetic field: Yacob 180-261

magnetic surveys, manganese deposits: Rao 180-312, 182-405

microseisms, Bay of Bengal: Iyer $183-505$

radioactivity, air: Lal 181-420

seismic waves, velocity in limestone: Quadir 181-479

Indian Ocean, submarine geology: Ewing 181-490

Indiana, seismic surveys: Rudman 183-568

Indonesia, age, Mesozoic tin province: Schürmann 182-46

Induced polarization, cause, osmotic pressure: Rokityanskiy 180-107

Iran, age, radiocarbon dates: Barker 182-21: Crane 182-22

seismicity, map: Kirillova 183-122

Ireland, paleomagnetism, Cretaceous and Tertiary: Wilson 182-411

Isogonic map, Australia and New Guinea, epoch 1960.5: Parkinson 180-255

Isostasy, Antarctica: Ushakov 181245

crustal recovery, due to removal of ice: Ushakov 183-344

crustal thickness, Union of South Africa: Gel'fand 180-227

depth of compensation: Nanda 180242

gravity anomalies, geodetic application: Kivioja 181-251

North America, oscillatory adjustment: Sargent 183-339

reduction maps, Europe: Heiskanen $182-312$

Isotopes, aluminum-26, meteorites and tektites: Anders 182-66
Isotopes-Continued

argon, meteorites: Fireman 181-61, 183-62; Schaeffer 183-61; Signer 183-60; Vinogradov 181-58

potassium minerals: Gerling 181331

beryllium-7, rainwater: Rama 183408

carbon, Atlantic Ocean: Broecker $183-403$

concentration in carbon cycle: Broecker 183-404

ground-water tracer: Fergusson $182-449$

nuclear explosions: Latter 181-416, Plesset 181-415

Permian coals of Australia: Compston 182-369

produced by solar protons: Simpson 181-325

cosmic-ray produced, tracers in atmosphere: Peters 181-417

deuterium, Greenland ice:Dansgaard 181-327

deuterium and tritium, nuclear explosions: Begemann 181-323

fractionation, sulfur: DeChow 181330

helium, meteorites: Hoffman 180-

23, 182-72; Paneth 183-74;

Schaeffer 183-61; Signer 18?-60; Vinogradov 181-58

helium and argon production, iron targets: Schaeffer 182-69

hydrogen, Antarctic snows: Picciotto $183-402$

krypton, uranium minerals: Young $181-335$

lead, age of the earth's crust: Baranov 180-248

Blind River uranium deposits: Mair 181-36

dating galena: Russell 181-7

extraction method: Starik 181-333

leaching: Naydenov 183-410

U. S. S. R. : Vinogradov 180-247

lead-210, decay constant: Eckelmann 183-506

meteorites, oxygen fractionation: Vinogradov 181-60

neon, meteorites: Schaeffer 183-61; Signer 183-60; Vinogradov 181 58

origin: Kashkarov 183-508

noble gases, meteorites: Fechtig 181-57 
Isotopes - Continued

oceans: Broecker 182-371

origin, stellar evolution: Cameron 180-245

oxygen, Antarctic snows: Gonfiantini 180-249

Arctic foraminifera: Ault 181-326 method of determination: Dontsova $183-405$

oxygen-18, Greenland ice:Dansgaard 181-327

Philippine Trench: Dansgaard 183406

oxygen-18/oxygen-16 ratio, paleotemperature measurements: Tudge 181-328

phosphorus-32, rainwater: Rama $183-408$

radioactivity logging: Blankov $181-$ 424

rare earths, cosmic origin: Taylor 183-411

research in United States, 1957-59: Hoering 183-401

silicon-32, marine environment: Lal $181-329$

strontium, granite: Compston 180250

distribution in United States:Skougstadt $182-370$

strontium-87, variations in crust and mantle: Hurley 181-332

sulfur: DeChow 181-330; Gavelin 183407; Östlund 182 -372; Rama 183408; Vinogradov 180-251

thallium, magmatic rocks: Golebchina 180-252

thallium-105, meteorites: Anders 183-63

tritium, meteorites: Fireman 181 61, 62; Goebel 183-64

nuclear spallation: Singer 182-373 origin: Wils on 181-324

solar protons: Simpson 181-325 uranium, relative abundance: Hamer $183-412$

xenon, atmosphere: Kuroda 183-70 xenon-129, meteorites: Eberhardt 183-71; Reynolds 181-334, 183$66,67,68$; Signer 183-72; Wasserburg 183-69

uranium minerals: Young 181-335 Italy, geophysical exploration: Aquilina 181-226; Lerici 181-214 geophysical surveys: Beneo 182-313; Zaccara 180-160
Italy-Continued

geothermal energy: Mériel 182-541 gravity surveys: Beneo 182 -313; Salvioni 182-314; Zaccara 181-281 radioactivity, Biella granite-syenite: Fiorentini-Pontenza $182-445$

recent movements: Salvioni 182-270 seismicity: Malaroda 182-91

volcanoes, Stromboli: Cavallero $181-498$

\section{$\mathrm{J}$}

Jamaica, electrical surveys: Vincenz 180-127

radioactivity, mineral spring: Vincenz 181-412

seismic stations: Robson 183-174

Japan, age, radiocarbon dates: Stuiver $182-23$

earthquakes, 1958:Quarterly Journal Seismology 182-88

intensity: Wadati 182-105

elasticity, model: Hayakawa 182-164 electrical surveys, copper: Murozumi 180-133

copper, lead, zinc: Shibato 180-118; Yokoyama 182-194

gold: Suyama 180-130

ground water: Homma 183-249

lead-zinc deposits: Odani 180-132

pyrite: Research Group for Spontaneous Polarization Method [Japan] 183-250; Suyama 180131,134

explosion seismology, Kamaisi mine: Research Group for Explosion Seismology [Japan] 18099

geophysical surveys, lead-zinc deposits: Odani 180-169

uranium: Kobayashi 180-167; Odani 180-168

geotectonics, recent deformation: Hatai 183-345

geothermal heat: Hayakawa 180-235

gravity surveys: Otaki 183-384; Yokoyama 183-385

magnetic field: Kato 182-399; Rikitake 180-266

mantle: Kishimoto $182-364$

microseisms: Okano 182-441

orogenesis: Ehara 183-332

paleomagnetism, Cretaceous and Tertiary: Nagata 180-290, 181 391 
Japan-Continued

radioactivity, atmosphere: Lockhart 180-331

feldspar and biotite:Hayase 182-446 granites: Tsutsumi 182-447

Ogamo and Ningyotoge mines: Hayase $182-448$

radioactivity exploration, airborne methods: Iwasaki 180-348

radioactivity surveys:Horikawa 180 355, 357; Iwasaki 180-349, 350, 353, 358; Kaneko 180-352, Kinosaki 182-472; Matsubara 182469; Nakai 180-356, 359; Niino 183-513; Owa 182-468; Sugiyama 180-351; Tokunaga 182-470; Ujiie 180-354; Ukai 182-471

seismic surveys, fault zones: Kaneko 183-578

seismic waves, $L_{\mathrm{g}}$ type: Utsu 182357

velocities: Yoshikawa 180-394

thermal springs, Joban coal field: Nakamura 181-501

Onikobe Basin: Nakamura 182-544

regional properties: Nakamura 180-413

Sambei volcanic area: Ando 182545

volcanic activity, Aso Volcano: Matsumoto 182-535; Namba 182534; Yoshikawa 180-408

prediction: Minakami 182-539

submarine explosions: Morimoto $183-595$

volcanoes, Mt.Asama:Sekiya 182-536

relation to faults: Sato 181-499

Joints, frictional properties, measurements: Jaeger 180-398

\section{K}

Kenya, geothermal energy: McCall 183-598

L

Latitude, determination of, Denmark: Poder 183-317

Least-squares method, variant:Stearn 183-231

Leveling, altitude variation: Németh 180-195

Louisiana, seismic surveys, helicopter transport: Conselman 181-467
Love waves, long period, dispersion at surface: Jobert 182-130

spherical heterogeneous earth model: Sato 182-159, 160

\section{M}

Maars, Chile: Illies 180-405; Miller 183-594

Madagascar, age, general listing: Besairie 183-22

age, zircon: Besairie 183-23

Magnetic anomalies, classification: Balabushevich 180-219

interpretation: Allingham 183-485; D'yachkov 183-475; Henderson 182-417; Strakhov 183-470, 473 optical modeling: Roy 180-220

significance, moderately deformed sediments: Grantz 183-490

Magnetic bays, auroras, common cause: Bless 181-358

Magnetic declination, Czechoslovakia, tables for 1850-1950: Svoboda 181-344

Magnetic exploration, Antarctica: Karasik 182-421

calibration of instruments: Hoylman 180-293

compass and magnet: Wahl 180-296

dip needle: Westrick 180-297

dikelike bodies: Andreasen 183-465

exchange center: Bike 183-302

geological mapping: Stam 183-467

gravity calculations: Tyapkin 183360

gravity exploration, combined: Karatayev 183-359

magnetometers: Scott 180-294; Smellie 180-295

micromagnetic: Kašpar 182-418

ore deposits: Wahl 183-468

radiogeodetic adjustment: Lozinskaya $183-474$

regional structure sections: Mikhaylov $182-420$

remanent magnetization: Girdler $183-469$

selection of methods: Paarma 183466

shape of body: Kazinskiy 181-393

two dimensional bodies: Polonskiy $182-419$

upward continuation of data: Roy 183354 
Magnetic exploration-Continued variometers: South African Mining Engineering Journal 182-423

weak alternating fields: Berkman 183-472

Magnetic field of the earth, atmospheric ozone: Vassy 183-449

aurora: Mariani 182-394

base networks, Czechoslovakia and Poland linked: Bucha 182-380

cause: Bailey 183-416; Cowling 183418; Herzenberg 183-417

sum of two dipoles: Lyakhov 183415

$\mathrm{C}_{i}$, daily normals: Shapiro 183-438 cosmic-ray equator:Pomerants 183423

cosmic rays : Kodama $183-427$

Czechoslovakia, map for epoch 1958: Boushka 182-381

disturbances: Cole 182-374; Ellis 181-367; Hill 183-442: Mariana 183-448; Pohrte 183-439; Uyeda $180-263$

dynamo theory: Takeuchi 181-338

earth currents, exploration: Schmucker 182-82

electrical logging: Garland 182-198 external, measurements: Dolginov 181-346; Sonett 183-425; Vestine 181-336; Wilson 181-347

fluctuations: Duffus 181-376; Lowrie 181-368; Onwumechilli 182-385; Wilcox 183-446

general discussion: Thellier 181-339 geomagnetic dipole: Parkinson 181348

horizontal component: Kalashnikov $183-429$

hydromagnetic waves: Akasofu 183435

induction coil, study of rapid variations: Miguel y Gonzales Miranda 182-402

ionospheric sounding: Ose 183-450 measurement of, Rumania: Milea 180-256

South Africa: Heirtzler 183-422

medium of variable conductivity, model study: Bossy 181-79

micropulsations: Afasofu 181-357; Benoit 182-387; Bryunelli 183421; Campbell 182-389

motion, magnitude: McCulloh 181374
Magnetic field of the earth-Continued normal field, derivation: Kautzleben $182-379,183-419$

observatories, Australia: Dooley 180-254

automatic: Alldredge 183-420

program during 1957-60, United States: Nels on $182-378$

pulsations: Campbell 181-359, 360; Halenka 182-391; Hutton 182384; Jacobs 183-436; Kalashnikov 181-373; Obayashi 181-372;

Scholte 181-371

relativity effect: Aslanyan 183-414 reversal: Metallova 180-270

rotation of earth: Bernard 181-146 secular variation: Barta 181-349;

Bucha 181-350; Gall 183-426;

Komarov 183 -428; Kopecký 182 382; Thellier 180-287

solar corpuscular radiation: Beard $183-433$

solar gas: Singer 182-390

solar wind: Beard 183-413; Sonett $183-434$

$\mathrm{S}_{\mathrm{q}}$ field, tidal theory: Ingraham 181352

$S_{\mathrm{q}}$ variation: Kazmi 182-388; Matsushita 183-437

two dimensional, linear transformation: Kaninina 181-256

Van Allen belt, geomagnetic variations: Akasofu 181-354; Dessler 181-337; Parker 183-432

variations, Alaska: Alaska University 182-383

altitude to $150 \mathrm{~km}$ : Conley 181-345 earth currents: Bryunelli 180-29 equatorial ring current: Kertz 181351

Europe: Bock 180-253

focus of geomagnetic $\mathrm{S}_{\mathrm{q}}$ current: Hasegawa 181-353

$\mathrm{H}$ and $\mathrm{Z}$ near equatorial electrojet: Onwumechilli 183-424

India: Yacob 180-261

Japan: Kato 182 -399; Rikitake 180266

longitude effect on horizontal component: Heisler 183-431

Iuni-solar at Ibadan(Nigeria): Onwumechilli 182-386

meteor showers: Jenkins 181-377 period range 0. 3-120 sec: Benioff 181-370

solar eclipse: Yamaguchi 180-264, 265 
Magnetic field of the earth-Continued variations - continued

solar flares: Warwick 181-361

three-dimensional considerations: Fukushima 182-377

universal-time component: Nicholson 180-259

westward drift: Rochester 183-430 vector of magnetic induction: $\mathrm{Kol}-$ benheyer 182-376

whistlers: Kimpara 181-369; Martin 183-447

Magnetic storms, cause: Barsukov 181-362; Danjon 181-375; Dessler 180-257

cosmic-ray events: Bachelet 182395

current systems during: Duncan 183443

Doppler effect: Sugiura 183-441

equatorial regions: Maeda 182-397 general discussion: Mariani 182-393 hydromagnetic theory: Dessler 180257; Francis 181-356; Kato 181364

Mar. 31-Apr. 3, 1960: Chinburg $182-400$

precursory signals: Cecchini 182401

review:Chapman 183-444; Matsushita 182-396

ring-cur rent theory: Hines 180-258; $181-355$

rise times: Dessler 183-440

solar filaments: Bednarova-Novakova 182-392

solar flare of June 1, 1960: Ellison 183-445

sudden commencements: Abe 181366; Burkard 181-365; Matsushita $181-363$; Srinivasamurthy 182-398

Magnetic properties, anisotropy: Howell 183-461; Stacey 182-403, $183-454$

chromite: Jenness 180-276

ilmenite-hematite: Ishikawa 180-274 iron deposits, U. S. S. R. : Arutyunyan 183-456

$\mathrm{FeTiO}_{3}-\mathrm{Fe}_{2} \mathrm{O}_{3}:$ Ishikawa 180-271, 273

magnetostrictive effects: Stott 182407

manganese minerals: Rao 182-405

$\mathrm{NiTiO}_{3}-\mathrm{Fe}_{2} \mathrm{O}_{3}:$ Ishikawa 180-272
Magnetic properties-Continued

paleomagnetism loss with time: Yelanskiy 180-269

pyrrhotite: Saito 180-275

quartz dolerite: Powell 183-453

sediment cores: Keen 183-455

titanomagnetite: Akimoto 180-280

Magnetic recorder, time-sifter type: Frost 181-464

Magnetic surveys, Alaska, petroleum: Zietz 183-492

Antarctica, bedrock relief: Glebovskiy 181-402

Appalachian basin: King 183-484

Australia, Cowell area: Seedsman 182-434

iron deposits: Whitten 182-433

remanent magnetization: Green 181-379

scheelite: Horvath 181-401

scheelite-magnetite deposits: Horvath 180-317

sulfides: Smellie 180-316

Belgian Congo: Jones 181-278, 395

California: Bromery 183-489; Pakiser 181-274

Canada, Alberta: Garland 183-476 asbestos: Conn 180-300; Low 180299

gold: Kelly 180-306

Hudson Bay: Bower 183-498

igneous contacts: Koulomzine 180303

iron: Fleming 181-394; Ratcliffe 180-305

magnetite: Wahl 180-301

niobium: Westrick 180-304

sulfide deposits: Bergey 180-125

titanium minerals: Moyd 180-302

Canada and Alaska, Alaskan Highway: Oldham 182-310

Czechoslovakia, Bohemian batholith: Běhounek 181-398

epoch 1952-5: Ochaba 181-399

Pŕ́bram: Krs 181-397

Sumperk area: Jelen 182-426

England-Lebanon profile, basement structure: Agocs 183-499

Florida: King 180-298

France, Jura Mountains: Schwab 181-396

Germany, Eifel: Cipa 180-310

Saxony: Neumann 182-425

India, manganese : Rao 180-312, 182 405 
Magnetic surveys-Continued

Maine: Allingham 183-477

Malaya: Agocs 180-314, 315

Montana, 10N pluton: Zietz 183-488

New Brunswick, maps: Canada Geological Survey 183-493

New Jersey, maps: Bromery 183480,481

New Mexico, basement configuration: Andreasen 183-486

New Zealand: Studt 180-318

North Carolina: Johnson 183-530

Nova Scotia, maps: Canada Geological Survey 183-494, 496

Scotian Shelf off Halifax: Macpherson 183-569

Ontario, Blind River basin: Steenland 182-424

maps: Canada Geological Survey 183-497

Oregon: Balsley 183-491

Pacific Ocean: Agocs 180-313

Paradox basin, basement structure: Joesting 183-487

Pennsylvania, maps: Bromery 183$478,479,480,481,483$

Triassic structure: Zietz 183-482

Prince Edward Island, maps: Canada Geological Survey 183-495, 496

Rumania: Airinei 180-311, 181-283; Stoenescu 180-228

Spain: Gaibar-Puertas 180-309

Tanganyika: King 180-308

Tasmania: Davidson 180-319

U.S.S.R., Chernigov magnetic anomaly: Andreyeva 183-500

iron ores: Arutyunyan 182-427

Klyuchevskaya volcano:Bernshteyn $182-432$

Kursk magnetic anomaly: Lapina 183-471; Shneyerson 181-400

Tatar A. S. S. R. : Boronin 183-381; Stepanov 182-428

Vyatka and Kama River basins: Yarosh 183-501

West Siberian Lowland: Agul'nuk 182-430; Pudovkin 182-429;

Zakashanskiy 182-431

Union of South Africa:Weiss 180-307

United States, during 1957-60: Nelson 182-378

Washington: Washington Division Mines and Geology:183-531

Magnetic susceptibility, anisotropy, Adirondack granites: Balsley 181-378
Magnetic susceptibility-Continued cleavage in slates: Fuller 182-404 orthopyroxene: Akimoto 180-279 volcanic rocks, New Mexico: Wargo 181-381

Magnetization, instability in rocks: Akimoto 183-451

remanent, basic rocks:Angenheister 180-268

chemically generated: Kobayashi 180-278

instrumentation: Dianov-Klokov 181-382

thermoremanent, origin: Verhoogen 180-267

Magnetohydromagnetic waves: Valle 182-362

Magnetometer, measurement of weak ly magnetized rocks: DianovKlokov 181-382; Hellbardt 180277

microvariation: Bryunelli 183-421

nuclear resonance:Hurwitz 181-341; Shapiro 181-340; Wolf 182-422

Maine, magnetic surveys: Allingham 183-477

Malaya, age, radiocarbon dates: Barker 182-21

magnetic surveys: Agocs 180-314; 315

radioactive survey: Agocs 180-314, 315

Mantle, asthenosphere: Shebalin 182 108; Vesanen 182-361

composition: Gast 181-316

convection cells: Bernshteyn 181 315; Vening Meinesz 180-197

convection currents: Licht 181-317; Lyustikh 182-259

density transitions: David 182-365 discontinuities: Dachille 181-313

low velocity layer: Lyubimova 181 318; Scheidegger 181-247

melting point: Valle $182-375$

olivine-spinel transition: Ringwood $183-400$

periclase in D-layer: Wada 182-368 physical nature: Magnitskiy 181-321

Rayleigh-wave dispersion: Press 181-315

structure: Kishimoto 182-364; Lehmann 182-363:Vvedenskaya 181320

temperature: Gilvarry 181-296

thermal conductivity: Lyubimova 181-297 
Mantle-Continued

thermodynamic analysis: Zharkov 181-319

wave velocities: Gutenberg 180-56

Mars, crustal structure, compared with Earth and Moon: Bülow 183-51

Mediterranean Sea, relief, sea floor: Lacombe 182-525

Meteorites, achondrites: Kvaska 18025

age, argon method: Fireman 181-61; Wänke 182-64

based on nuclear reactions:Fisher 183-65

Breitscheid: Paneth 183-74

calculation: Voshage 180-15

Carbo and Treysa:Voshage 180-15

Casas Grandes and Keen Mountain: Hoffman 182-72

cosmic: Baranov 181-64; Goebel 181-65: Murin 180-24; Schaeffer 182-73

I-Xe method: Eberhardt 183-71; Reynolds 183-66, 68, Wasserburg 183-69

lead-thallium: Anders 183-63

rhenium-osmium: Herr 182-70

tritium content: Goebel 183-64

types of: Fesenkov 183-55

alkali metal content: Gast 181-55 aluminum-26 content:Anders 182-66 argon-37, -39 , and tritium content; Fireman 183-62

carbonaceous chondrites: Vdovykin 183-59

China, listing: D'yakonova 181-69 chondrites: Gast 181-316; Mason 181-53; Ringwood 181-56

collection, Museo de la Plata, Argentina: Radice 183-56

composition: D'yakonova 183-58

cosmic-ray effects: Nier 182-63

cosmic ray produced isotopes: Schaeffer 183-61

cosmic-ray produced rare gases: 183-60

cryptovolcanic structures: Dietz 182-68

deep-sea sediment: Hunter 181-70

density and mass distribution: Brown $182-61$

diamond-bearing: Ringwood 183-54 earth's crust: Vinogradov 181-66 former environment: Goles 183-73 helium and argon production, iron targets: Schaeffer 182-69

inert gases: Fechtig 181-57
Meteorites-Continued

iron: Hoffman 180-23; Levskiy 18265

isotopic fractionation, oxygen: Vinogradov 181-60

isotopes, Sikhote-Alin: Vinogradov 181-58

Kon'ovo chondrite, Bulgaria:Nikolov 181-67

mineral separation: Kirova 181-59

origin: Levin 181-54; Mason 183-52

penetration mechanism: Shoemaker 183-76

physical properties:Alekseyeva 18375

South Africa, Barkly West: Groeneveld 182-74

specific gravity:Pokshivnitskiy $183-$ 53

spherules: Crozier 183-57

stone, cosmic-ray ages: Eberhardt $182-71$

thermal gradient during fall: Maringer 182-67

tritium, production cross section: Singer 182-373

U.S.S. R. , Manych chondrite: Gnilovskoy 181-68

uranium: König 181-63; Starik 18162

velerite: Vdovykin 183-59

xenon-129: Reynolds 181-334; 18367; Signer 183-72

Meteors, origin in comets: Gallagher 182-62

Mexico, age, radiocarbon dates: Crane $182-22$

crust, thickness: I. G. Y. Bulletin $182-350$

geothermal energy: de Anda 183-597

Microseisms, Antarctica: MacDowall 180-323

Atlantic and Pacific Oceans: Tabulevich 183-503

Australia: Newman 182-442

Central Europe, 1957-58: Zátopek $183-502$

classification of submarine strata: Malurkar 182-443

cold fronts, evidence of: Saha 183504

direction of approach:Hollinderbaumer $182-439$

earth rotation: Bernard 181-146

exploration: Due Rojo 182-437

India: Iyer 183-505

Japan: Okano 182-441

origin, oriented winds: Nanda 181 403 
Microseisms - Continued

origin, standing waves: Hieblot 182 436

review of research 1957-60, United States: Carder 182-435

storm location, U.S.S.R. : Monakhov $182-440$

types: Gutenberg 180-320; Strobach $181-405$

U. S. S. R. , Caspian Sea: Tabulevich 180-322

Far Eastern Region: Bukhteyev $181-404$

wave character: Schneider 182-438

weak ground motion: Rocard 182-444

Mid-Atlantic Ridge, median valley: Hill 182-524

Mine collapse, seismic effect: Sponheuer 183-208

Mississippi, electrical logging: Lang $182-211$

Missouri, electrical logging: Zablocki 183-269

electrical surveys: Meidav 183-245 magnetic exploration: Allingham $183-485$

Mohole project, preliminary study: Bascom 181-309, 310; Geodicke 180-388; Hess 182-360; Lill 181-311; National Academy of Sciences 181-308

Mohorovičić discontinuity, depth: Nanda 180-242

nature: Harris 182-359; Kuno 181312

phase transition:MacDonald 182-358

Mongolia, earthquakes, macroseismic observations: Ninzhbadgar $182-101$

Montana, earthquakes, 1959: da Costa 182-84; Hopkins 182-85; Jackson 183-99; Nuttli 183-100;

Stewart 183-98

magnetic surveys, $10 \mathrm{~N}$ pluton: Zietz $183-488$

Moon, atlas, photographic: U. S. Air Force 181-76

atmosphere: Öpik 183-90

back side, photographed: Priroda 183-87

craters, incidence on far side: Beard 180-28

origin: Green 183-89

crustal structure: Bülow 183-51

maria: Lacroute 182-79

molten subcrust: Urey 180-27

origin: Havemann 183-91

seismic experiments: Press 183-88
Moon-Continued

surface activity, Alphonsus crater: Hédervári 182-80

cause: Windsor 180-26

surface features: Moore 182-77; Urey $182-78$

Morocco, age, beaches: Gigout 181-43

age, brannerite: Ledent 182-45

general listing: Choubert 183-25

earthquakes, 1960: Tillotson 181-93

\section{$\mathrm{N}$}

Netherlands, age, radiocarbon dates: Andersen 182-41

Neutron flux, measurement, U.S. S.R. : Shmonin 180-328

Nevada, crust, structure: Press 181305

crustal deformation, mechanism: Thompson 183-327

Nevada Test Site, gravity surveys: Diment 183-372

seismic surveys: Diment 183-372

New Brunswick, magnetic surveys, maps:Canada Geological Survey 183-493

New Guinea, isogonic map, epoch 1960. 5: Parkinson 180-255

oil exploration: Bureau of Mineral Resources, Geology and Geophysics 183-288

New Hampshire, age, submergence of coasts: Lyon 182-32

New Jersey, magnetic surveys, maps: Bromery 183-480; $183-481$

New Mexico, magnetic surveys: Andreasen 183-486

magnetic susceptibility, volcanic rocks: Wargo 181-381

New Zealand, age, peat: McKellar $183-44$

age, shells on old beaches: Schofield $183-45$

faulting, transcurrent movement: Lensen 180-201

geophysical surveys, Taupo Tarawera district: Modriniak 180170

geothermal energy: Mériel 182-541 gravity surveys: Robertson 182-327 magnetic surveys, Wairakei geothermal field: Studt 180-318

radioactivity, beach sands: Martin $183-511$

Newfoundland, age, igneous rocks: Fairbairn 181-41 
Nigeria, age, Precambrian: Schürmann 182-46

North America, age, glacial deposits: Rubin 182-34

isostatic adjustment: Sargent 183 339

North Carolina, coastal plain structure: Ferenczi 182-268

magnetic surveys: Johnson 183-530 radioactivity surveys: Johnson 183530

seismic survey: Bonini 181-469

Northwest Territories, age, Ellesmere Island: Blackadar 182-39

Norway, age, radiocarbon dates: $\mathrm{Ny}-$ dal 182-26

paleomagnetism, Late Precambrian: Harland 180-284

Nova Scotia, age, granites: Fairbairn $181-38,39$

age, submergence of coasts: Lyon $182-32$

vitrain: Tupper 181-40

magnetic surveys, maps:Canada Geological Survey 183-494, 496

seismic survey: Macpherson 183569

Nuclear explosions, fracturing: McKeown 183-200; Shoemaker 183202; Wilmarth 183-201

long period waves: Oliver 182-174; Rocard 181-176

maximum ground acceleration: Diment 183-199

PS converted waves :Schwind 183-204 seismic exploration: Bullen 181-468 seismic waves, Africa 1960: Rocard $181-175$

traveltimes: Kogan 183-206

strong motion measurements : Adams 182-175

Nucleogenesis:Eberhardt 183-71; Reynolds 183-66; Wasserburg 18369

Nutation, core to shell relationship: Fedorov 181-47

latitude observations: Fedorov 180$78,79,80$

mechanical characteristics of crust: Jeffreys 180-77

pole tides: Haubrich 180-73

theoretical values compared with observed values: Jeffreys 180-76

Oceans, origin, continental drift disputed: Lyustikh 181-244
Ohio, seismic surveys, ground-water problems: Warrick 181-471

Olivine-spinel transition: Ringwood $183-400$

Ontario, age, biotite: Aldrich 181-35 age, biotite and feldspar: Hurley 181-37

glacial deposits: de Vries 182-38 granite: Wetherill 182-37

syenite: Fairbairn 182-36

magnetic surveys: Steenland 182-424 maps: Canada Geological Survey $183-497$

seismic survey: Hodson 182-501

Oregon, magnetic surveys: Balsley $183-491$

Orogen, bilateral nature: Kraus 182260

conference, Würzburg, Germany: Harrison 182-266

Orogeny, causes: Bemmelen 183-329; Khain 183-319

depth, zone of low velocity: Scheidegger 181-247

evolution of process: Brock 182-263

Japan: Ehara 183-332

lateral thrust due to volume change: Wolfe 183-322

phases of process: Kraus 183-321 remanent magnetism: Kawai 182-265 spatial relation to continents: Gastil $183-324$

Overvoltage, historical summary: Brant 182-184

\section{$\mathbf{P}$}

Pacific Ocean, crust: Utsu 182-357 earthquakes, dislocation theory: Droste 181-114

geotectonics: Ehara 182-272

magnetic surveys: Agocs 180-313

microseisms: Tabulevich 183-503

radioactivity, carbon-14: Burling 180-329

rotation: Biq 182-273

Sala y Gomez ridge: Fisher 181-489 sea floor: Revelle 181-487

stress distribution: Balakina 180-47

Pakistan, magnetic field: Barta 181349

Paleomagnetism, Africa, Late Carboniferous to Late Triassic:

Nairn 180-289

Antarctica, Jurassic: Blundell 180292

Paleozoic and Mesozoic: Bull 181 392 
Paleomagnetism-Continued

Antarctica-continued

Precambrian:Nagata 180-291;182416

Canada, Precambrian: Du Bois 183462

chemical and pressure effects: Kawai 182-409

continental drift, evidence of: Collins on 183-459

England, Jurassic: Girdler 180-283

Late Paleozoic: Creer 180-281

England and Ireland, Cretaceous and Tertiary: Wilson 182-411

Europe, Paleozoic, Mesozoic, and Tertiary: Nairn 181-386

France, Cenozoic: Glangeaud 181387

Jurassic: Girdler 180-283

Miocene: Roche 182-412

Germany, Permian: Schmucher 180286

Iceland, Pliocene-Pleistocene: Rutten 183-463

Japan, Cretaceous and Tertiary: $\mathrm{Na}-$ gata 180-290, 181-391

magnetic stability of rocks: Frölich 181-380

magnetostrictive effects: Stott 182407

methods: Collinson 181-383; Creer 180-281; Everdingen 180-285

New Mexico, Clayton basalts: Baldwin 182-410

Norway, Late Precambrian: Harland 180-284

pole positions, catalog: Irving $181-$ 384

review of field: Cox 182-406

Scotland, Miocene: Khan 181-385

temporal subdivisions:Doell 183-457

U. S. S. R. , Cenozoic:Markhinin 181 390; Valiev 182-414

Devonian: Lin'kova 183-464

lava flows at Klyuchevskaya volcano: Bernshteyn 182-432

Pliocene and Pleistocene: Khramov 182-415; Pospelova 180-288; $182-413$

ratio of remanent magnetism to susceptibility: Pecherskiy 181389

Triassic: Makarova 181-388

United States, wind direction in Late Paleozoic: Opdyke 183-460

Papua, volcanic activity, Mount Lamington: Taylor 182-537

Paradox basin, geophysical surveys: Joesting 183-487
Pendulum apparatus, improved bronze type: Thompson 182-302

Zöllner and Lettau types: Jobert 182-304

Pennsylvania, age, uranium-bearing sandstone: Stern 183-16

magnetic surveys, maps: Bromery $183-478,479,480,481$

structure:Bromery 183-483; Zietz 183-482

Montana earthquake of 1959:Hopkins 182-85

Permafrost, classification: Pavlov 180-240

construction problems: Khaskind 180-239

U. S. S. R. : Grave 183-352; Zemtsov 180-238

Peru, crust, thickness: I. G. Y. Bulletin 182-350

Philippines, geophysical exploration, airborne: Rankin 181-222

magnetic survey: Agocs 180-313

Philippine Trench, isotopes, oxygen18: Dansgaard 183-406

Physical properties, underground nuclear test studies, Oak Spring formation: Keller 183-307

Planets, origin: Urey 181-75

Poland, age, radiocarbon dates: Tauber 182-20

geophysical surveys: Skorupa 183284

magnetic field base network, linked with Czechoslovakia:Bucha 182 380

Potassium analysis, thermal neutron irradiation: Goldstein 181-11

Potassium-40, energy spectrum: Kartashov 180-341

Prince Edward Island, magnetic surveys, maps: Canada Geological Surveys 183-495, 496

\section{$\mathrm{R}$}

Radioactivity, arsenic and antimony: Baranovskiy 180-332

Atlantic Ocean: Nelepo 183-517

atmosphere:E1 Nadi 183-518; Gorshkov 181-414; Hess 183-519; Kogan 182 -451; Lal 181-420; Lockhart 180-331; Malakhov 181-419; Peters 181-417; Rubin 182-29; Styro $182-452$

carbon-14: Burling 180-329

cosmic rays: Sastry $182-453$

distribution of radioactive elements : Davidson 181-406 
Radioactivity-Continued

earth's crust: Gorshkov 181-414

elements, emission at high temperature: Abdulgafarov 180-327

emanations, diffusion in porous media: Bulashevich 181-408

exploration, manual:Echagaray 182465

France, Mortagne granite massif: Roubault 183-509

gamma radiation: Kogan 180-325; Vincenz 181-412

Germany, Baden-Baden: Kirchheimer 183-510

Greenland, Skaergaard pluton: Hamilton 181-410

helium, emission at high temperature: Abdulgafarov 180-327

homogeneous geologic media: Kogan 181-421

instrumentation: Aglintsev 181-407; Jones 183-526; Kobayashi 183529; Oilweek 180-343; Voyutskiy 180-324

Italy, Biella granite-syenite: Fiorentini-Pontenza 182-445

Japan, feldspar and biotite: Hayase $182-446$

granites: Tsutsumi $182-447$

Ogamo and Ningyotoge mines: Hayase $182-448$

lead-210, decay constant: Eckelmann, 183-506

metallogenesis: Smyslov 182-331

neodymium: Karras 181-409

neutron radiation, surface of earth: Cherdyntsev 182-450

New Zealand, heavy minerals of beach sands: Martin 183-511

potassium-40: Kartashov 180-341

radon: Israël 180-330; Philip 181418; Tanner 181-413; Wilkening 183-514

rainwater: Rama 183-408

rocks, neutron emission: Gorshkov 183-507; Kashkarov 183-508

samarium: Karras 181-409

soils: Telfair 181-411

sphene: Hayase 180-326

uranium:Gokhshtein 182 -456; Kartashov 180-341

uranium minerals: Young 181-335 water, oil fields: Alekseyev 183-516

radon content: Jurain 183-515

Radioactivity exploration, adjunct to geological mapping:Guillou 183521
Radioactivity exploration - Continued airborne, Australia: Mumme 182466

capability reviewed: Moxham 181428

beryllium detector: Bowie 181-434; Derksen 183-528

emanation method: Tanner 183-520

gamma-ray spectrometer: Mero $183-527$

instrumentation, fallout effect:Wiles 183-524

measurement, field guide: Daly $181-$ 431

methods, discussion: Brownell 180344

French Africa: Bigotte 180-345

Uganda: Cawley 180-346

Union of South Africa: Nel 180-347 oil-gas fields: Alekseyev 183-525

radon migration:Timofeyev $181-432$ Spain, handbook: Serrano 181-430 thorium content, physical method for granite: Flanagan 183-522

U.S.S.R., petroleum: Laubenbakh 181-429

uranium, lead-isotope anomalies: Surazhskiy $182-467$

radon as guide: Tanner 183-523 Radioactivity logging, accelerated charged particles: Zaporozhets 180-335

bor on determination: Galuzo 181-425 calibration of instruments:Sulin 181 426

combination logger OKS-56: Pomerants 183-264

density log: Anderson 180-334; Edwards 180-339; Pickell 182-458

electrical logging, combined use: Becker 180-157

gamma method: Eife 180-342; Homilius 182-463; Itenberg 182-464; Oil in Canada 180-338

gamma and neutron-gamma methods: Zaporozhets 180-337

gamma radiation, distribution in rocks: Filippov 183-532

gamma spectrometry: Nedostup 180336; Zaporozhets 181-422

methods, review: Stick 182-459

U. S. S. R. :Sen'ko-Bulatnyy 180-340 neutron method: Blankov 181-424; Holgate 182-460; Stroud 180333; Tal'yanskiy 183-535; Temkin 182-462 
Radioactivity logging-Continued nuclear method, Appalachian Basin: MacFarlane 181-427

application: Nauta 182-461 porosity determination: Korzhev 183-533

potential of method: Mardock 182457

small-bore drill holes: Armstrong $183-534$

soil: Meigh 183-512

standardization of instruments: Zaporozhets 180-337

uranium exploration, radon: Tanner 183-523

water-oil interface:Blankov 181-423

Radioactivity surveys, Australia, Olary: Harris 180-360

Australia, Radium Hill: Hiern 182473; Mumme 182-474

France, Mortagne granite: Roubault 183-509

radon in natural waters: Jurain $183-515$

Japan, Chikuho: Nakai 180-359

Gifu: Nakai 180-356

Go River: Matsubara 182-469

Hiroshima and Takanawa: Sugiyama 180-351

Iwate: Horikawa 180-355

Kitakami: Iwasaki 180-348, 350

Kyushu: Iwasaki 180-353

Miyagi: Ujiie 180-354

Sakurago and Zomeki: Kinosaki $182-472$

Seto Inland Sea: Niino 183-513

Shikoku: Iwasaki 180-358

Shimane: Kaneko 180-352

Shinji and Tamatsukuri: Tokunaga $182-470$

Tottori: Horikawa 180-357

Uchitomi mine: Ukai 182-471

Yamaguchi: Iwasaki 180-349

Malaya: Agocs 180-314, 315

North Carolina: Johnson 183-530

Washington: Washington Division of Mines and Geology 183-531

Radon, air: Philip 181-418

concentration in drill holes: Tanner 181-413

soils: Israël 180-330

Red Sea graben, age of latest movements: Nesteroff 182-47; Voûte 183-340

Remanent magnetism, changes due to chemical and pressure changes: Kawai 182-409

deviation of vector of: Brodskaya 183-458
Remanent magnetism-Continued effect on anomalies:Girdler 183-469; Green 181-379

metamorphic rocks: Yasukawa 183452

movement of lava, effect of: Kruglyakova $182-408$

orogenesis: Kawai 182-265

reversals : Neumann $182-425$

Resistivity, diabase, proportional to Young's modulus: Yashchenko 183-275

pressure effect: Parkhomenko 182214

Rhode Island, age, Pennsylvanian: Hurley 181-26

Rift valleys, origin: Bishopp 182-262 Rock pressure, mines: Gzovskiy 183580

Rocks, physical properties: Kobranova 183-308

Rumania, earthquakes, magnitude recording: Iosif 181-107

geotectonics, recent deformation: Ez 181-246

gravity surveys: Airinei 181-283, 182-323; Botezatu 181-284, 286

Stoenescu 180-228, 229,181-285

magnetic field, absolute values: $\mathrm{Mi}$ lea 180-256

magnetic surveys: Airinei 180-311, 181-283; Stoenescu 180-228

\section{S}

Saskatchewan, age, radiocarbon dates: Kupsch 181-33

exploration, helium: Bateman 182223; Sawatzky 182-224

Satellites, geodetic observations: Thomas 182-254

gravity observations: Cook 182-287

Scintillation spectrometer, portable: Strauss 181-433

Scintillators, liquid:Cummins 182-455

Scotland, age, biotite and feldspar: Giletti 180-11

paleomagnetism, Miocene:Khan 181 385

Sea floor, relief: Menard 182-522

Sea level, changes due to deep convection currents: Gey1 182-274

Seamounts, Sala y Gomez ridge: Fisher 181-489

Seismic exploration, amplitude and frequency: O'Brien 182-485

blasting caps, static-resistant: Banville 183-564 
Seismic exploration-Continued

borehole geophones: Dennison 181 445; Zel'tsman 181-460

civil engineering: Suyama 183-555

continuous seismic profiler: Officer $181-454$

continuous seismic vibration: Crawford 180-379

diffraction problems, fault interpretation: Kunz 183-548

direct waves: Duda 183-181

engineering studies:Currier 182-495

exchange center: Bike 183-302

filters: Gol'tsman 180-385; Kalinina $181-439$

frequency analysis: Sengbush 180365

galvanometer coupled to electromagnetic seismograph pendulum: Matuzawa 180-387

gas-explosion method: Oilweek 181453

groups and phase velocities: Savarenskiy $182-481$

improvement of methods:Myers 180381

instruments, borehole shock producing device: Kovalev 183-567

calibration: Khokhlov 181-463

isonormals, conversion to isoverticals: Brož 181-440

lateral velocity variations: Olhovich $182-475$

magnetic recorder, time-sifter type: Frost 181-464

marine: Chujo 181-438; Kuroda 183566; Neprochnov 180-392, 182504

mass areal seismic surveying, U.S. S. R. : Zav'yalov 181-476

model study: Rao 182-493

motion at source:Kisslinger 183-536

ruclear explosions, utilization: Bullen 181-468

photomechanical frequency analysis : Sengbush 182-494

recording: Alekseyev 181-465; Graebner 180-363

reflection, air-shooting: Beránek 181-447; Rische 181-436

compensating filtering: $D^{\prime}$ Hoeraene 183-549

deep horizons: Zhuk 180-377

deformation of waves by sampling filter: Kawashima 182-490

geophone grouping: Shneyerson 181-441
Seismic exploration-Continued

reflection-continued

limestone structures: Richards $181-443$

line ups on records:Kametani 182 491

marine exploration: Zverev 182483

shot grouping: Ma 183-550, 551

spectra of waves: Berzon 180-370

traveltime function: Webster 183544

U. S. S. R. : Gurevich 181-455

vertical traveltime curve: Petkevich 183-552

refraction, boundary velocities:

Radzhabov 180-369

composite traveltime curve: Khramoy $182-488$

deep horizons: Deutsch 180-389; Veytsman 182-344

depth and relief of discontinuity; Radzhabov 182-477, 478

engineering problems: Hattori 183556

ghost reflections: Lindsey 180-361

methods: Adachi 182-492; Brown 180-386

nomograms: Meidav 183-553

remote control shooting: Vetterlein 181-448

salt domes: Musgrave 180-362

setting-up lines: Kurihara 181-449

surface shooting: Buffet 182-487

thickness of unconsolidated depos-

its: Brown 180-390

use of amplitudes:O'Brien 183-554

research, review for 1957-60: Faust $182-484$

rock-hardness indicator, tunnelplanning: Iz aki 183-557

scattering of waves: Riznichenko 180-372

seismic pulse frequency analysis: Moody 183-545

slide rule, calculation of $\mathrm{V}=\mathrm{V}_{\mathrm{O}}+\mathrm{KZ}$ : Bois $182-476$

soil, thickness determination: Ivanova $182-489$

stomper: Gregory 183-563; Kimbell $183-565$

Summarizer: Carissimo 183-562; Merlini 181-457; Oil in Canada 180-378; Savit 180-364

timing equipment: Riihimaa. 183-211 topographic effect on average velocity: Thralls 183-546 
Seismic exploration-Continued

trajectory and wave front diagrams: Olhovich 183-543

U.S.S. R., rivers of Western Siberia: Zverev 181-456

wave generation, small explosions: Kasahara 183-210

weak signals: Voyutskiy 183-547

Seismic focuses, regional structure, Africa Western Rift Valley: Berg 180-203

regional structure, U.S.S. R. : Petrushevskiy 180-46

Seismic stations, Jamaica, established by 1960: Robson 183-174

Seismic surveys, Alberta: Magrath $183-570$

Algeria: Bouchon 183-573

Antarctica: Sorokhtin 182-507

Appalachian region: Snow 181-470

Arctic: Crary 183-572

Atlantic Ocean, continental shelf: Ewing 183-583

Australia: Harris 180-395

Austria: Förtsch 181-474; Janoschek 183-574

California, continental shelf and slope: Moore 183-584

Canada, Northwest Territories and Arctic Ocean: Oilweek 183-571

Caribbean Sea: Goedicke 180-388; Officer 180-243

Czechoslovakia: Beránek 181-475, 182-320

Germany: Andres 182-503; Thomas 183-575

Greenland: Roethlisberger 182-502

Hawaii: Shor 183-396

Iceland: Båth 182-354

Indiana: Rudman 183-568

Japan: Kaneko 183-578

Louisiana: Conselman 181-467

marine, activity of 1957-60: Raitt $182-500$

crustal thickness: International Geophysical Year Bulletin 181473

Nevada Test Site: Diment 183-372

North America, east coast: Drake 180-202

North America and South America crustal structure: International Geophysical Year Bulletin 182350

North Carolina and South Carolina: Bonini 181-469

Ohio: Warrick 181-471

Ontario: Hobson 182-501

South Africa: Hales 182-352
Seismic surveys-Continued

Texas: Kuhn 181-472

U. S. S. R. , Caspian Sea: Neprochnov 182-504; Viktorov 181-477

central Asia: Ulomov 181-478

Cis-Caucasus: Pustil'nikov 180161

crustal studies: Gal'perin 182-356

Fergana: Pak 182-505; Rybin 180393

Grozny: Viktorov 180-391

Irkutsk: Pyatchin 182-506

Kalmyk A.S. S. R. : Ostrovskiy 180-163

Pamir-Alay zone: Kosminskaya 180-244

Uzbek S. S. R. : Godin 183-576

West Siberian Lowland: Tal'virskiy $182-499$

Seismic waves, amplitudes, nuclear explosions: Romney 180-96

angle of emergence: Gayskiy 180-58 attenuation: Iida 180-368

behavior at earth's core: Rykunov 181-132

behavior in loose ground: Savarenskiy 181-117

body waves, earthquake magnitude: Solov'yev 182-112

oscillatory character: Vaněk 183152

channel waves: Bäth $180-62,182-$ 129

conversion near shot point: Nanda 180-367

depth to basement: Bulin 183-153

dispersed wave trains: Brune 181118

dispersion, Love waves:Jobert 182 $130,183-157$

dispersion data: Levskin 182-133

dispersive surface waves: Yanovskaya 181-159

displacement: Pod"yapol'skiy 181 127

dynamic characteristics: Berzon 182-127; Kondorskaya 182-126

elliptically polarized:Gal 'perin 182 128

energy:Howell 182-172; Savarenskiy $182-115$

free oscillations of the earth: Gilbert 181-119

ground waves: Berg 180-97; Carder 180-95

head waves: Pod"yapol'skiy 181-437 
Seismic waves-Continued

hypocenter determinations: Asano 180-101

interference systems: Gol'tsman $182-479,480$

Lg: Herrin 181-137, 183-155; Utsu $182-357$

long period: Benioff 180-61

low-velocity layers: Gutenberg 183149

mantle: Vvedenskaya 181-320

model study, two-dimensional: Angona 181-173

Montana 1959, water-level in Pennsylvania: Hopkins 182-85

motion at source:Kisslinger 183-536 normal-mode and ray-optical interpretation: Tolstoy 181-120

nuclear explosions, high-altitude type: Pomeroy 183-203

origin time: Uyeda 180-263

traveltimes: Kogan 183-206

P-phase: Shurbet 182-125

P- and S-waves:Doyle 180-102;Kamitsuki 180-59, 60; Lehmann 182 363

PL phase, leaking modes: Oliver $183-154$

$P^{\prime} P^{\prime} P^{\prime} P^{\prime}$, analysis: Gutenberg 181122

polarization: Richter 181-139

propagation: Dix 180-86; Hervas

Burgos 182-155; Menzel 180-84;

1957-60: Press 182-149

Rayleigh, Atlantic earthquakes:

Crenn 182-131

dispersion: Porkka 183-399

energy: Kogan 182-132

particle amplitude profiles: Dorman 183-189

phase velocity: Nafe 183-156

shear-velocity distribution: Dorman 181-130

thickness of sediments: Shurbet 181-136

top of mantle: Enescu 181-125

vertical distribution of amplitudes: Kobayashi 181-138

Rayleigh and Love, earthquake mechanism: Aki 181-111

reflected, amplitude: Koefoed 181435

reflected refraction: Bortfeld 182486

refracted: Lossovskiy 181-126; Nikitin 180-371
Seismic waves-Continued

refracting boundaries, azimuthal station data: Glivenko 182-122

SP type: Glivenko 181-133

scattering, topographic irregularities: Gilbert 183-150

shadow zone of core:Gutenberg 181131

surface, Asiatic continent:Öcal 183158

Asio-African and Eurasian paths: Kovach 180-64

crustal structure: Sato 182-166 epicentral azimuths: Arkhangel'skaya 181-135

propagation: Takeuchi 181-134

two types: Perri 182-161

T -waves:Grinda 181-123; Khovanova 181-129; Wadati 183-151

transient: Ewing 181-124

triboelectric effect: Parkhomenko 181-169

underground explosions: Kogan 181 128

velocity, India: Quadir 181-479

Japan: Asano 180-100; Yoshikawa 180-394

modern marine sediments: Neprochnov 180-376

upper mantle: Gutenberg 180-56; Lehmann 180-57

volcanic activity: Kizawa 182-134, 183-159

Seismicity, Africa, West African Rift Valley: de Bremaecker 181-98

Asia, maps: Gorshkov 181-101

China, Gan'suy Corridor:Petrushevskiy 181-103

general characteristics: Mey 182 104

magnitude equation: Savarenskiy $182-103$

map: Shan-pan 182-102

Czechoslovakia: Kárník 182-92

Finland: Penttilä 183-115

gravity and magnetic anomalies:

Kurbanov 181-100

Iran: Kirillova 183-122

Italy: Malaroda 182-91

moon, feasibility study:Press 183-88

recent deformation: Kostenko 183-

124; Kurbanov 181-100

regionalization: Gzovskiy 182-89

Kats 182-90; Medvedev 183-114

Turkey: Kirillova 183-122 
Seismicity-Continued

U. S. S. R. , Armenian S. S. R. : Safaryan 183-133

atlas: Savarenskiy 181-99

Caucasus: Byus 183-117; Kirillova 183-121; Rastvorova 183-119

central Asia: Kostenko 183-124

Darial: Safaryan 183-116

Fergana: Kon'kov 183-126

Georgian S. S. R. :Rubinshteyn 183120

Kamchatka and Kuriles: Kondorskaya 181-102, 182-100

map: Medvedev 182-94

Moldavian S. S. R. : Sukhov 183-112

Primor'ye: Denisov 183-129

Sakhalin: Ferchev 183-131; Rudich 183-132

Siberia: Florensov 183-128

Tien Shan: Goryachev 182-99; Gzovskiy 182-96; Krestnikov 182-97; Fesenko 183-127

Trudniy Peninsula: Organova 183130

Turkmen S. S. R. : Bune 183-125; Puchkov 183-123

Seismograms, acceleration and displacement records:Walter 183161

azimuth, exchange and reflected waves: Glivenko 181-133

frequency characteristics: Arkhangel'skiy 181-442

interpretation, manual: Neumann 182-144

source functions: Aki 182-117

synthetic: Anstey 183-538; Baranov 183-540;Bois 183-539; Chauveau 182-163; Delaplanche 183-541; Dennison 183-537; Geophysical Prospecting 183-542; Wuenschel 180-380

time of shot: Aksenovich 181-462

trace-for-trace correction: Jensen $181-444$

Seismographs, accelerometer: Bryunelli 181-143

amplifiers: Mozzhenko 181-461

automatic: Fogel' 182-139

azimuth determination: Panasenko 183-162

band-rejection filters:Pomeroy 181 142

Benioff type: Melton 183-164

calibration: Karras 183-165; Kozlov 180-67

channel identity: Bereza 183-166
Seismographs-Continued

description of various types: Willmore 182-140

developments in 1957-60:Press 182 . 135

drum rotation, source of error: $\mathrm{Ka}$ mamoto 182-145

electromagnetic: Ingram 183-160 Miura 182-143

inexpensive types developed in Fin. land: Nurmia 183-169

long period: Benioff 181-140, 181 141; Gilman 183-167; Sutton 182. 141

pèndulum stabilizer: Fremd 183-17£ performance: Peterson 183-163

recorders: Hudson 183-168; Siivole 183-170

shielding: Suzuki 181-466

tape recorders:McLaughlin 180-383; Mori 180-384; Willis 180-382

telemetered seismic net, Tasmania Newstead 182-138

tornado records, tilt of crust: Kis slinger 180-321

U. S. S. R. , SVK-M and SGK-M models: Pasechnik 181-144

UZS-2 models: Obukhov 181-45\& United States, changes during 1957 60: Murphy $182-136$

Seismology, bibliography, Jan. -June 1958: Smith 180-32

electronic computers: Gilbert 182137

research 1957-60, United States: Woollard 182-83

United States, historical review:Byerly 181-85

Seismometers, rotational strain type: Watanabe 182-142

Seismoscope: Lyamzina 183-212; Obukhov 181-145

Self potential, sedimentary rocks: Campbell 181-199

sulfide ore bodies: Sato 180-114

Solar system, origin: Briggs 183-50; Egyed 182-58, 59; Woolfson $183-49$

Solids, physical properties, high pres sures: Davydov 181-153

Somaliland, age, radiocarbon dates: Barker 182-21

South Carolina, seismic survey: Bonini 181-469

South Orkney Islands, age, schists: Miller 183-20

Southern Rhodesia, age, radiocarbon dates: Barker 182-21 
Spain, age, radiocarbon dates: Crane $182-22$

earthquakes, effects on buildings: Bonelli Rubio 182-123

electrical surveys:Sell Cantalapiedra 183-247

magnetic surveys: Gaibar-Puertas 180-309

radioactivity exploration: Serrano $181-430$

Sphene, radioactivity: Hayase 180-326

Spherical harmonics, orogenic implications:Vening Meinesz 180-196

Spitsbergen, recent deformation:

Birkenmajer 183-343

Strain, crust: Gzovskiy 183-580

rocks: Riznichenko 183-193

Streaming potential, shales:Bernstein 180-140

Strength, comminution, role of stress waves: Rinehart 182-510

crust: Carey 182-514

granite: Matsushima 180-396; Kataoka 181-483

Griffith's theory: Clausing 182-512

ice:Butkovich 182-517, 518, 519;

Clark 182-516; Hilty 183-581; Jellinek 183-582; Kerr 182-520; Tabata 181-486, 182-521

marble:Kataoka 181-483; Mogi 180397

number of ruptures as a function of energy: Vinogradov 181-484

rocks: Handin 182-508

rock salt, limestone, and anhydrite: Brace 181-481

salt, cavities in: Serata 182-515

shear failure: Jaeger 181-482

shear waves: Ito 182-167

stress rate: Wuerker 182-509

time-dependent factor:Hardy 182 511

Young's modulus, determination at dam sites: Masuda 183-287

Stress, orientation of minerals: Jeffreys $182-513$

Stress waves, velocities: Ito $182-167$

Submarine canyons, Indian Ocean: Hayter 182-526

Submarine geology, Antarctic Ocean: Lisitsyn 180-400, 181-491

Arctic Ocean:International Geophysical Year Bulletin 180-399

Atlantic Ocean: Ewing 183-583

configuration of sea floor: Revelle $181-487$

general discussion: Zenkovich 180401
Submarine geology-Continued geophysical studies: Mason 182-349 Indian Ocean: Ewing 181-490

Kamchatka: Tikhonov 183-585

Mediterranean and Black Sea: Lacombe 182-525

microseisms: Malurkar 182-443

Pacific Ocean: Bromery 183-489; Emery 182-523

seamounts: Fisher 181-489

seismic surveys, activity of 1957-60: Raitt 182-500

Sudan, age, radiocarbon dates: Crane $182-22$

Sweden, age, Precambrian: Magnusson 183-29

age, radiocarbon dates:Östlund 18230

Switzerland, electrical surveys: Poldini 182-190

gravity surveys: Poldini 182-190

$\mathrm{T}$

Taiwan, place in circumpacific tectonics: Biq 183-331

Tanganyika, electrical surveys: King 180-308

magnetic surveys: King 180-308

Tasmania, electrical surveys: Davidson 180-319

magnetic surveys:Davidson 180-319

telemetered seismic net: Newstead $182-138$

Tektites, age: Gentner 182-75; Pinson 181-73

aluminum-26: Anders 182-66

australites: Baker 183-83

composition, relation to granite:

Whitten 183-80

composition and magnetic properties: Friedman 183-81

germanium content: Cohen 183-84

indochinites: Vorob'yev 181-74

major element relationships:Cherry 183-78

moldavites: Vorob'yev 183-86

nickel and iron abundances: Ehmann 183-82

origin: Baker 181-71; Hawkins 181-

72, 182-76; Lovering 183-77;

Urey 183-79; Vorob'yev 183-85

Telluric currents. See Earth currents

Temperature, mantle and core: Gilvarry 181-296

Tennessee, electrical logging, $z$ inc deposits: Keller 183-268

Texas, seismic surveys:Kuhn 181-472 
Theodolite, electrodynamic: Lucke 181-342

magnetic: Reusche 181-343

Thermal activity, U. S. S. R.: Nekhoroshev $182-542$

Thermal springs, Fiji Islands: Healy $183-600$

geysers: Golovina 183-596

Japan:Ando 182-545; Nakamura 181$501,182-544$

U. S. S. R. : Markhinin 182-543; Tolstikhin 183-599

Thermoluminescence, age determination, granite: Komovskiy 181-20

Antarctic rocks, past climate: Zeller 182-57

limestones, Greenland: Zeller 18218

Thermometers, borehole: Aksel'rod 181-301

Transcurrent faulting, volcanic activity: Pakiser 181-240

Tsunami, causes: Popov 180-49; Savarenskiy 180-48

prediction, U.S.S. R.:Solov'yev 18050

T-waves, caused by:Khovanova 181129

Turkey, age, radiocarbon dates: Barker 182-21

electrical surveys:Dizioğlu 182-189

seismicity, map: Kirillova 183-122

\section{$\mathrm{U}$}

Uganda, radioactivity exploration:

Cawley 180-346

Ultrasonic velocities, granular rocks: Balakrishna 182-170

Union of South Africa, crust, structure: Gane 182-353; Hales 182352

gravity, observations:Gough 181-272

gravity surveys: Weiss 180-307;

Hales 182-352

isostasy: Hales 180-227

magnetic field: Heirtzler 183-422

magnetic surveys: Weiss 180-307

meteorites, Barkly West: Groeneveld 182-74

radioactivity exploration: Nel 180347

seismic survey: Hales 182-352

U.S.S.R., age, Aldan shield: Sudovikov 182-50

age, Bavly series: Timergazin 18148
U. S. S. R. -Continued

age, Belomorye complex: Shurkin $182-49$

charnockite, Ukraine: Komlev 1806

glauconite: Polevaya 183-33; Rubinshteyn 181-2

granite: Atrashenok 183-40; Komlev 180-5, 6, 183-38; Rubinshteyn 181-2; Studenikova 1804

igneous rocks: Bykovskaya 182-51; Firsov 182-53; Polevaya 182-52 Karelia: Kratts 182-48

micas: Rubinshteyn 181-45

pegmatites: Zhirova 181-49

radiocarbon dates: Stuiver 182-23

syenite: Rubinshteyn 181-2

terrestrial deposits:Krylov 181-50

time scale: Semenenko 182-3

Ukraine SSR: Komlev 183-36; Polevaya 181-46; Semenenko 18334; Vinogradov 181-47, 183-35, 37

Urals and Pri-Ural areas:Ovchinnikov 183-39

water:Kortsenshteyn 180-22; Mavritskiy 180-21

wood and peat: Vinogradov 181-17

age determinations, 1958 status: Starik 181-4

crust, structure: Godin 183-576; Ulomov 181-478; Veytsman 182344

thickness:Gal'perin 182-356; Moiseyenko 183-398

earth currents, diurnal disturbance: Mishin 181-83

exploration: Vladimirov 181-192; Zagarmistr 181-193

earthquakes, Azerbaijan: Sultanova 182-95

Baku, 1958: Gorin 181-96; Kuznetsov 181-95

epicenters: Pachadzhanova 180-66

Gobi, 1957: Pasechnik 180-44; Solonenko 183-113

Kamchatka:Benioff 180-61;Stauder 183-139

Kurile-Kamchatka zone: Kondorskaya 182-93

magnitude and intensity: Savarenskiy 182-114

magnitude and energy: Salov'yev $182-112$

North Caucasus: Nikitin 182-118 
U. S. S. R. - Continued

earthquakes-continued

seismicity atlas: Savarenskiy 181 99

Tadzhik S. S. R. : Gayskiy 180-38; Gubin 180-41; Kon'kov 180-42; Leonov 182-87; Semenov 180-40

Tien Shan: Vvedenskaya 182-98

Ulugchat 1955: Leonov 180-43

electrical exploration: Krayev 183248

electrical logging surveys: Chernyshev 180-144; Dolitskiy 180-145; Itenberg 183-271; Umantsev $182-210$

electrical surveys, ground water: Shayins'kyy 182-193

electromagnetic exploration: Tikhonov 181-197

fault plane solutions: Scheidegger $182-121$

geodetic datum: Izotov 180-193

geophysical exploration, 1959-65: Geologiya Nefti 181-228

combination of methods: Burtmar 181-219

cost analysis: Kozlov 181-217

evaluation: Brod 181-216

gold: Safronov 182-243

methods: Andreyev 182-242

organization: Kozlenko 181-218

geophysical research, evaluation:

Khitarov 181-229

geophysical surveys, Astrakhan:

Rakitov 180-164

Caspian Sea: Mustafayev 183-285

Dnieper-Donets depression: Andreyeva 181-221

Georgian S. S. R. : Balavadze 181307

Kuybyshev: Yelanskiy 182-228

Siberia: Savinskiy 181-227

summary for 1955-58: Godin 182241

Turkmen A. S. S. R.: Kasatkin 181230

Volga area: Godin 181-215; Kozlenko 181-220

geophysical well logging: Buryakovskiy 180-165; Dakhnov 182-206 geothermal gradient, Armenian S. S. R. : Ananyan 183-391

West Siberian Lowland: Koshlyak 182-336; Ryabukhin 182-337

geothermal zoning, West Siberian Lowland: Mavritskiy 181-299

gravity exploration: Oganizyan 181 291; Uspenskiy 182-298
U. S. S. R. - Continued

gravity surveys, Armenia: Oganisyan 181-290, 182-324

Bashkir and Tatar A. S. S. R.: Volodarskiy 180-231

Caspian area and Ustyurt: Nevolin $181-288$

Emba region: Kotlyarevskiy 181 289

Kalmyk A. S. S. R. : Ostrovskiy 180163

Kuban River and Caspian Sea areas: Dzhafarov 183-380

Minusin trough: Tarkov 180-230

proposed network: Grushinskiy 181-287

Rudnyy Altay and Kalba: Gorzhevskiy 181-292

Siberian platform: Vasil'yev 180232

Tatar A. S. S. R.: Boronin 183-381; Salikhov 183-382

West Siberian Lowland: Agul'nuk 182-430; Zakashanskiy 182-431

iron deposits, magnetic properties: Arutyunyan 183-456

magnetic surveys, Armenia: Arutyunyan 182-427

Chernigov magnetic anomaly: Andreyeva 183-500

Klyuchevskaya volcano:Bernshteyn $182-432$

Kursk magnetic anomaly: Lapina 183-471; Shneyerson 181-400

Tatar A. S. S. R. : Stepanov 182-428

Vyatka and Kama River basins:Yarosh 182-501

West Siberian Lowland: Agul'nuk 182-430;Zakashanskiy 182-431; Pudovkin 182-429

meteorites, Manych chondrite: Gnilovskoy 181-68

microseisms, Caspian Sea: Tabulevich 180-322

Far Eastern Region: Bukhteyev 181-404

storm location: Monakhov 182-440

neutron flux, measurement: Shmonin 180-328

paleomagnetism, Cenozoic: Markhinin 181-390; Valiev 182-414

Devonian: Lin'kova 182-464

Pliocene-Pleistocene: Khramov 182-415; Pospelova 180-288, $182-413$

ratio of remanent magnetism to susceptibility: Pecherskiy 181 389 
U.S.S. R. -Continued

paleomagnetism, Triassic:

Makarova 181-388

permafrost:Grave 183-352; Zemtsov 180-238

radioactivity logging: Itenberg $182-$ 464; Sen'ko-Bulatnyy $180-340$

radioactivity surveying, petroleum: Laubenbakh 181-429

recent deformation: Meshcheryakov $182-271$

reference ellipsoid: Izotov 180-193 resistivity logging: Grechukhin $180-$ 146

seismic exploration, mass areal surveying: Zav'yalov 181-476

reflected waves: Gurevich 181-455 rivers of Western Siberia: Zverev $181-456$

seismic focuses:Petrushevskiy 18046

seismic surveys, Caspian Sea: Neprochnov 182-504; Viktorov $181-477$

central Asia: Ulomov 181-478

Cis-Caucasus: Pustil'nikov 180161

crustal studies: Gal'perin 182-356

Grozny: Viktorov 180-391

Irkutsk: Pyatchin 182-506

Kalmyk A. S. S. R.:Ostrovskiy 180163

Pitnyak: Sungurov 180-162

Uzbek S. S. R. : Godin 183-576

West Siberian Lowland:Tal'virskiy $182-499$

seismic waves, dynamic characteristics: Kondorskaya 182-126

seismicity,' Armenian S. S. R. : Safaryan 183-133

Caucasus: Byus 183-117; Kirillova 183-121; Rastvorova 183-119

central Asia: Kostenko 183-124

Darial: Safaryan 183-116

Fergana: Kon'kov 183-126; Pak 182-505; Rybin 180-393

Georgian S. S. R. : Rubinshteyn 183120

Kuril-Kamchatka: Kondorskaya 181-102

Moldavian S. S. R.: Sukhov 183-112

Primor'ye: Denisov 183-129

regionalization: Medvedev 182-94

Sakhalin: Ferchev 183-131; Rudich 183-132

Siberia: Florensov 183-128
U. S. S. R. -Continued

seismicity-continued

Tien Shan:Fesenko 183-127; Goryachev 182-99; Gzovskiy 182-96; Krestnikov 182-97

Trudniy Peninsula: Organova 183130

Turkmen S.S. R. : Bune 183-125; Puchkov 183-123

tectonic movements, relation to structure: Kukhtikova 180-39

thermal activity, Kambal'nyy Range: Nekhoroshev 182-542

thermal springs, Kamchatka: Tolstikhin 183-599

Kunashiri Island: Markhinin 182543

tsunami, prediction: Solov'yev 18050

volcanic eruptions, Bezymyanny: Gorshkov 180-406

Koryakskiy volcano:Gushenko 182 533; Sirin 182-532

volcanoes, description: Svyatlovskiy 180-403

United States, age, Early Man: Mason 182-33

age, radiocarbon dates:Bray $182-27$; Crane 182-22; Hubbs 182-31; Stuiver 182-23

earthquakes, 1957: Brazee 180-33

1958: Brazee 183-97

exploration drilling, 1959: Blanpied 183-277

geodesy, activity of 1957-59: U.S. Coast and Geodetic Survey 182 244

magnetic surveys, 1957-60: Nelson $182-378$

microseisms, research 1957-60: Carder 182-435

paleomagnetism, Late Paleozoic: Opdyke 183-460

seismic wave propagation, research 1957-60: Press 182-149

seismographs, changes during 1957 60: Murphy $182-136$

seismology, historical review: Byerly 181-85

research 1957-60:Woollard 182-83

Utah, geophysical logging: Millard 181-224 
Van Allen radiation belt, geomagnetic variations: Akasofu 181-354; Dessler 181-337

Velocity logging. See acoustic logging Venezuela, geophysical well logging: Haeberle 182-212

gravity surveys: Hospers 182-311, $183-377$

Volcanic activity, Australia: Sprigg 181-500

Azores: Mendonça Dias 180-402

Columbia: Ramirez 180-404

earthquakes:Kizawa 183-159; Minakami 182-538, 540

eastern Pacific Ocean basin: Richards 182-531

effect of water: Yamasaki 180-409 heat source, gases: Seino 180-412 Iceland: Vlodavets 181-497

Japan: Sato 181-499; Yoshikawa 180408

occurrence in belts: Ma 182-530

prediction: Mizutani 180-411; Minakami 182-539; Suwa 180-410; Vlodavets 181-493

transcurrent faulting: Pakiser 181240

volcanic necks: McBirney 181-492

world review: Trevisan 181-225

Volcanism, mechanism: Pakiser 183586

Volcanoes, Azores: Tazieff 181-496

Central America: Bullard 183-593 Chile, maars: Illies 180-405; Miller 183-594

Guatemala: Williams 183-589

Hawaii, growth: Eaton 183-588

Kilauea: Bellair 181-494; Friedman 182-527; Simpich 181495; Shale Shaker 183-592

phreatomagmatic explosions: Fraser 183-587

Iceland:Thorarinsson 182 -529; Vlodavets $181-497$

Italy: Cavallero 181-498

Japan: Matsumoto 182-535; Namba 182-534; Taneda 180-407; Morimoto 183-595; Sawata 183-591; Sekiya $182-536$

Papua: Taylor 182-537

U.S. S.R.: Gorshkov 180-406; Gushenko 182-533; Karapetyan 183-590; Sirin 182-532;

Svyatlovskiy 180-403
Volcanology, review: Rittmann 182528

W

Washington, crustal structure: Neumann 182-357

geophysical surveys:Brown 183-309

magnetic and radioactivity surveys: Washington Division Mines and Geology 183-531

West Indies, Dominica, unidentified tremors: Robson 183-110

Whistlers, geomagnetic disturbances, relationship between: Kimpara 181-369

Wisconsin, crust, thickness: I. G. Y. Bulletin 182-350

Wyoming, geophysical surveys:Peters $182-219$

\section{ERRATA IN BULLETIN 1116}

180-94, p. 30 (Bull. 1116-A)

On lines 5 and 6, read "meters" for "miles"

180-290, p. 85 (Bull. 1116-A)

Second line should read

"Kobayashi, Kazuo, and Kuno, Hisashi. "

180-291, p. 86 (Bull. 1116-A)

First line should read

"Nagata, T[akesi], and

Shimizu, Y[oshio]."

181-275, p. 208 (Bull. 1116-B)

First line should read

"Woollard, G[eorge] P[rior],

Ostenso, N[ed] A., Thiel, $\mathrm{E}$ [dward], and Bonini, W[illiam] E." 


